\title{
Elemental resolved magnetism of Gadoliniumnitride layers and GdN/Fe multilayers
}

\author{
Dissertation \\ zur Erlangung des Doktorgrades der \\ Mathematisch-Naturwissenschaftlichen Fakultäten der \\ Georg-August-Universität zu Göttingen
}

Vorgelegt von

Frank Leuenberger

aus Bern/Schweiz

Göttingen 2004 
D7

Referent:

Prof. Dr. W. Felsch

Korreferent:

PD Dr. P. Schaaf

Tag der mündlichen Prüfung: 
It is fortunate that physicists suffer the genetic defect of optimism.

Leon M. Lederman 



\section{Contents}

$\begin{array}{lll}1 & \text { Introduction } & 7\end{array}$

2 Experimental Methods $\quad 9$

2.1 Sample preparation . . . . . . . . . . . . . . . . . . 9

2.2 X-Ray diffraction . . . . . . . . . . . . . . . . . . . . . 11

2.3 Chemical Analysis . . . . . . . . . . . . . . . . 13

2.3.1 Nuclear Reaction Analysis . . . . . . . . . . . 13

2.3.2 X-Ray Photoemission Spectroscopy . . . . . . . . 13

2.4 Magnetometry . . . . . . . . . . . . . . . . . 15

3 X-Ray Magnetic Circular Dichroism 17

3.1 X-ray absorption . . . . . . . . . . . . . . . 17

3.2 XMCD Spectroscopy . . . . . . . . . . . . . . . . . . . . 18

3.3 Beamline description . . . . . . . . . . . . . . . . . 21

4 Rare Earth Pnictides $\quad 24$

4.1 Rare Earth Magnetic Semiconductors . . . . . . . . . . . . . 24

4.2 Electronic structure of the Rare Earth Pnictides . . . . . . . . 26

4.3 Preparation and structure of GdN . . . . . . . . . . . . . . . 28

4.3 .1 Chemical Analysis . . . . . . . . . . . . . 30

4.4 Magnetic and electronic properties of GdN . . . . . . . . . . 33

4.5 XMCD study of $\mathrm{GdN} \ldots \ldots \ldots$. . . . . . . . . . . 40

4.5.1 XMCD of the Gd L-edges . . . . . . . . . . . 40

4.5.2 Nitrogen K-edge dichroism . . . . . . . . . . . . . 46

4.6 Summary . . . . . . . . . . . . . . . . . . . . . 52 
$5 \mathrm{GdN} / \mathrm{Fe}$ multilayers $\quad 53$

5.1 Magnetism in RE transition metal intermetallics and multilayers 53

5.2 Sample preparation and structure . . . . . . . . . . . . . 56

5.3 Macroscopic Magnetization . . . . . . . . . . . . . . . . 60

5.4 Field dependence . . . . . . . . . . . . . . . . . . . . . . . . . . 69

5.4 .1 Dichroism of the Gd L-edges . . . . . . . . . . . . . 71

5.4 .2 Dichroism of the Fe L-edges . . . . . . . . . . . 76

5.4.3 Dichroism of the Gd M-edges . . . . . . . . . . 78

5.5 Elemental resolved Hysteresis . . . . . . . . . . . . . . . . 81

5.6 Summary . . . . . . . . . . . . . . . . . 85

6 Summary $\quad 86$ 


\section{Chapter 1}

\section{Introduction}

The miniaturization in microelectronics and the need for functional compounds has brought up many new tasks in material processing. Due to the constantly shrinking length scale a deeper understanding of the structure and the interfaces of the applied materials is indispensable. The field of 'spintronic' involves the spin of the electrons as a new parameter and opens up a new class of electronics. The magnetic random access memory (MRAM) is an example of such a new device. The advantage of MRAM is the fact that the magnetization direction of ferromagnetic TMR (tunnel magneto resistance) devices is used for storing information. This is an intrinsic property of the ferromagnetic films used and the functionality of the TMR cell is achieved with a simple multilayer stack. The lateral dimensions of the cell are therefore not an elementary part of the design and the size of the cell can be smaller than in conventional logic designs. Since the magnetization direction is persistent the devices are nonvolatile. A combination of conventional microelectronic based on semiconductors and spintronic is a promising field of research. But the implementation of metallic magnetic films into semiconductor technique is problematic since the polarization of the current provided by a metallic film is not easily injected into a semiconductor due to the large resistance mismatch of the materials $[1,2,3]$. A possible solution are ferromagnetic semiconductors as spin-filter devices. The effort in this direction has brought up new research on diluted magnetic semiconductors (DMS) like $\mathrm{Ga}_{x} \mathrm{Mn}_{1-x} \mathrm{~N}$ [4] or $\mathrm{Ga}_{x} \mathrm{Gd}_{1-x} \mathrm{~N}$ [5], but has also recovered the interest in ferromagnetic semi- 
conductors like $\mathrm{EuO}$ and $\mathrm{GdN}$, that were subject of rich investigations during the 70's. Also multilayer systems have gained a tremendous potential during the last 20 years since they offer new possibilities for functional devices especially in modern microelectronics. The field of application is widespread and covers, for instance, sensors in the automotive industries, magnetic recording media and new microelectronic devices like spinvalves. Due to the ongoing miniaturization in microelectronics the control over the 'feature size' of complex devices is one of the strongheaded problems nowadays. In multilayer systems the feature size is the layer thickness which is manageable down to atomic resolutions today in contrast to the lateral resolution, which is much harder to handle. A great example is the GMR head of a modern Hard Disk Drive (HDD). The fundamental functionality of the head is achieved by a 'simple' multilayer of $\mathrm{NiFe} / \mathrm{Cu} / \mathrm{Co}$ independently of its lateral size. Only the combination of the materials, the layer thicknesses and the interface contain the necessary physics. One can easily imagine that the role of the interface in multilayer systems is of accentuated interest and the understanding of the interplay of the materials at the interface is a very important point. 


\section{Chapter 2}

\section{Experimental Methods}

\subsection{Sample preparation}

The preparation of all thin film samples in this work was done in an Ultra High Vacuum (UHV) System. The system is divided into four sections (Fig. 2.1):

1. The preparation chamber for the Rare Earth nitrides and multilayers.

2. The preparation chamber for oxides (not used here).

3. An analysis chamber with a XPS spectrometer, a RHEED system and an Ar sputter gun (ion etching).

4. A transfer system with a load lock

All samples were prepared by ion-beam sputtering with a Kaufman type ion source with Ar as sputter gas. High purity targets (rare earth 99.9\%, transition metals $99.99 \%$ ) of $\mathrm{Gd}, \mathrm{Fe}, \mathrm{Cr}$ and $\mathrm{Al}$ are mouted on a rotateable target cube to provide an easy selection of the target material and to enable an automated sputter process of multilayer systems. The base pressure in the chamber is better than $5 \cdot 10^{-10}$ torr. The partial pressure of reactive gases like $\mathrm{O}_{2}$ or $\mathrm{CO}$ are monitored with a quadrupol mass spectrometer and are below $10^{-10}$ torr. All sputter gases $\left(\mathrm{Ar}, \mathrm{N}_{2}\right)$ have a $6 \mathrm{~N}$ purity $(99.9999 \%)$. The transition metals are sputtered with a $800 \mathrm{eV} \mathrm{Ar}^{+}$ion beam. The Ar pressure during the sputter process in the chamber is about $5 \cdot 10^{-5}$ torr and up to 


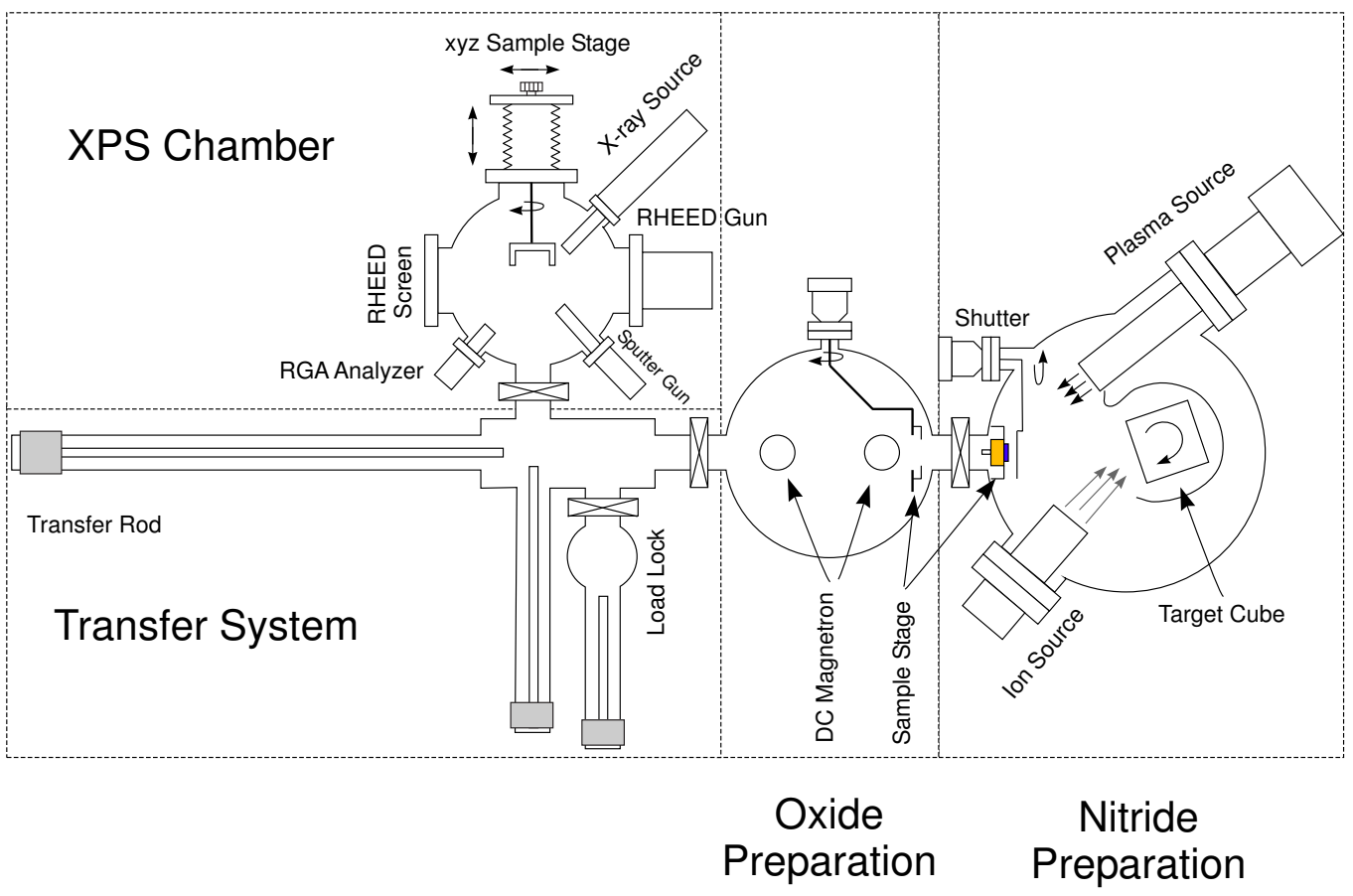

Figure 2.1: UHV Preparation System with in situ XPS and RHEED analysis. The oxides and the nitrides are prepared in different chambers. Up to six different sputter targets can be used at the same time.

two orders of magnitude lower than with conventional magnetron sputtering. The Gadolinium nitride films were prepared by a reactive sputter process at a $\mathrm{N}_{2}$ background pressure of $2-3 \cdot 10^{-5}$. To improve the reaction of the nitrogen with the growing film an ECR plasma source facing the sample is used to dissociate the $\mathrm{N}_{2}$ molecule into radicals and to generate a nitrogen beam with thermal energy. The sample stage can be heated up to $700^{\circ} \mathrm{C}$ and a shutter masks the substrate when the desired film thickness is reached. The thickness of the growing film is monitored by a quartz-crystal thickness monitor. With this method the film thickness can be controlled with an accuracy of $1 \AA$. The shutter and the target cube are motor controlled and the film thickness is monitored by a personal computer that opens/closes the shutter and rotates the target cube making a fully automated preparation of multilayer systems with a large number of bilayers possible. The substrates used in this work are Si (100), Kapton ${ }^{\top M}$ and Mylar ${ }^{\top M}$. For structure and macroscopic magnetic measurements the samples were prepared on Si. The x-ray absorption and x- 
ray magnetic circular dichoism (XMCD) spectra (see chapter 3) were mainly measured in the transmission geometry, so a Si substrate is to thick. In this case the samples were prepared on Mylar or Kapton depending on the energy range of the x-ray experiment. In the low energy range $(h \nu<1.5 \mathrm{keV})$ the samples were prepared on $1.5 \mu \mathrm{m}$ thin mylar foils. The mylar foil was first coated with an $\mathrm{Al}$ film on the backside $(\approx 500 \AA)$ and then glued with the coated side onto small copper frames to achieve a better thermal conductance of the foil. The frames were mounted onto the sample holder and a $100 \AA \mathrm{Al}$ film was sputtered on the foils as a buffer. Then the samples were grown onto the buffer at room temperature. The thickness of the samples is limited to around $200-300 \AA$ for the x-ray absorption experiments at low energy $(h \nu<1.5 \mathrm{eV})$. The thicker Kapton foils $(7.5 \mu \mathrm{m})$ were used for absorption experiments in the higher energy range $(h \nu 7-8 \mathrm{keV})$. These foils are much easier to handle and did not need a frame. The film thicknesses on the Kapton foils are typically up to $4000 \AA$.

The load lock and the transfer system provide a complete in situ UHV handling of the samples including the analysis with RHEED or XPS/Auger of the grown films (or multilayer) or ion etching of the surface. The sample stages permit heating up to $700^{\circ} \mathrm{C}$ in each chamber at UHV conditions or in an atmosphere of $\mathrm{N}_{2}$ or $\mathrm{O}_{2}$ (excluding transfer and load lock).

\section{$2.2 \quad$ X-Ray diffraction}

Since many properties of a solid depend strongly on the crystal structure and the lattice spacing, one needs experimental access to the microscopic structures of a solid on a sub-Angstrøm length scale. A widely used and common method to determine the crystal properties of a solid is X-Ray Diffraction (XRD).

The structure of the prepared thin films and multilayers were determined with a Siemens D5000 two-axis diffractometer with $\mathrm{Cu}-\mathrm{K}_{\alpha}$ radiation. All diffraction patterns were recorded in $\Theta-2 \Theta$ Bragg-Bretano geometry. In this geometry the scattering vector $\vec{q}=\vec{k}^{\prime}-\vec{k}$ is always perpendicular to the surface and one obtains information about the structure parallel to the surface normal. Depending on the angle $\Theta$ the method probes either the 


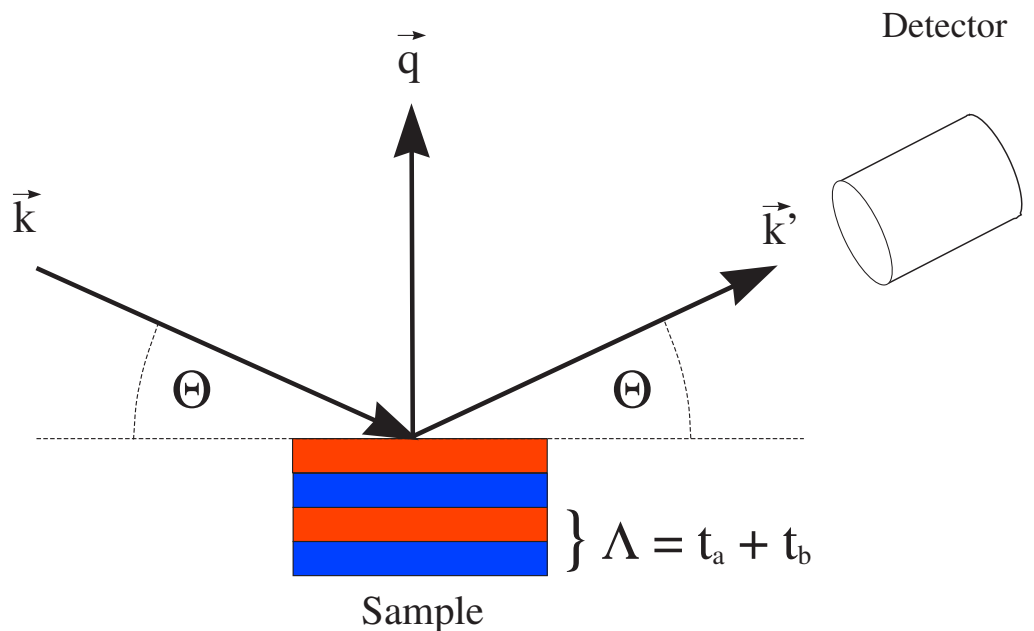

Figure 2.2: Schematic view of the $\Theta / 2 \Theta$ Bragg-Bretano geometry. The scattering vector $\vec{q}=\vec{k}^{\prime}-\vec{k}$ is parallel to the surface normal.

electron density of the chemical structure $\Lambda\left(0.5^{\circ}<\Theta<10^{\circ}\right)$ or the crystal structure of the atomic planes $\left(\Theta>10^{\circ}\right)$. From Bragg's law

$$
n \lambda=2 d \sin \Theta
$$

and the position of the reflections the lattice parameter of the crystal can be calculated. The crystallite size in growth direction $\left(D_{h k l}\right)$ is given by the Scherrer formula

$$
D_{h k l}=\frac{K \lambda}{B \cos \Theta}
$$

where $B$ is the FWHM of the peak at position $2 \Theta$ and $\mathrm{K}$ is the form factor, which is usually set to 0.9 for spherical crystallites [6]. In the small angle range the intensity can be calculated from the optical theory $[7,8]$. The optical density is transfered into a scattering potential that reflects the chemical periodicity of the sample. From the resulting reflectivity oscillations in the diffraction pattern the film thickness, or in case of multilayers, the chemical periodicity $\Lambda$ can be obtained. From multilayers with a large number of repetitions one observes narrow bragg peaks, whose intensity decreases for higher angles. The decrease of the intensity is very sensitive to the interface roughness and a multilayer with sharp interfaces produces multiple clearly observable Bragg peaks. The simulation of the oscillations can be done nu- 
merically $[9,10]$ and one can determine the film (layer) thickness and the rms roughness of the sample.

\subsection{Chemical Analysis}

\subsubsection{Nuclear Reaction Analysis}

The resonant nuclear reaction analysis (RNRA) is a suitable method to obtain the nitrogen concentration profile of a sample. It is based on a nuclear reaction of a proton beam with the nitrogen ${ }^{15} \mathrm{~N}$ isotope $\left({ }^{15} \mathrm{~N}(p, \gamma \alpha){ }^{12} \mathrm{C}\right)$ at a resonant energy of $E_{r}=898 \mathrm{keV}$. The reaction product is an excited ${ }^{12} \mathrm{C}$ nucleus and an $\alpha$ particle. The relaxation into the ground state of the ${ }^{12} \mathrm{C}$ produces a $\gamma$-quantum with a energy of $4.43 \mathrm{MeV}$, which is detected by a scintillation detector. After calibration with a sample with known concentration the counting rate is an absolute measure of the nitrogen concentration. The resonant character of the reaction enables the possibility to measure concentration profiles. Due to the deceleration of the proton beam in the sample the energy $E_{r}$ is reached at the depth

$$
d=\frac{\left(E-E_{r}\right)}{d E / d x}
$$

where $E$ is a beam energy $E>E_{r}$ and $d E / d x$ is the stopping power of the probed material. The resolution of the profile is limited by the energy sharpness of the beam and the energy broadening due to scattering in the sample to a few nanometers. The accuracy of the method is about 5\%. A more detailed description can be foud in [11].

\subsubsection{X-Ray Photoemission Spectroscopy}

With X-ray Photoemission Spectroscopy (XPS) the chemical composition of a specimen can be determined by measuring the kinetic energy of the emitted electrons. By irradiating the sample with an electromagnetic wave of energy $h \nu$, electrons with a binding energy $E_{B}<h \nu$ are excited and can leave the sample (photo effect). The kinetic energy of the electrons leaving the sample 


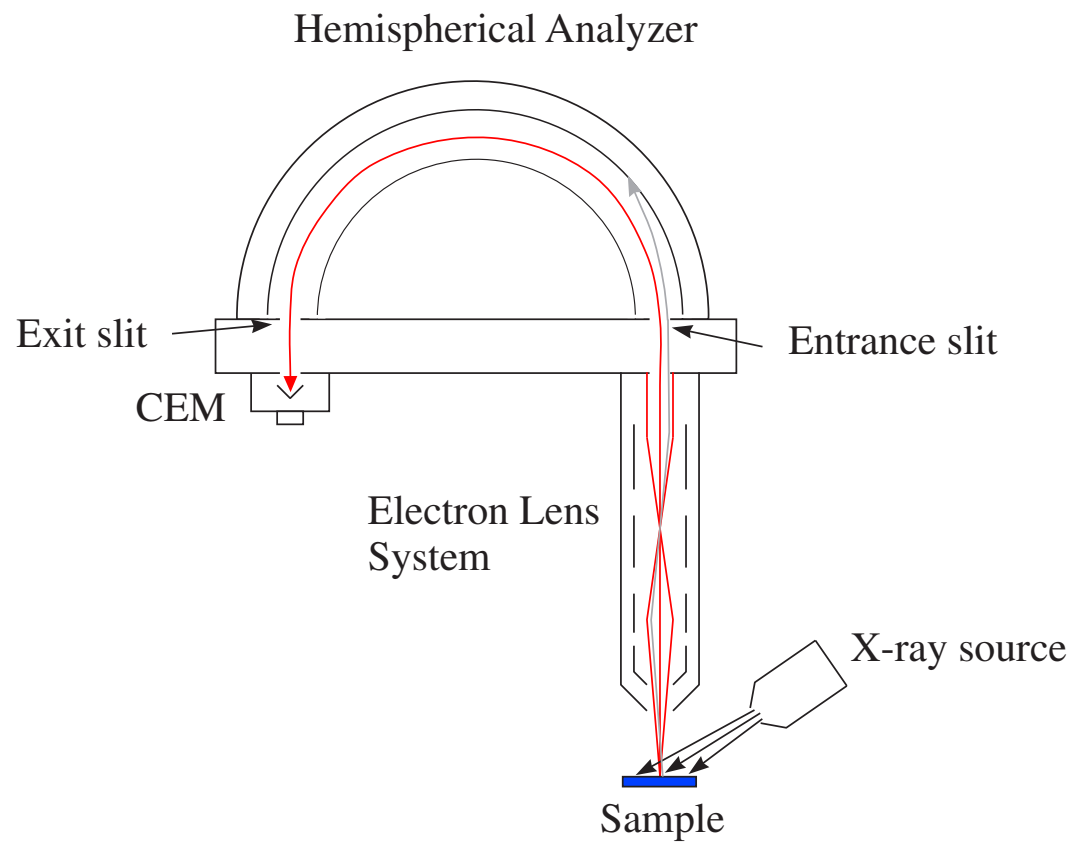

Figure 2.3: Schematic setup of a XPS spectrometer with x-ray source, electron lens system and a hemispherical analyzer with a channel electron multiplier (CEM). The red trajectory belongs to an emitted electron matching the energy condition of the analyzer. The kinetic energy of the electron on the gray trajectory is to high.

is given by

$$
E_{K i n}=h \nu-E_{B}-\phi
$$

were $\phi$ is the work function of the sample. Measuring $E_{K i n}$ for known $h \nu$ and $\phi$ one can obtain the binding energy of the electrons from (2.4). Since inelastic scattering reduces $E_{K i n}$ of the electrons and the mean free path of electrons in a solid is very short (10-100 $\AA$ ) XPS is a surface sensitive method and has to be done under UHV conditions. A schematic setup of a XPS spectrometer is shown in Fig. 2.3. The x-ray energy of the source used is typically $1486.6 \mathrm{eV}\left(\mathrm{Al}-\mathrm{K}_{\alpha}\right)$ or $1253.6 \mathrm{eV}\left(\mathrm{Mg}-\mathrm{K}_{\alpha}\right)$ and the binding energy accessible by XPS is therefore $E_{B}<1486 \mathrm{eV}$. The kinetic energy of the emitted electrons is measured with an electron energy analyzer. The most common type is a hemispherical analyzer. The emitted electrons passing the lens system are deflected by the negatively charged hemispheres. Depending on the potential between the two hemispheres only electrons with a certain 
energy can reach the exit slit. With hemispherical analyzers the kinetic energy of electrons can be determined with an accuracy better than $10 \mathrm{meV}$. Due to the unique energy level scheme of the elements the measured binding energy pattern can be assigned to the chemical composition of the sample. Because the binding energies of the elements can vary depending on the chemical environment one can also obtain information about the bonding and the oxidation state. In principal the intensity of the observed peaks allows to calculate the stoichiometry of the sample, but the accuracy is usually poor and only within $10-20 \%$, because the peak area is determind by many factors like subshell ionization cross section, mean free path of electrons, transmission function of analyzer and inelastic background. The absolute sensitivity of XPS is high and is in the range of $0.1-1 \%$ for most elements. The in situ XPS spectrometer used in this work provides a method to prove the purity with high sensitivity and to ensure that the samples are free of contaminations.

\subsection{Magnetometry}

The macroscopic magnetic properties of the samples were measured with a vibrating sample magnetometer (VSM) or a SQUID magnetometer. In a VSM (Fig. 2.4) the sample moves periodically between a set of detection coils and the voltage induced by the magnetic dipole field of the sample is proportional to the magnetic moment. Two subsets of detection coils are inversely arranged in order to cancel out the induction by the varying external field that is applied by a superconducting split-coil magnet. With the VSM magnetometer one can measure hysteresis curves $M(H)$ and the temperature dependence of the magnetization $M(T)$ in a field of up to $50 \mathrm{kOe}$ and in a temperature range of $5-300 \mathrm{~K}$. The the principal setup of a SQUID magnetometer is equal besides the detection of the magnetic moment. In a SQUID the magnetic flux from the sample is measured with a superconducting quantum interference device that is basically a current loop containing two Josephson contacts. The advantage of a SQUID magnetometer is its high sensitivity compared to other magnetometric methods. Magnetic moments down to $10^{-8}$ emu are detectable. 


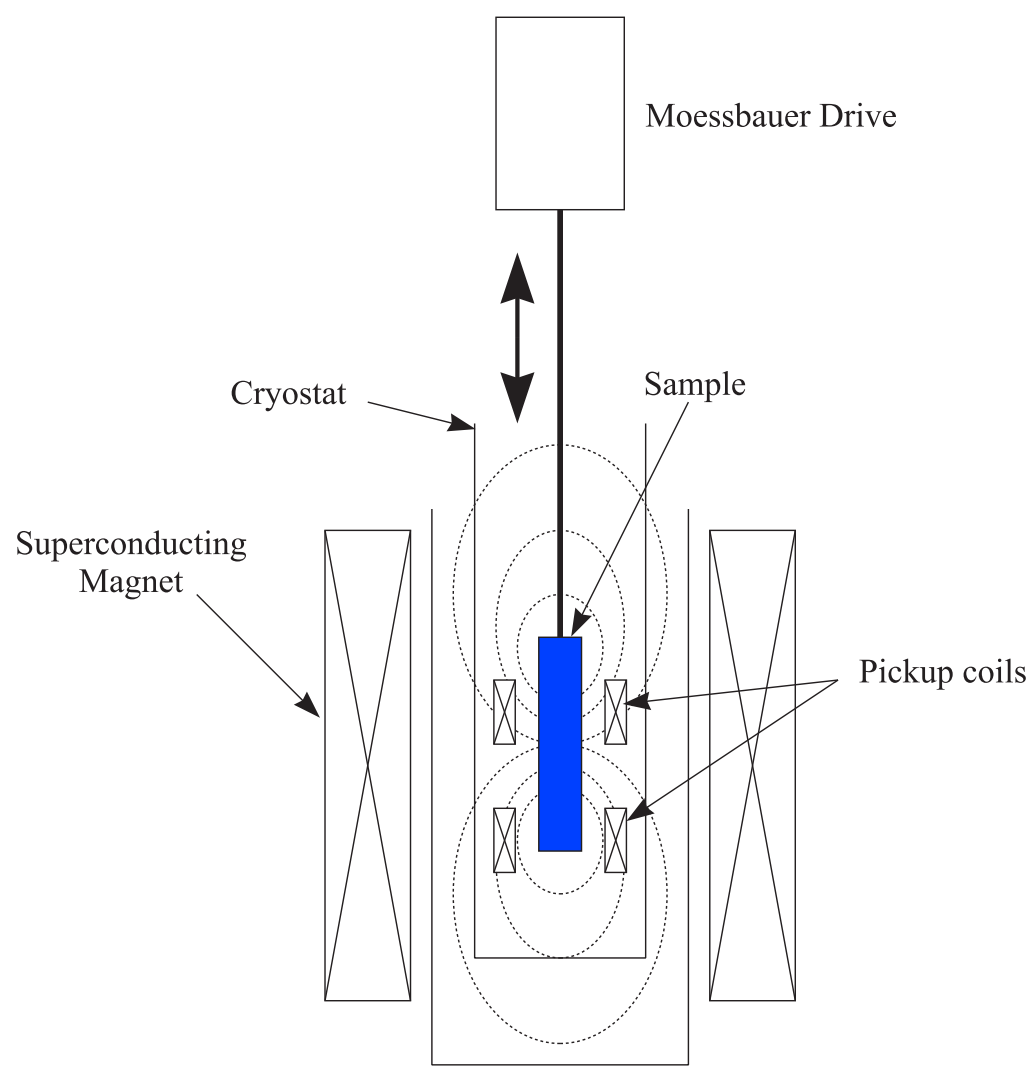

Figure 2.4: Schematic view of a vibrating sample magnetometer. 


\section{Chapter 3}

\section{X-Ray Magnetic Circular Dichroism}

\subsection{X-ray absorption}

The x-ray absorption in solids is described by the Lambert-Beer law

$$
I(E, d)=I_{0} e^{-\mu(E) d}
$$

where $I_{0}$ is the initial and $I$ the intensity after the absorption on a length $d$. The absorption coefficient $\mu$ depends on the material and the photon energy $E$. In the x-ray regime $\mu$ decreases with $E^{-7 / 2}$. When $E$ is equal to the binding energy of the electrons in an atomic shell the absorption shows a huge jump, because the photons can excite the electrons of the specific level above the Fermi level. The energy levels are element specific and are described by Moseley's law. An absorption edge is labeled by the principal quantum number of the core state $n=1,2,3 \ldots$ with the capital letters $\mathrm{K}, \mathrm{L}, \mathrm{M}, \ldots$ with an index describing the spin-orbit split of the core levels. For the $\mathrm{L}_{23}$-edge the $2 p_{1 / 2}, 2 p_{3 / 2}$ and for the $\mathrm{M}_{45}$-edge the $3 d_{1 / 2}, 3 d_{3 / 2}$ electrons are excited to the empty conduction-band states $\rho(E)$ above the Fermi energy. The absorption $\mu(E)$ is due to Fermi's golden rule

$$
\mu(E)=\omega \rho(E)
$$


and the transition probability $\omega$ depends on the overlap of the wave functions of the core level state and the valence state. The dipole selection rule requires a change in the orbital momentum $\Delta l= \pm 1$, where a change by -1 has a small probability compared to $\Delta l=+1$ [12]. Due to this fact the L-edge absorption is dominated by the $p \rightarrow d$ absorption channel and is a measure of the number of d-holes.

\subsection{XMCD Spectroscopy}

The x-ray magnetic circular spectroscopy is a x-ray absorption method that provides information about the magnetic polarization of the valence states in a solid. The magneto-optical absorption spectrum was first calculated by Erskine and Stern in 1975 [13]. More than ten years later in 1987 the first experimental observation of the XMCD was reported by Schütz et al. [14]. A brief review of the XMCD effect is given in the following.

In a ferromagnetic transition metal the magnetic properties are generated by the $3 \mathrm{~d}$ electrons in the conduction band. In the Stoner model the spinmagnetic moment is represented by the difference of the spin up and spin down electron (hole) density $m_{s} \sim \rho^{\uparrow}-\rho^{\downarrow}$. With XMCD spectroscopy one can probe $\rho^{\uparrow}$ and $\rho^{\downarrow}$ separately by x-ray absorption with circularly polarized $\mathrm{x}$-rays. For right (left) circularly polarized light, the angular momentum of the photon is parallel (antiparallel) to the x-ray propagation direction and the momentum transfered to the photoelectron is opposite for left and right circularly polarized light. If the photoelectron originates from a spin-orbit split core state (e.g. $2 p_{1 / 2}, 2 p_{3 / 2}$ ) the angular momentum of the photon can be transfered to the spin of the exited electron through the spin-orbit coupling. Assuming that the transition probabilities for spin up and spin down are equal with (3.2) the x-ray absorption for the different polarizations are

$$
\begin{aligned}
& \mu^{+}(E)=\omega \rho^{\uparrow}(E) \\
& \mu^{-}(E)=\omega \rho^{\downarrow}(E)
\end{aligned}
$$

and the difference $\mu^{+}(E)-\mu^{-}(E)$ is a measure of the magnetic polarization of the probed valence states. Depending on the absorption edge the elementally 

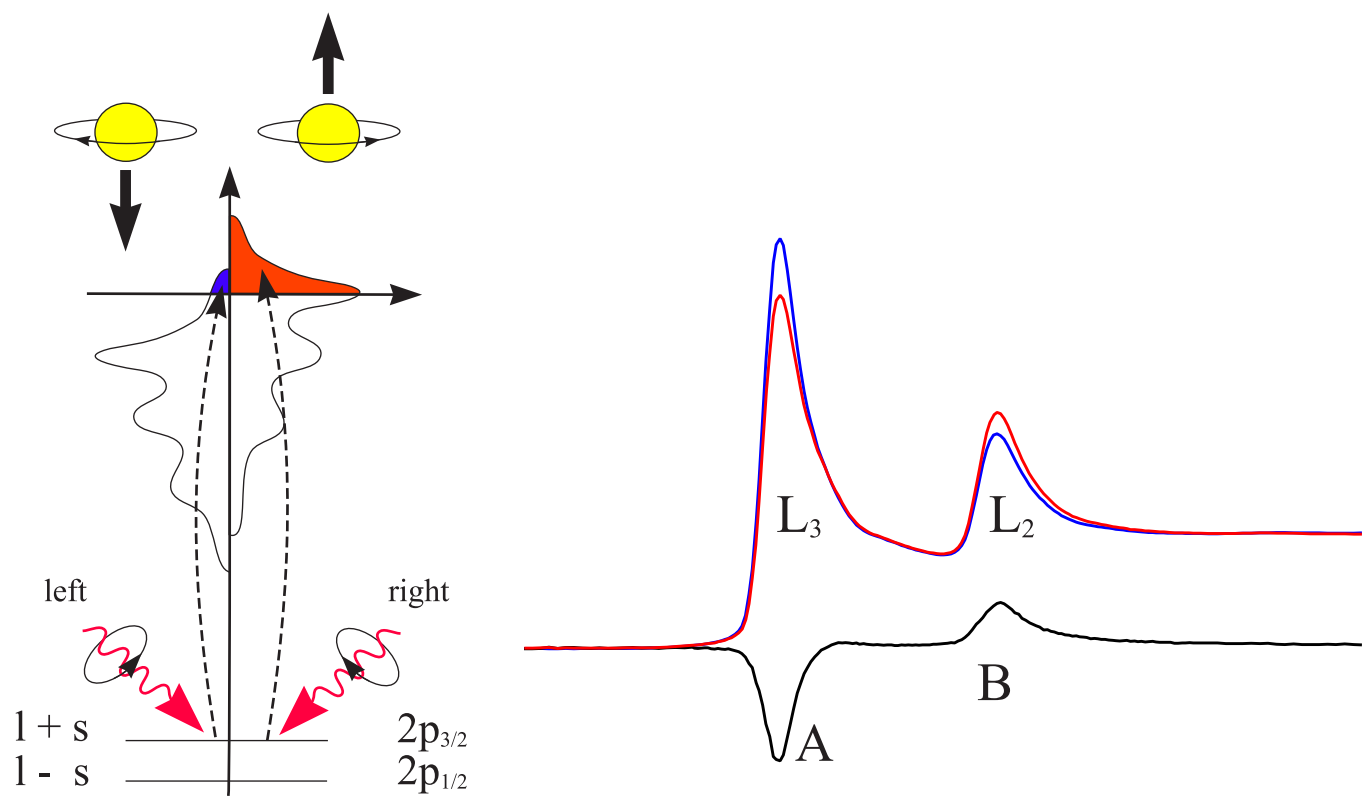

Figure 3.1: L-edge x-ray magnetic circular dichroism in the one electron picture. The transitions occur from the spin-orbit split $2 \mathrm{p}$ core shell to the empty conduction band states. The XMCD (black curve) is the difference between the absorption spectra at the $\mathrm{L}_{3}$ - and the $\mathrm{L}_{2}$-edges (red and blue curve)

resolved polarization of different valence states is observable. With the Kedge absorption $(s \rightarrow p)$ one probes the polarization of the valence p-states. The $\mathrm{L}_{23}$-edges probe the d-states and the $\mathrm{M}_{45}$-edges the f-states. Therefore the technique is not only element specific, one gains additionally information about the polarization of the different valence electron states. The difference at the absorption spectra for right (left) circular polarized light over the $\mathrm{L}_{3}$ and $\mathrm{L}_{2}$-edge are the integrated XMCD intensities $\mathrm{A}$ and $\mathrm{B}$ in Fig. 3.1. $\mathrm{A}$ and $\mathrm{B}$ have the opposite sign due to the opposite spin-orbit coupling of the $p_{3 / 2}$ and $p_{1 / 2}$ levels. Thole [15] and Carra [16] have derived sum rules to obtain the spin and orbital moment from the XMCD. A review of the XMCD effect and the sum rules can be found in [17]. The spin moment is derived from $A-2 B$ and the orbital moment from $A+B$. The sum rules are a powerful tool for magnetic investigations, but since they are derived from a one-electron picture they are not an universal tool to obtain the spin and orbital moment of a solid. A more elementary description of the sum rules was given by Altarelli [18]. An experimental confirmation about applying the sum rules 
to iron and cobalt can be found in [19].

For the magnetic transition metals and their compounds the sum rules are of good use and one can obtain elemental resolved the spin and orbital magnetic moments from XMCD experiments. But there exist limitations for the sum rules, because electron-electron interactions can alter the transition probabilities and the one electron picture does not hold anymore. For example, in the Rare Earths (RE) the 4 f-electrons have a strong Coulomb interaction with the $5 \mathrm{~d}$ electrons and the sum rules fail since the XMCD intensities do not only depend on the 5 d polarization any more. An effect called 'breathing' was first observed by Harmon and Freeman [20] for the $\mathrm{L}_{23^{-}}$ edges of Gd and is described shortly here. The probability for the $2 p \rightarrow 5 d$ electric dipole transition is proportional to the overlap of the $2 \mathrm{p}$ initial states and the $5 \mathrm{~d}$ radial part in real space. The $4 \mathrm{f}-5 \mathrm{~d}$ exchange in $\mathrm{Gd}$ causes a spin split $5 \mathrm{~d}$ band by the half filled $4 \mathrm{f}$ shell and the $5 \mathrm{~d}$ wave function with spin parallel to the $4 \mathrm{f}$ is pulled radially inwards. This leads to an enhanced transition probability for one spin channel. This would give approximately an effective polarization

$$
\begin{aligned}
p & =\frac{\omega^{\uparrow} \rho^{\uparrow}-\omega^{\downarrow} \rho^{\downarrow}}{\omega \rho} \\
& =\frac{1}{2}\left(\frac{\Delta \omega}{\omega}+\frac{\Delta \rho}{\rho}\right)
\end{aligned}
$$

A positive net $5 \mathrm{~d}$ moment implies that there are more unoccupied spin down states and $\rho^{\downarrow}>\rho^{\uparrow}$. In case of Gd the XMCD signal has actually the wrong sign, since $M^{\uparrow} \rho^{\uparrow}>M^{\downarrow} \rho^{\downarrow}$ due to the 20-30\% enhanced $M^{\uparrow}$ [21]. The magnitude of the effect scales with the $4 \mathrm{f}$ spin momentum and the d-f Coulomb interactions can vary over the RE series from Ce to Lu. Therefore one observes a multitude of different $\mathrm{L}_{2}$ - and $\mathrm{L}_{3}$-edge XMCD intensities [22] and espacially for the RE L-edges the sum rules do not give satisfactory results. 


\subsection{Beamline description}

The XMCD experiments in this work were done at several different beamlines at the storage rings of $\mathrm{LURE}^{1}, \mathrm{ESRF}^{2}$ and BESSY ${ }^{3}$. The x-ray absorption from a solid can be measured with different experimental setups. In transmission the intensity of the x-ray behind the sample is directly measured with a photodiode. Depending on the energy range of the x-rays the thickness of a sample (including substrate) is limited to $1-2 \mu m$ for $h \nu<1-2 \mathrm{keV}$ or $50-100 \mu \mathrm{m}$ for $h \nu>5 \mathrm{keV}$. The advantage of the method is the direct measurement of the absorption in a large volume. If the sample and/or substrate are to thick to be transmitted by the x-rays the total electron yield (TEY) or the fluorescence yield modes are applicable. The excited electrons produced by the absorbed photons recombine to lower energy levels and the difference in energy can emit electrons (secondary electrons, Auger electrons) or photons (fluorescence). In total electron yield the sample current caused by the emitted electrons is measured. Due to the rather easy signal detection and the possibility to use bulk samples this method is very common. But the method cannot probe a volume comparable to the transmission and can be affected by saturation effects falsifying the measured absorption. For low $\mathrm{x}$-ray energies the probing depth is only several tens of $\AA$ due to the short mean free path of the electrons in a solid. The total fluorescence yield mode has a greater probing depth than TEY but the intensity, especially for low $\mathrm{Z}$ elements, is small and the detection has smaller signal-to-noise ratio.

At a synchrotron the x-ray beam is usually generated by an insertion device like undulator or wiggler and the beam contains a spectral range $\Delta E$. The energy of the probing beam is selected by a monochromator (double crystal or grating) and the absorption $\mu(E)$ is given by

$$
\mu=\ln \left(\frac{I_{0}}{I}\right)
$$

\footnotetext{
${ }^{1}$ Laboratoire pour l'Utilisation du Rayonnement Electromagnétique, Centre Universitaire Paris-Sud, France

${ }^{2}$ The European Synchrotron Radiation Facility, Grenoble, France

${ }^{3}$ Berliner Elektronenspeicherring-Gesellschaft für Synchrotronstrahlung, Berlin, Germany
} 


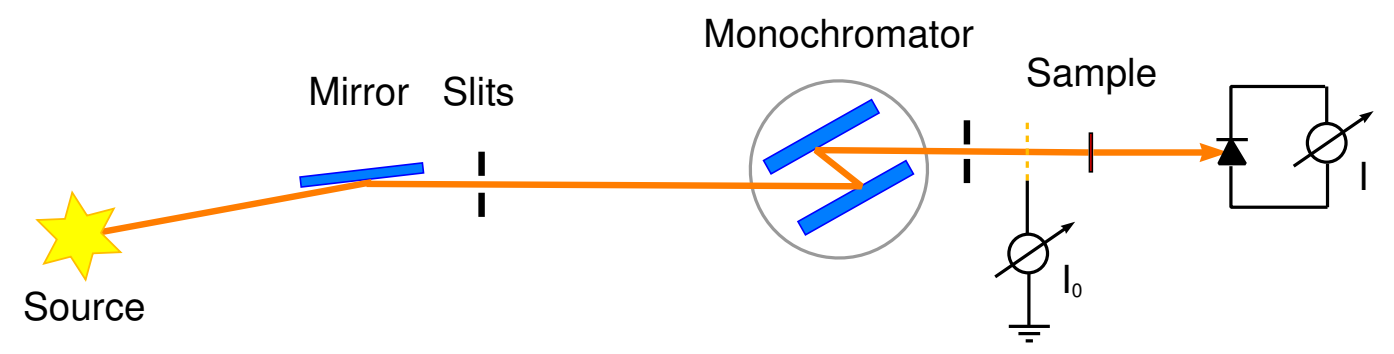

Figure 3.2: Beamline with a double crystal monochromator. The absorption is measured with a photodiode

where $I_{0}$ is proportional to the intensity of the x-ray beam in front of the sample. An absorption spectrum is recorded by scanning the energy with the monochromator over the desired range and measuring the absorption at each energy step. The scanning monochromator technique was used at the beamlines SU23 (LURE) for the Fe L-edges, SU22 (LURE) for the Gd Medges and at the PM3 (BESSY) for the N K-edge. The energy resolution for those beamlines is typically in the range of $0.1 \mathrm{eV}$. At the beamlines SU22 and SU23 the absorption was measured in transmission with samples on mylar substrates. At the PM3 the N K-edge absorption was measured in the total electron yield mode. The XMCD can determined by the difference of the absorption spectra $\mu^{+}$and $\mu^{-}$. In chapter 3 the absorption spectra $\mu^{+}$ and $\mu^{-}$were defined as the absorption by circular polarized light of opposite helicities, but it is can be shown that the reversal of magnetization of the sample with an external magnetic field at a constant helicity has the same effect [23]. For practical reasons in the majority of cases the magnetic field is flipped instead of the helicity of the x-rays.

At a dispersive beamline the x-ray beam is reflected by a bent Si crystal instead of using a monochromator (Fig. 3.3). Depending on the reflection angle and the amount of bending an energy range $\Delta E$ is focused on the sample and the transmitted XAS spectrum is measured by a CCD sensor array simultaneously. The spatial resolution of the detector (and its distance from the focal point) determines the energy resolution of the recorded spectrum. This method is obviously only available in transmission. The simultaneous measurement of the whole XAS spectrum is an advantage for time resolved experiments. At the dispersive beamlines D11 (LURE) and ID24 (ESRF) 


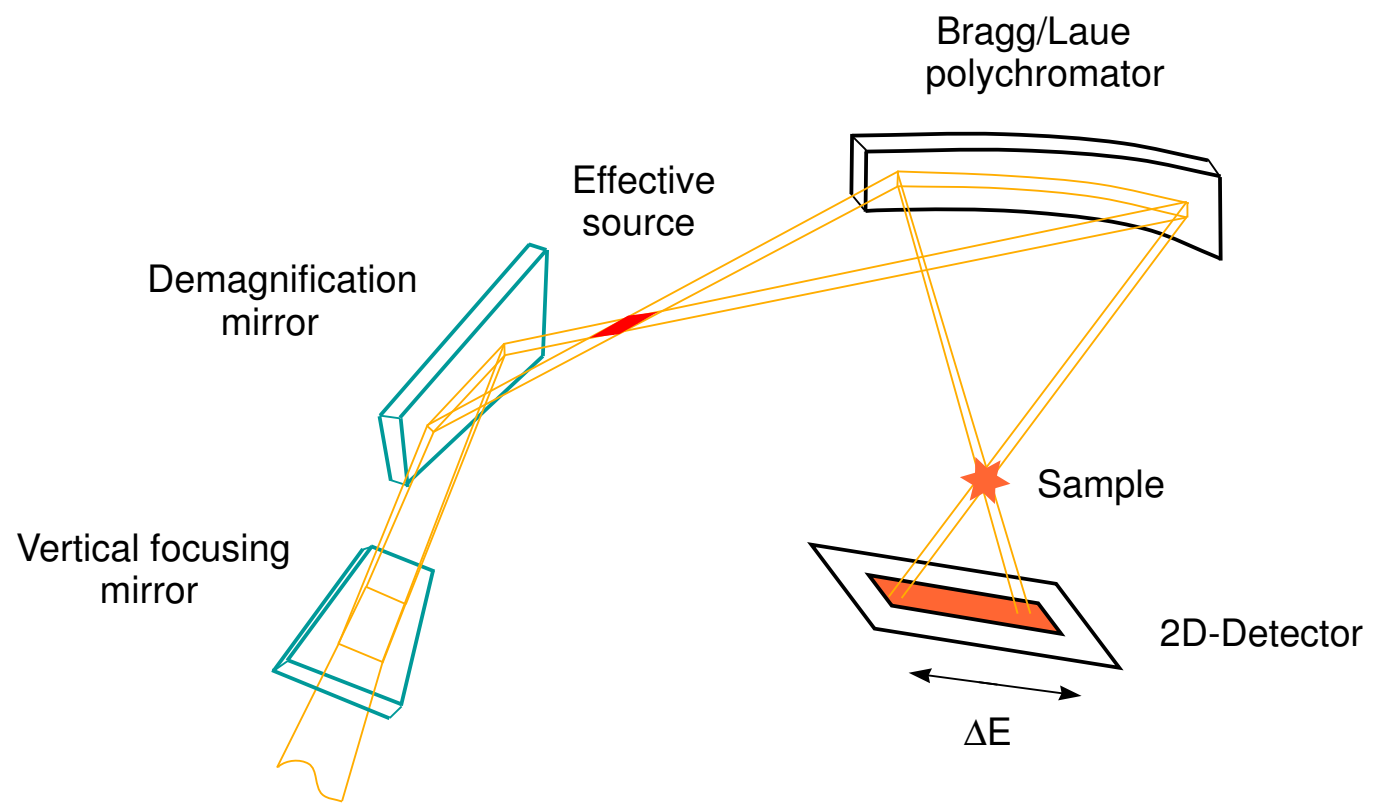

Figure 3.3: Dispersive beamline optic. The energy range $\Delta E$ is measured immediately. All angles are extreme overdone for clarification

the Gd L-edges were measured with samples on Kapton foils. The energy resolution at the Gd L-edges is typically $0.2-0.3 \mathrm{eV}$. Both beamlines have in principal the same setup. The D11 uses the natural polarization of the x-rays above the electron orbit of the storage ring, whereas the ID24 uses linearly polarized light and a diamond quarter wave plate (QWP) to achieve either right or left circularly polarized light. The quarter wave plate is a $700 \mu \mathrm{m}$ thick diamond plate that is inserted into the linearly polarized beam in Laue geometry close to the (111) reflection. Due to the birefringent properties of the diamond plate in this special geometry the polarization of the beam is circular after passing the QWP. A detailed description of the beamline is found in [24]. 


\section{Chapter 4}

\section{Rare Earth Pnictides}

\subsection{Rare Earth Magnetic Semiconductors}

Pnictogenes is a uncommon description for the group-V elements (N,P ...) and the (binary) compounds based on pnictogenes are called pnictides. The notion 'pnictides' was commonly used for the binary alloys between the RE (or Actinides) and the group-V elements. Another prominent class of binary alloys with the RE and group-VI elements (O,S...) are called the 'chalcogenides' and were also subject of rich investigations around the 60's and 70's. Great attention during this time was payed to $\mathrm{EuO}$ as a ferromagnetic semiconductor, which can be seen as a prototype of these compounds. Since the chemical properties of the Rare Earths are similar the chalcogenides and the pnictides have many common properties. For example, the chalcogenides $\mathrm{EuO}, \mathrm{EuS}, \mathrm{EuSe}$ and EuTe have the same lattice structure as the pnictides GdN, GdP, GdAs and GdSb. All of them crystallize in a $\mathrm{NaCl}$ (rocksalt) structure with the symmetry group Fm3m. Due to the increasing ionic radius in the series O...Te and N...Sb the lattice parameter of the chalcogenides and the pnictides grows accordingly. This is often accounted for the fact that the magnetic ordering changes from ferromagnetic $(\mathrm{EuO})$ to antiferromagnetic (EuSe) since they are supposed to be model systems for Heisenberg magnets [25]. The interest in the chalcogenides was founded on $\mathrm{EuO}$ that was reported to be a ferromagnetic semiconductor with a transition to a metal at $T_{C}$, reflected in a drop in the resistance over several orders of magnitude [26]. 
Significant research was done on the chalcogenides and also the pnictides, especially $\operatorname{GdX}(\mathrm{X}=\mathrm{N}, \mathrm{S}, \ldots)$, were in the scope of research since they are comparable to the chalcogenides with similar properties. Since about 1970 the magnetic and electronic properties of GdN were discussed controversially. The material was reported to be a ferromagnet as well as an antiferromagnet. Later it was found that GdN is ferromagnetic [27,28,29]. Theoretical models about how the magnetic ordering is connected to the electron structure in the pnictides were proposed by Kasuya $[30,31]$ and Hasegawa [32]. Nowadays the field of magnetoelectronics (spintronics) restarted the activity on the chalcogenides and pnictides, since they may possibly be used as spinfilter devices with a spin polarization of $100 \%$. A sucsessful implemetation of the chalcogenides or pnictides as a spinfilter could be an intersting step in the field of spintronics since they are semiconductors. Recent investigations showed that the spin injection from a metallic ferromagnet into a semiconductor always suffers from the resistance missmatch between the materials [2,1]. Therefore a semiconducting ferromagnet is possibly a good candidate to enhance the effective spin injection into a semiconductor. From a technolocical point of view the low $T_{C}$ of the materials is a disadvantage because the ordering temperature of all chalcogenides and pnictides are below $77 \mathrm{~K}$ (liquid nitrogen) but doping (e.g. EuO:Gd) can raise $T_{C}$ effectivley [25]. With the increasing importance of microelectronics the semicondcutor GaN has also attracted a lot of interest in order to combine spintronics with microelectronic techniques. An attempt to add ferromagnetism to a semiconductor are the so called diluted magnetic semiconductors (DMS) like $\mathrm{Ga}_{1-x} \mathrm{Mn}_{x} \mathrm{~N}$, where the Mn content is about $3-12 \%$. A recently published work reported a $T_{C}$ of $400 \mathrm{~K}$ for Gd doped $\mathrm{Ga}_{1-x} \mathrm{Gd}_{x} \mathrm{~N}$ [5]. Althoug the Gd concentration in $\mathrm{Ga}_{1-x} \mathrm{Gd}_{x} \mathrm{~N}$ is only in the order of $1 \%$ the clarification of the magnetic exchange in GdN may give useful hints for the exchage coupling in semicondcuting alloys like $\mathrm{Ga}_{1-x} \mathrm{Gd}_{x} \mathrm{~N}$. 


\subsection{Electronic structure of the Rare Earth Pnictides}

The magnetic coupling in Rare Earth (RE) metals is commonly understood by a RKKY exchange via the conduction electrons in the RE s,p,d bands. A strong intra-atomic $5 \mathrm{~d}-4 \mathrm{f}$ spin coupling connects the RKKY exchange to the localized RE 4 f electrons that do not have a direct overlap between the RE sites. In ferromagnetic RE semiconductors like $\mathrm{EuO}$ or $\mathrm{GdN}$ the conduction band is nearly empty. A suggestion about the mechanism of magnetic exchange in the RE semiconductors was given by Methfessel [33]. In this model (Fig. 4.1) a direct ferromagnetic exchange $J_{1}$ between the $5 \mathrm{~d}-\mathrm{t}_{2 g}$ orbitals and a indirect antiferromagnetic exchange $J_{2}$ between the $\mathrm{Gd} 5 \mathrm{~d}-\mathrm{e}_{g}$ and the $\mathrm{N}$ 2 p orbitals is responsible for the magnetic properties. Both $J_{1}$ and $J_{2}$ vary with the interatomic distance, $J_{1}$ more sensitively than $J_{2}$. Since $J_{1}$ drops much faster with increasing distance the magnetic coupling in the series $\mathrm{EuO}$, EuS, EuSe ... changes from ferromagnetic to antiferromagnetic due to the increasing unit cell [34]. This is a very localized picture that belongs more to an insulator and the true electronic state depends on the amount of overlap between the orbitals and their amount of localization.

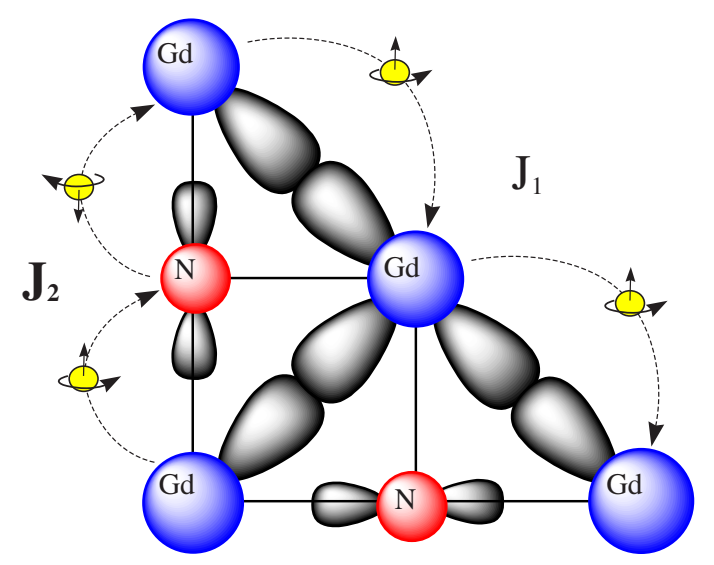

Figure 4.1: Magnetic exchange coupling $J_{1}$ and $J_{2}$ in a localized Heisenberg picture. The ferromagnetic coupling $J_{1}$ between the nearest-neighbor Gd ions is mediated by the direct overlap of the $5 \mathrm{~d}^{-} \mathrm{t}_{2 g}$ orbitals. The antiferromagnetic exchange between the next-nearest neighbor Gd ions is a superexchange involving the $5 \mathrm{~d}-e_{g}$ and the N 2p orbitals. (after [33]) 


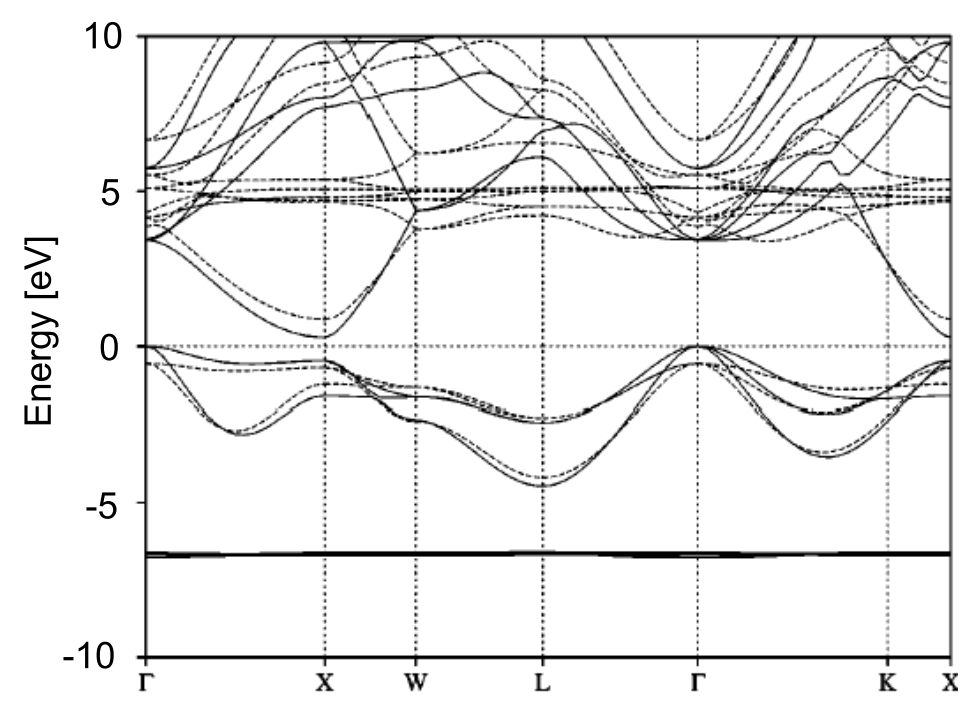

Figure 4.2: Spin polarized band structure of GdN from [35]. Within this calculation $\mathrm{GdN}$ is a semiconductor in the ground state with a indirect gap of $0.8 \mathrm{eV}$, but it is possible that the gap is smaller and closes at the $\mathrm{X}$ point when the GdN becomes ferromagnetic. Solid lines represent majority spinsbands, dotted lines minority spin-bands.

The electronic structure of the RE chalcogenides and pnictides is a challenge for theory up to now. The standard method of determining the electronic properties of magnetic materials is the LSDA (Local Spin Density Approximation). In a RE ferromagnetic semiconductor the LSDA is of limited success due the strong correlations of the $4 \mathrm{f}$ electrons. Lambrecht calculated a spin polarized band structure of GdN with a LSDA+U approach, but he has to introduce a gap correction to obtain a band gap and neglected the $4 \mathrm{f}$ correlations [35]. The result is shown in Fig. 4.2. According to this calculation GdN would be a semiconductor even below $T_{C}$ with a indirect band gap of about $0.8 \mathrm{~V}$ and a direct gap of $1.1 \mathrm{eV}$. The top of the valence band at the $\Gamma$ point is formed mainly by the $\mathrm{N} 2 \mathrm{p}$ states and the bottom of the conduction band at the $X$ point by the Gd 5 d states. Above $T_{C}$ the bands are represented by the average of the majority and minority bands and the band splitting that occurs below $T_{C}$ lowers the gap. This can be seen in optical absorption spectroscopy by a shift of absorption onset to lower energy and is called redshift. The calculated redshift is about $0.3 \mathrm{eV}$ and comparable 
to the redshift reported by Wachter for $\mathrm{EuO}$ [25]. Lambrecht stated that it would be possible that the gap closes for one spin channel only when the GdN becomes ferromagnetic, since the gap may be smaller than calculated. Therefore the groundstate of GdN might be a half-metal or a metal. Another approach was recently made by Aerts et al. by including the $4 \mathrm{f}$ correlations with an $a b$ initio self-interaction corrected LSDA [36]. They find a halfmetallic groundstate for GdN. In this case GdN would be a very interesting material due to the $100 \%$ spin polarization and might be a suitable system to study the spin injection into semiconductors.

\subsection{Preparation and structure of GdN}

The preparation of all GdN thin films was carried out in the UHV-chamber described in chapter 2 using various substrates like Si, Sapphire, Mylar ${ }^{\top M}$ and Kapton ${ }^{\top \mathrm{N}}$. The samples were grown by reactive sputtering at a nitrogen pressure of about $2 \cdot 10^{-5}$ torr on $\mathrm{Si}(100)$ and $\mathrm{Al}_{2} \mathrm{O}_{3}$ (1120) substrates with different substrate temperatures between room temperature and $700^{\circ} \mathrm{C}$. Because $\mathrm{N}_{2}$ is an inert gas a ECR plasma source facing the sample was used to dissociate the nitrogen to improve the reaction with the growing film (see chapter 2). To avoid alloying with the $\mathrm{Si}$ a $\mathrm{Cr}$ or $\mathrm{W}$ buffer was sputtered onto the Si substrates first. It turned out that the samples always grow polycrystalline and the buffer does not have a significant effect on the crystal structure within this temperature range. The substrate temperature itself has a significant influence on the growth of the GdN films. With higher substrate temperatures the crystallinity of the films is enhanced but remains polycrystalline. In Fig. 4.3 the Bragg-Bretano x-ray diffraction patterns for various substrate temperatures of $1000 \AA$ GdN thin films on Si (100) with a $50 \AA \mathrm{W}$ buffer are shown. The first obvious difference is the increasing intensity and the enhanced sharpness of the peaks at higher substrate temperatures, indicating an increasing grain size. Using Scherrer's formula to determine the grain size from the peaks FWHM one finds a maximum average size of $100 \AA$ for a sample grown at $450^{\circ} \mathrm{C}$. At temperatures higher than $500^{\circ} \mathrm{C}$ the $\mathrm{GdN}$ starts to peel off from the $\mathrm{Si}$ substrates and becomes a tarnish surface. With sapphire substrates the deposition temperature could be 


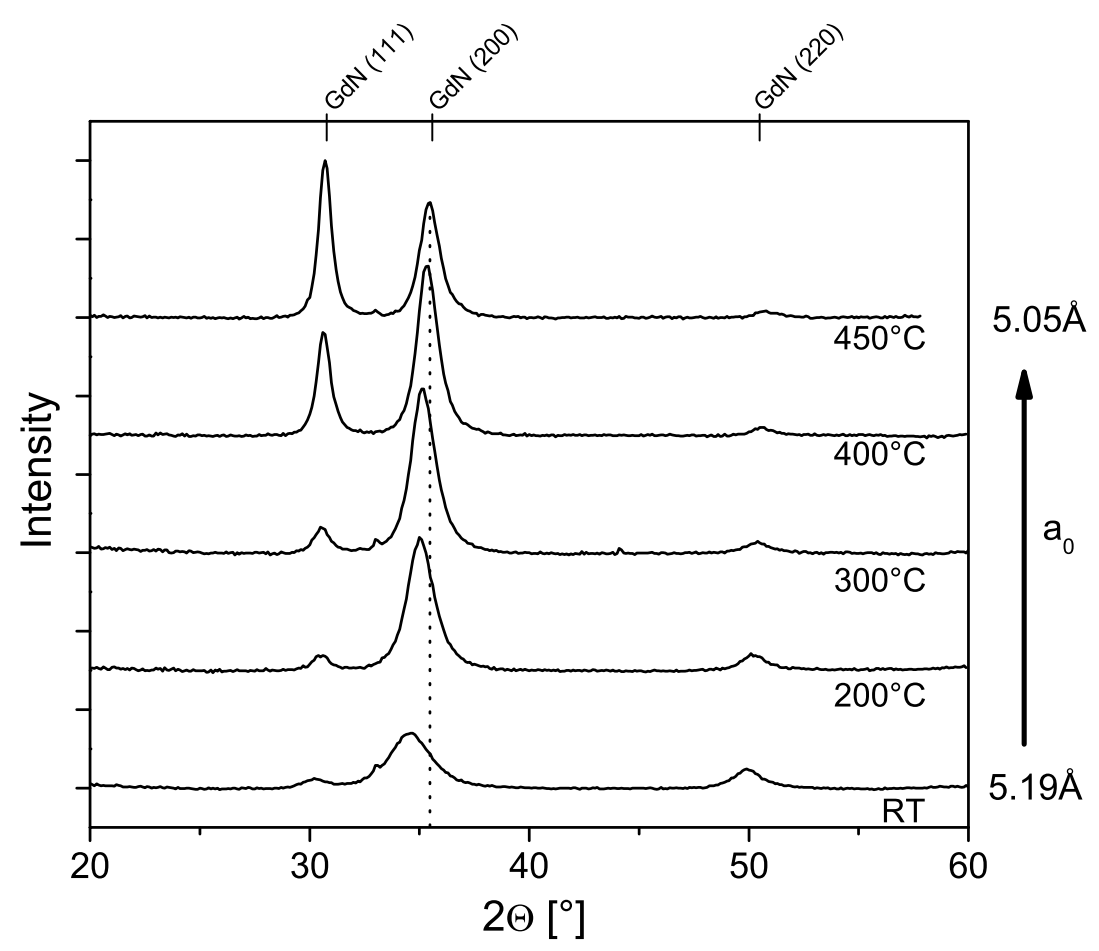

Figure 4.3: X-ray diffraction pattern of $1000 \AA$ thick GdN films grown on Si (100) at various substrate temperatures. The lattice constant $a_{0}$ decreases with higher temperatures.

raised to $700^{\circ} \mathrm{C}$ without any decomposition but the crystal structure is not improved significantly. Due to the easier handling most of the GdN thin films were grown on $\mathrm{Si}$ (100). Another issue that can be seen in Fig. 4.3 is that for substrate temperatures $T_{S}<400^{\circ} \mathrm{C}$ the average lattice constant is larger than for bulk GdN. Holleck et al. reported an increasing lattice constant in pnictides due to a nitrogen deficiency or impurities with oxygen [37]. This is because the trivalent ionic $\mathrm{Gd}^{3+}$ in $\mathrm{GdN}$ has a smaller sphere radius than the metallic bound Gd. Despite the different growth conditions one never observes any other peaks than those belonging to the $\mathrm{NaCl}$ structure of $\mathrm{GdN}$, but the intensity and the FWHM can vary depending on substrate and temperatures. Especially no peaks of hcp Gd were found. It turned out that the reactive sputter process always leads to $\mathrm{Gd}_{1-x} \mathrm{~N}_{x}$ without phases of metallic Gd. 


\subsubsection{Chemical Analysis}

Since the stoichiometry of GdN can vary in a certain limit without changing the crystal structure the x-ray diffraction patterns are not a sufficient proof for the quality of the GdN films and a chemical analysis is also necessary. Unfortunately most of the methods that are able to determine the chemical composition of thin films are not very accurate. An additional problem is the degeneration of the GdN films at ambient air making a cap layer necessary unless the analysis can be done in situ. Practicable methods that were accessible within this work are Rutherford Backscattering (RBS), X-ray Photoelectron Spectroscopy (XPS), Energy Dispersive X-ray analysis(EDX) and Resonant Nuclear Reaction Analysis (RNRA), but not all of them are a good choice for GdN thin films. RBS suffers from the fact that the ratio of the backscattered signal between Gd and $\mathrm{N}$ is very unfavorable due to the large atomic mass of the Gd compared to the nitrogen. RBS spectra would be dominated by the Gd with only a very small contribution of the nitrogen and therefore the error would be large. Due to the necessary cap layer all methods that rely on accelerated electrons (either as source or detection like EDX, XPS and Auger) are of limited use due to the comparable small mean free path of electrons in solids and the fact that a high energy electron beam can also degenerate the sample. Therefore the nitrogen concentration was determined by RNRA (see section 2.3.1). With RNRA it is possible to measure a depth profile of the nitrogen concentration in a GdN film within a length scale of several hundreds of $\mathrm{nm}$ and a cap layer is not a serious disadvantage. In Fig. 4.4 the RNRA profile of a $1000 \AA$ GdN film on Si with a $200 \AA$ Al cap layer is shown. The sample layout is shown at the top of the graph. The sharpness of the profile is noticeably broadened at the top and at the bottom of the film. This broadening does not indicate a variation of the nitrogen concentration but is caused by the energy broadening of the proton beam when traveling through the solid. The measured profile is a convolution of the real nitrogen concentration profile and the spatial energy broadening of the beam. In the film center the measured concentration is constant within the resolution of the measurement and reflects a nitrogen concentration of $50 \%$, whereby the accuracy of the method is about $5 \%$. Since the NRNA 


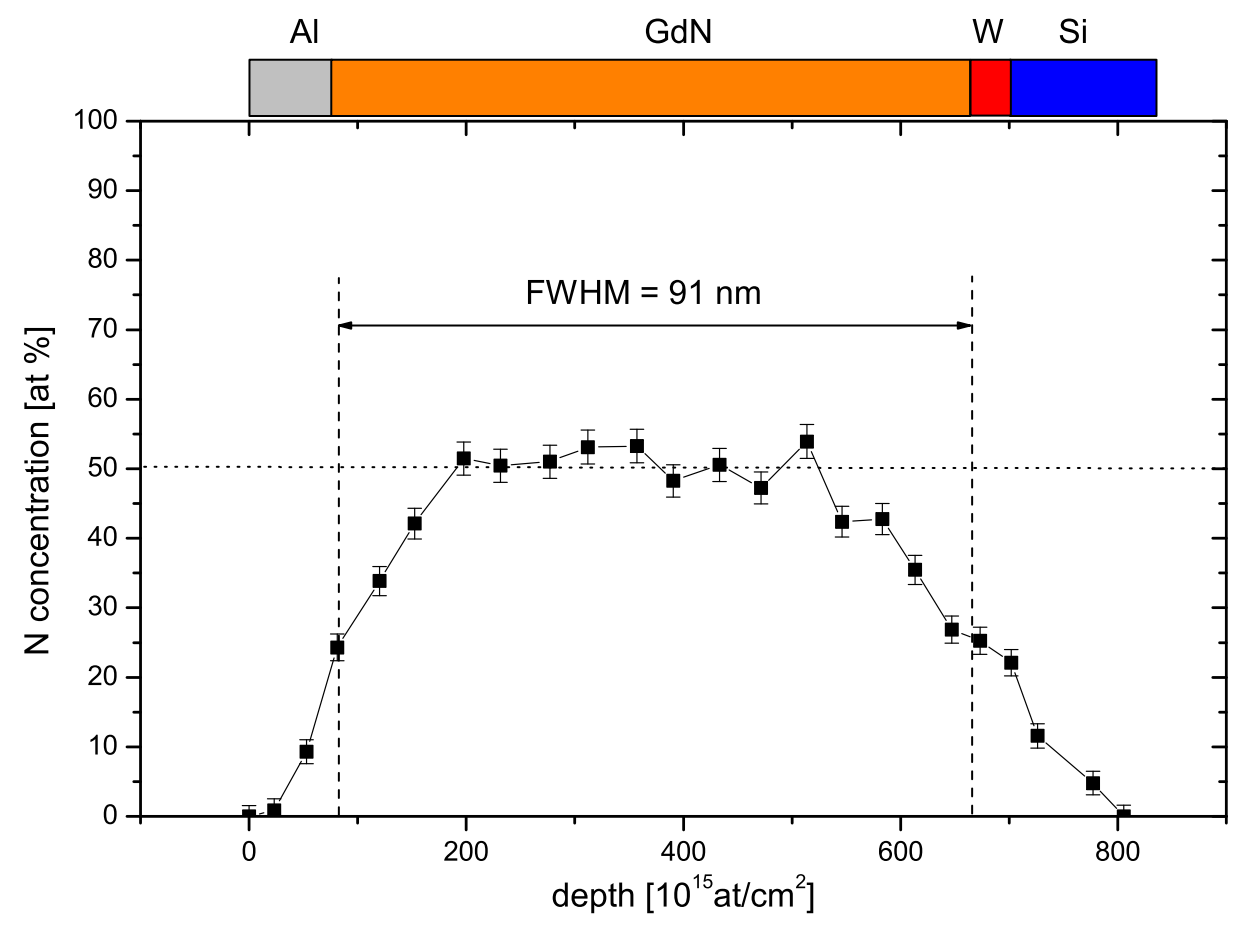

Figure 4.4: RNRA depth profile of the nitrogen concentration of a $1000 \AA$ thick GdN film with a $200 \AA \mathrm{Al}$ cap layer.

made here is only sensitive to nitrogen the profile does not contain any information about impurities like oxygen. To achieve additional information about the chemical composition of the samples in situ XPS spectra of the thin films were taken before the cap layer was added. Due to the high sensivity of XPS impurity concentrations of less than $1 \%$ would be visible in the spectra, whereas the absolute accuracy determinig the concentration is usualy poor and is in the range of 10-20\%. However, the easy oxidation of the RE makes it necessary to analyze the samples with regard to possible contamination with oxygen.

In Fig. 4.5 a survey scan (unmonochromatized $\mathrm{Al} \mathrm{K}_{\alpha}$ ) of a $250 \AA \mathrm{GdN}$ film on $\mathrm{Si}$ (100) is shown. All peaks are identified to belong either to Gd or N, besides a small peak at the O1s position $(530 \mathrm{eV})$. The O1s peak was found to increase in intensity during the measurement, indicating than the free surface of the GdN oxidizes very easily. Since the partial pressure of oxygen in the XPS chamber was always below $5 \cdot 10^{-11}$ mbar an oxidation due to the 


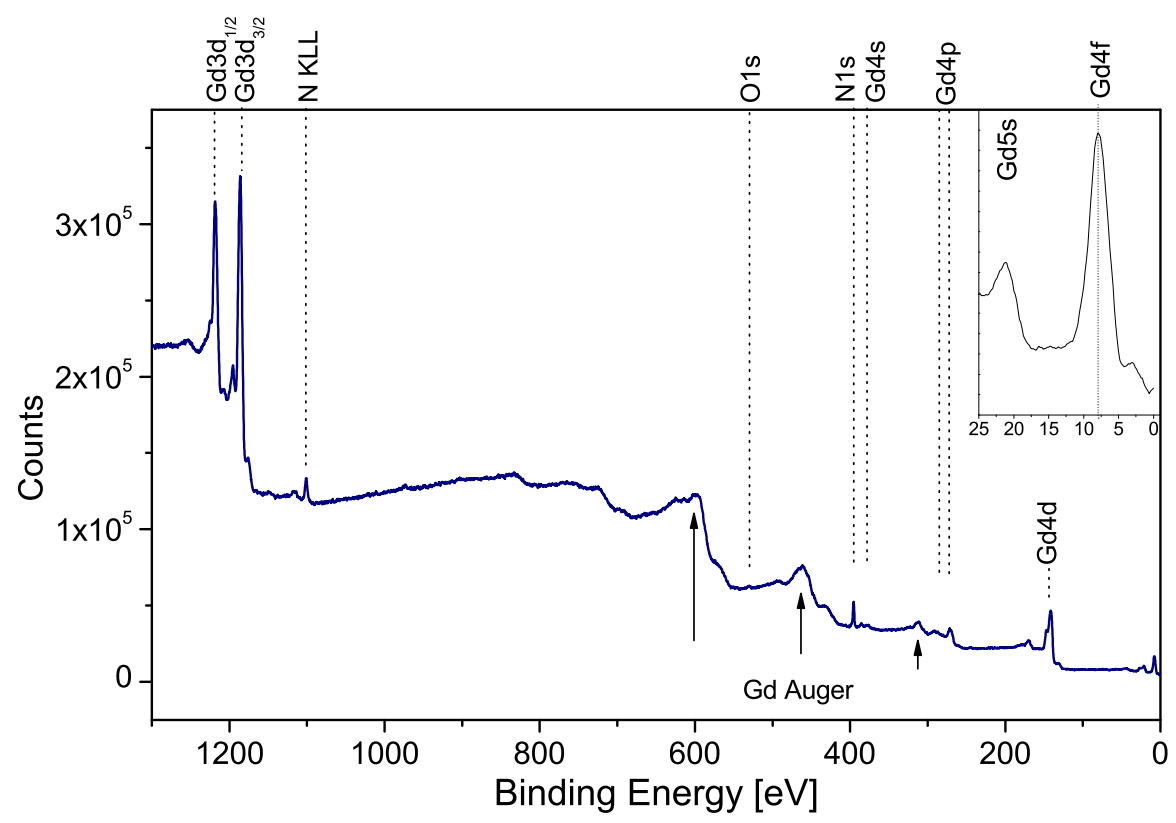

Figure 4.5: In situ XPS spectra of GdN film on Si with unmonochromatized Al$\mathrm{K}_{\alpha}$ radiation. All indentified peaks are labeled on the top. The Gd $5 \mathrm{~s}$ and $4 \mathrm{f}$ peaks are shown in the inset.

residual oxygen is unlikely. A detailed residual gas analysis showed that the operation of the x-ray source of the XPS spectrometer lead to an increase of the partial pressure of $\mathrm{CO}$ to about $1 \cdot 10^{-9}$ mbar when the emission current is switched on. At the same time the O1s peak starts to increase and it turned out that the oxidation of the GdN occurs during the XPS measurement. To estimate the concentration before the measurement a sequence in time of the evolution of the O1s and N1s peak area was done and fitted with an exponential growth. The result can be seen in Fig. 4.6. On the left side the O1s peak area is plotted against time (dots), the N1s area is shown on the right side. During the complete sequence the N1s peak barely decreases stating that this is a surface effect. The occupation of a surface should then follow a exponential growth (red line) and the extrapolation to $t=0$ is identical to the initially concentration of oxygen at the surface. As one clearly sees the initially concentration from the fit is zero and one can conclude that the oxygen impurity content (if any) is below the sensitivity of the XPS spectrometer. Although the concentration determined by XPS is usually 


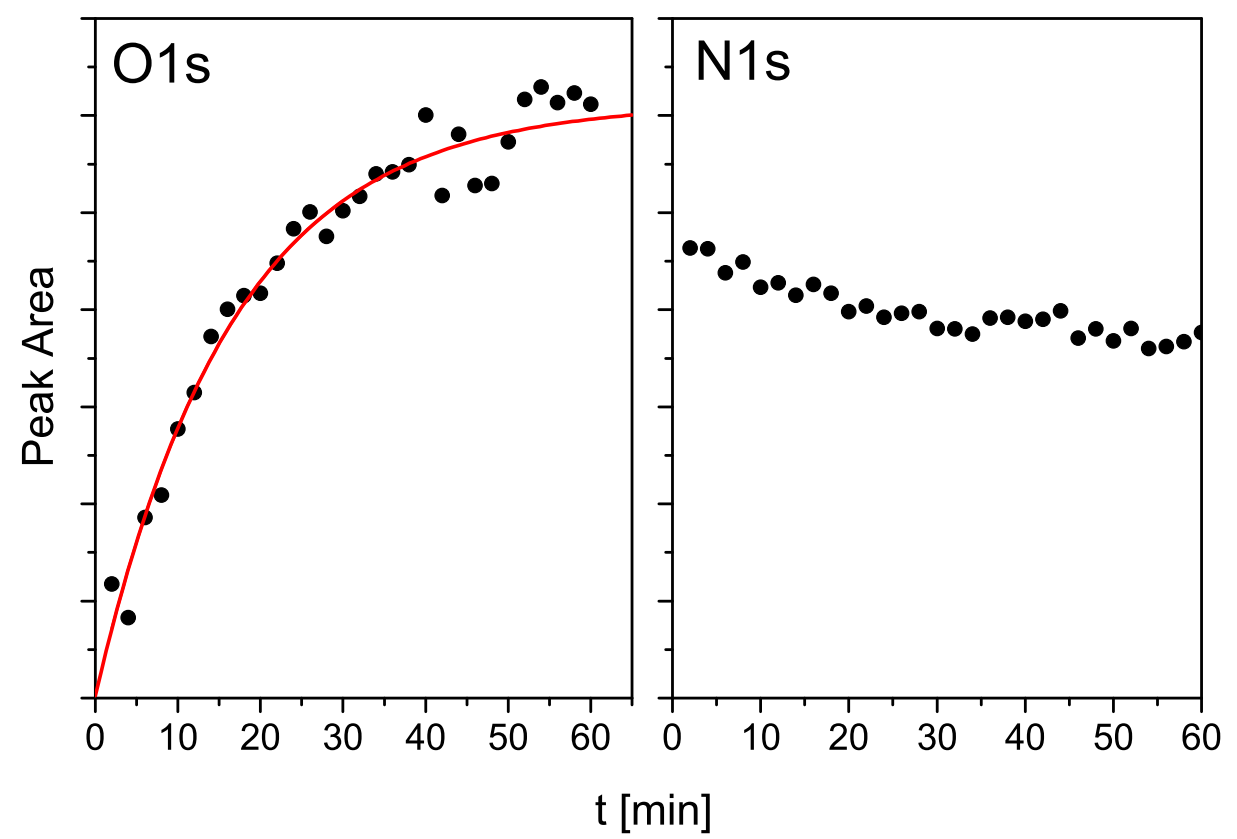

Figure 4.6: Evolution of the O1s peak area with time from a $250 \AA$ GdN film measured with XPS. On the left side the O1s peak area is plotted against time. The red curve is a fitted exponential growth. On the right side the evolution of the N1s peak area is shown.

not very accurate an analysis of the N1s and Gd4d peak gives a nominal composition of $\mathrm{Gd}_{50} \mathrm{~N}_{50}$.

For all different growth conditions (temperature, $\mathrm{N}_{2}$ pressure, sputter rate) and substrates and the resulting differences in the crystal structure (see Fig. 4.3) one never observes a significant deviation from the nominal concentration $\mathrm{Gd}_{50} \mathrm{~N}_{50}$ with the methods used in this work. Therefore a variation of the nitrogen content must be in the order a few percent or even less.

\subsection{Magnetic and electronic properties of GdN}

In the previous section the structural properties of the GdN thin films were discussed and it was found that it is possible to grow polycrystalline films with a good stoichiometry. The magnetic and electronic properties are a more important measure for the quality of the films, since deviations from the 
optimal stoichiometry or the amount of impurities that are below the detection limit of the here applied methods may affect the magnetic or electronic properties. The examination of the films with conventional magnetometry is therefore a essential proof of the key parameters of a ferromagnet, like the Curie temperature and the saturation magnetization. As seen in section 4.3

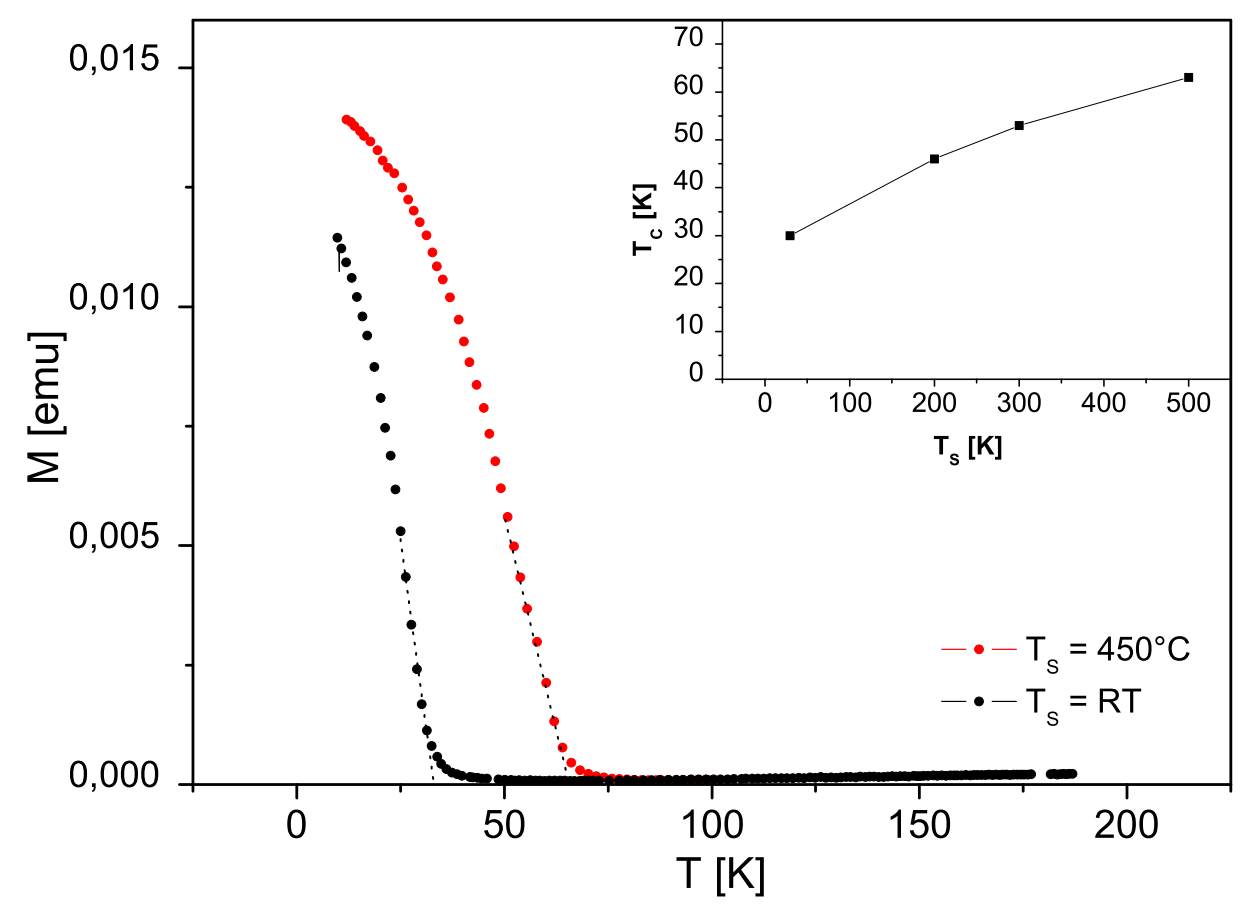

Figure 4.7: Comparison of the magnetizations in an external field of 2000e of $1000 \AA$ thick GdN samples grown a room temperature and at $450^{\circ} \mathrm{C} . T_{C}$, determined by a linear extrapolation of the magnetization, vs. substrate temperature is shown in the inset

the substrate temperature has a distinct effect on the crystal structure of the films. In Fig. 4.7 one can see a clear difference in the spontaneous magnetization of a sample grown at room temperature (black curve) and one grown at $450^{\circ} \mathrm{C}$. An estimation of the $T_{C}$ of the samples can be made by determining the intersection of a linear fit (dotted line) in the region around $T_{C}$ and the $\mathrm{x}$-axis. It yields $65 \mathrm{~K}$ for the sample grown at $450^{\circ} \mathrm{C}$ and $30 \mathrm{~K}$ for the sample grown at room temperature. As mentioned above, a significant difference in stoichiometry was never observed within the accuracy of the here applied methods. We attribute the low $T_{C}$ of the samples grown at room temperature 


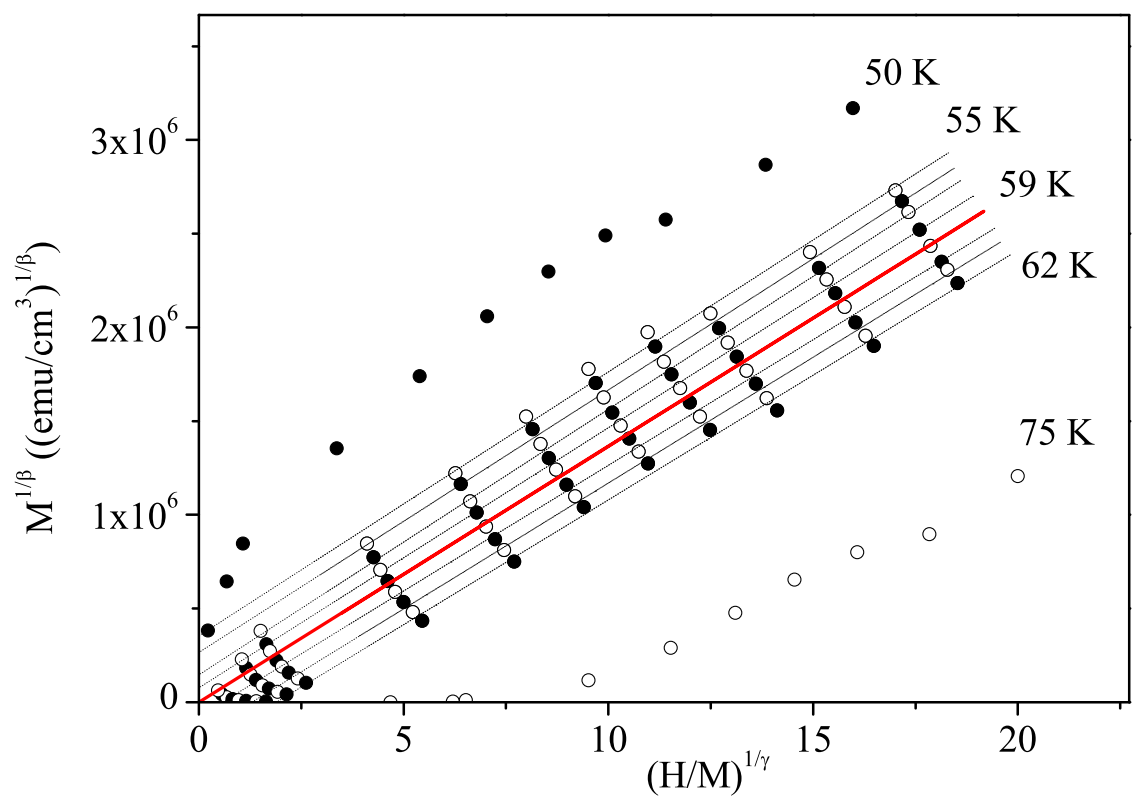

Figure 4.8: Determination of $T_{C}$ with a Arrot-Plot of a $500 \AA$ GdN film. The $T_{C}$ of $59 \mathrm{~K}$ is in very good agreement with the bulk value. (after [38])

to the increased lattice constant since a greater next-neighbor distance in the picture of Methfessel causes a strong decrease of the ferromagnetic exchange constant $J_{1}$, but only a small reduction of the antiferromagnetic exchange constant $J_{2}$.

A $T_{C}$ of $65 \mathrm{~K}$ might be to high compared to the $58 \mathrm{~K}$ reported in literature [29]. However, the determination of $T_{C}$ by a linear extrapolation of the magnetization in Fig. 4.7 is not very reliable. In order to determine the $T_{C}$ more precisely a series of $\mathrm{M}(\mathrm{H})$ curve were measured at various temperatures and plotted in an Arrott-Plot. [38]. With the procedure proposed by Arrott the critical exponents $\beta$ and $\gamma$ near the critical point $T_{C}$ of a ferromagnet can be determined from magnetization vs. external field $(M(H))$ measurements at various temperatures. In a plot $(M)^{1 / \beta}$ vs. $(H / M)^{1 / \gamma}$ the $M(H)$ curves are straight lines for temperatures near $T_{C}$, and $T_{C}$ is marked by the line crossing the origin of the plot $[39,40]$. In Fig. 4.8 an Arrott-Plot of a $500 \AA$ thick GdN film grown at $450^{\circ} \mathrm{C}$ on a $\mathrm{Si}(100)$ substrate is shown (from [38]). The red line through the origin marks a $T_{C}$ of $59 \mathrm{~K}$, which is in very good 

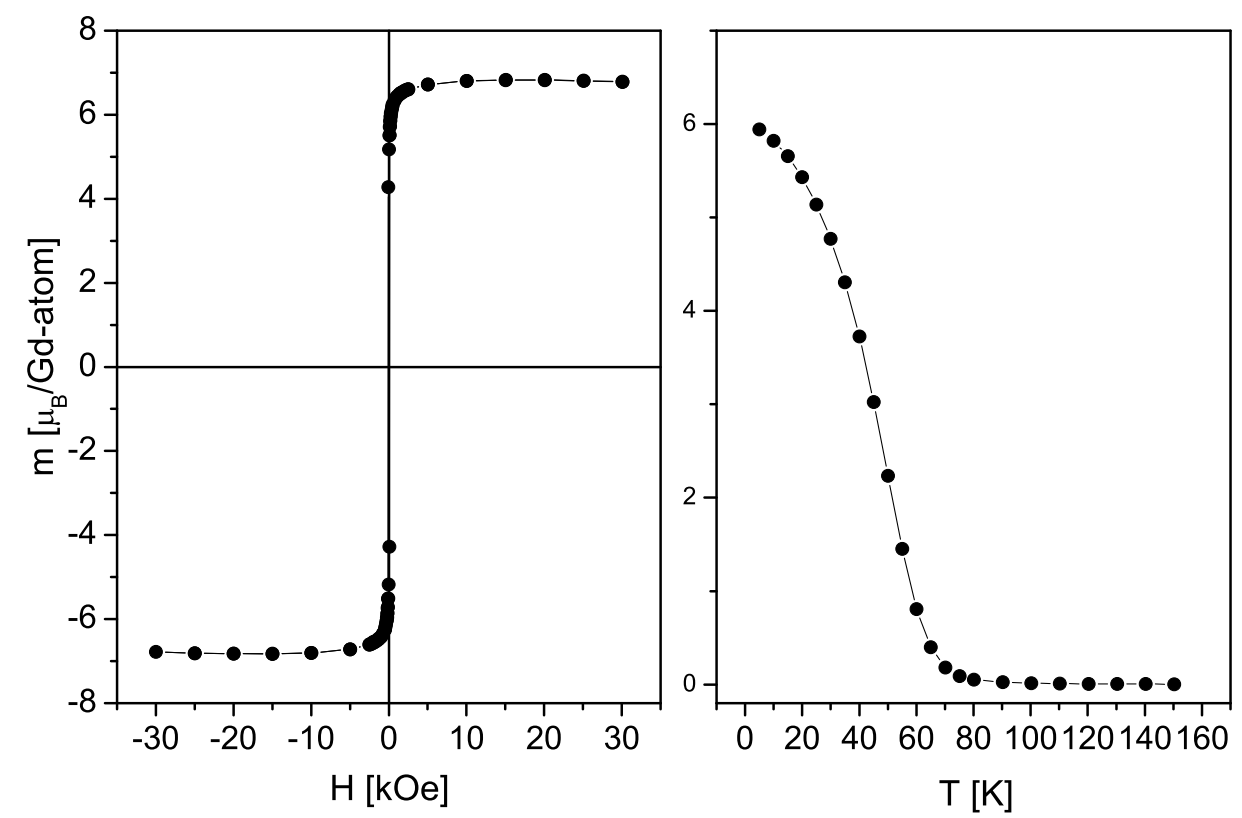

Figure 4.9: Hysteresis of a $500 \AA$ GdN film at $\mathrm{T}=5 \mathrm{~K}$ (left panel) and the magnetic moment per Gd ion vs. temperature in an external field of $200 \mathrm{Oe}$.

agreement with the bulk value determined from GdN powder samples by Li et al. [29]. The critical exponents are determined to $\beta=0.5$ and $\gamma=1.2$ and are consistent with the values expected from the mean field model.

The saturation moment reported by $\mathrm{Li}$ et al. is $6.84 \mu_{B} / \mathrm{Gd}^{3+}$ which is approximately $2 \%$ below the theoretical value of $7.0 \mu_{B} / \mathrm{Gd}^{3+}$. In $[41,35]$ it was stated that in $\mathrm{GdN}$ the magnatic moments of the $5 \mathrm{~d}$ electrons of the Gd couple antiferromagnetically to the magnetic moments of the N 2p electrons and compensate each other. This is in agreement with the saturation value found by $\mathrm{Li}$, since metallic Gd should have $7.5 \mu_{B}$ /atom essentially due to the magnetic moment of the $5 \mathrm{~d}$. The saturation moments of the GdN thin films were determined by SQUID magnetometry, the film thickness and the density were determined by x-ray diffraction and the sample area with a microscope. On this basis the number of Gd atoms are calculated and the magnetic moment measured by the SQUID was rescaled to $\mu_{B}$ /atom. In Fig. 4.9 a hysteresis curve of a $500 \AA$ film at $\mathrm{T}=5 \mathrm{~K}$ (left panel) and the magnetic moment per Gd ion in an external field of 200Oe vs. temperature (right panel) are shown. The hysteresis shows a soft ferromagnetic film with 
a coercitivity of $75 \mathrm{Oe}$ and a saturation field of $5 \mathrm{kOe}$. The saturation moment is $6.8 \mu_{B} / \mathrm{Gd}$ ion and confirms the value found by Li very well. In the left panel the temperature dependence of the film shows the expected spontaneous magnetization of a ferromagnet. The magnetic properties of GdN are apparently the best proof of the quality of the films. Wachter and Kaldis have examined the effect of oxygen impurities in GdN crystals and found that the saturation field of $\mathrm{GdN}_{0.96} \mathrm{O}_{0.04}$ is above 50kOe [27] and is shifted to higher fields with increasing oxygen content. The magnetization vs. temperature in an external field of $0.2 \mathrm{kOe}$ of the same $\mathrm{GdN}_{0.96} \mathrm{O}_{0.04}$ sample was below $0.2 \mu_{B} / \mathrm{Gd}$ ion at low temperature. In Fig. 4.9 the $\mathrm{GdN}$ film has a magnetization of about $6 \mu_{B}$ in the same external field at low temperatures. Although the crystal structure of the samples is only polycrystalline and the determination of the nitrogen content has an error of about $3-5 \%$ the overall picture testifies a very good sample quality with excellent magnetic properties and no evidence of impurities.

In [35] the band structure calculation states GdN as a semiconductor even at $T=0$. But the author mentioned that the band gap may be lower and the spin splitting could close the gap below $T_{C}$ for one spin channel and the material becomes a half metal. In this case one would expect a change in the resistance as the material changes from a semiconductor to a conductor. In $\mathrm{EuO}$ a comparable situation leads to a drop in the resistance of several orders of magnitude. A detailed article about the resistivity of EuO was published by Saphira and Foner [26]. The resistivity of the examined EuO crystals shows a transition at $T_{C} \approx 70 K$, but the drop varies between $2-10$ order of magnitude. The variation is explained by the amount of impurities. The samples are classified as thermally activated and nonactivated. For the activated samples the resistivity increases with decreasing temperature above $T_{C}$ as expected for a semiconducting material. The activation energy in the $\mathrm{EuO}$ samples found by Shapira was about $0.3 \mathrm{eV}$. The nonactivated samples have an almost constant resistivity in that range and the change of upon crossing $T_{C}$ is smaller. In Fig. 4.10 (left panel) the temperature dependence of the resistance of a GdN thin film is shown for different external magnetic fields. One clearly observes an increase of the resistivity with decreasing temperature. At $T_{C}$ the characteristic changes and the resistivity drops with 


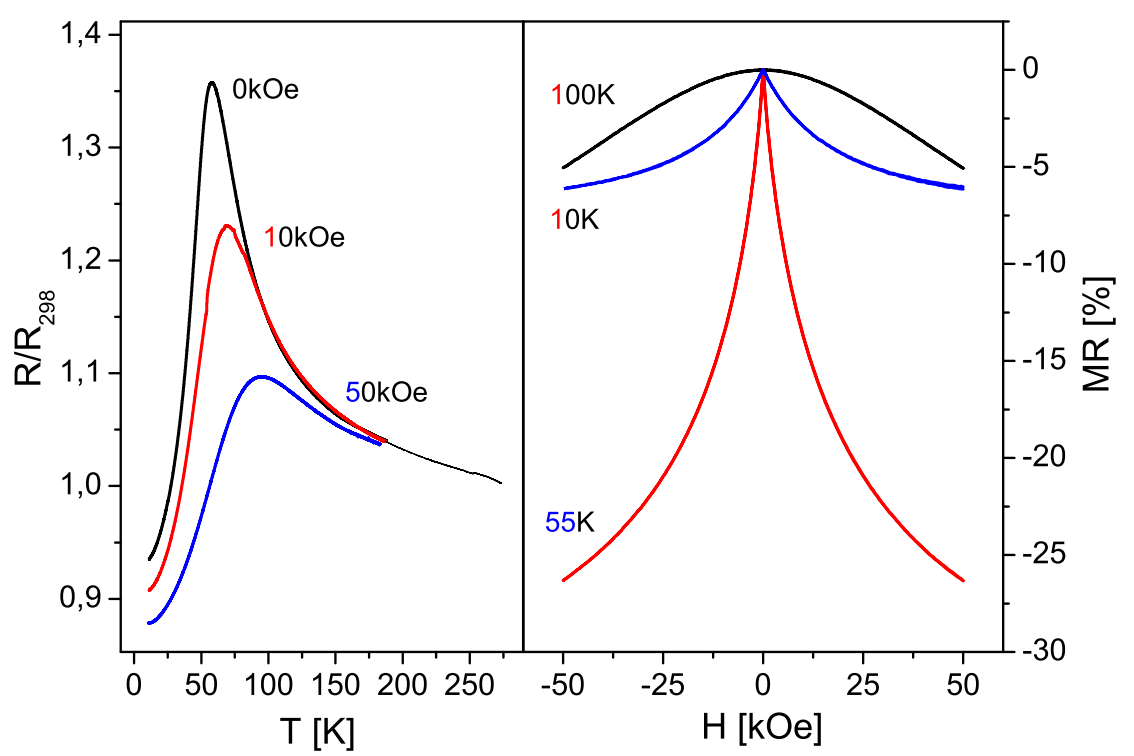

Figure 4.10: Resistance vs. temperature (normalized to the room temperature value) in different external fields of a $1000 \AA$ GdN film (left panel). The GdN films show a thermally activated conductance above $T_{C}$ and transition to a metal-like behavior at $T_{C}$. In the right panel the magnetoresistance below, above and near $T_{C}$ is shown $\left(\left(R_{50 k O e}-R_{0}\right) / R_{0}\right)$.

decreasing temperature like in a metal. As in $\mathrm{EuO}$ one has a semiconductorto-metal transition at $T_{C}$. The the effect is considerablly smaller than in the EuO crystals examined by Shapira et al. , but since the GdN films are polycrystalline one has to take into account that the amount of defect impurities can alter the transport properties of a semiconductor strongly. However, the thin films show a clear thermally activated conductance as expected for a semiconducting material but with an activation energy of less than $0.05 \mathrm{eV}$. The large resistivity drop in EuO was explained by Steeneken et al. by an exchange splitting of the conduction band in the ferromagnetic state that was proved with spin polarized XAS [42]. They attribute this splitting to a direct exchange interaction of the localized $4 \mathrm{f}$ moments and the delocalized $5 \mathrm{~d}-6 \mathrm{~s}$ conduction band states. The drop in resistivity would be a consequence of impurity or defects states then, that are located slightly below the bottom of the conduction band. A higher amount of defect impurities and a lower activation energy lead to a higher amount of charge carries above $T_{C}$ and the change in resistance at the transition is lowered. The low activation energy 
is an indication of a larger concentration of defect impurities. Also grain boundaries could have a certain effect since the nominal stoichiometry at the boundaries can differ and the resistivity in the boundaries may be different. Especially when the grains are semiconducting above $T_{C}$ but the boundaries provide an additional current path, because the amount of defect impurities is higher in the grain boundaries, the overall resistivity of the film would be lowered, suppressing the maximum at $T_{C}$.

Lambrecht [35] proposes a redshift at $T_{C}$ since the spin splitting of the conduction band lowers (or closes) the gap when magnetic ordering occurs. The estimated splitting from their band structure calculation is $0.3 \mathrm{eV}$. A similar redshift was observed in $\mathrm{EuO}$ and $\mathrm{EuS}$ [25]. An external magnetic field can enhance the magnetic ordering of GdN near $T_{C}$ due to the large uf moments leading to a enhanced spin splitting. Therefore one expects a negative magnetoresistance around $T_{C}$. In an external field of $50 \mathrm{kOe}$ a decrease of the resistivity at $T_{C}$ of $26 \%$ can be observed in the GdN films (see Fig. 4.10 right panel) and the maximum of the transition is shifted to higher temperatures. Shapira et al. [26] found a magnetoresistance in $\mathrm{EuO}$ that has the same behavior as in the GdN films, where the resistivity peak decreases, becomes broader and is shifted to higher temperatures by increasing the external field. In the ferromagnetic temperature regime the magnetresistance has an exponential decay with the external field whereas above $T_{C}$ the characteristic changes to a quadratic dependence (black curve on the right panel). This is a phenomenom known from the manganites and is interpreted there as a field driven nonmetal-metal transition [43], too.

Besides an experimental verification of the ground state the electronic properties of the GdN films are consistent within the picture of Lambrecht and the observations of Shapira and one can conclude that GdN is a semiconductor with a transition to a ferromagnet like EuO. 


\subsection{XMCD study of GdN}

\subsubsection{XMCD of the Gd L-edges}

As discussed in the previous section the interplay of the Gd5d-6s, Gd4f and the N2p electron levels have a sustained effect on the magnetic and electronic properties of the pnictides. In [41] the induced spin moments of the band electrons in GdN is decomposed into moments on the Rare Earth ions and the pnictogen ions. For all calculated Gd pnictides the moments are small and opposite in sign canceling each other out. Thus the net magnetic moment is almost completely due to the localized $4 \mathrm{f}$ electrons explaining the saturation moment of about $7 \mu_{B} / \mathrm{Gd}$ ion found in the magnetic measurements. The polarization of the different electron states is accessible by XMCD experiments through the Gd L- and M-edges and the N K-edge. To our knowledge no XMCD data for GdN is available yet.

Although the ferromagnetic exchange in $\mathrm{GdN}$ is probably not completely understood, it is clear that a certain amount of charge carries in the conduction band is needed to achieve a ferromagnetic RKKY exchange. Since the bottom of the conduction band in $\mathrm{GdN}$ is formed mainly by the $5 \mathrm{~d}$ electrons of the Gd it would be desirable to measure the polarization of these states, which can be done by measuring the magnetic dichroism of the Gd L-edge transition $(2 \mathrm{p} \rightarrow 5 \mathrm{~d})$. As mentioned in chapter 3 the theoretical description of the L-edges of the $\mathrm{RE}$ is a serious problem due to the intra-atomic dfCoulomb interactions. For Gd, contrary to expectations from the sum rules, the XMCD has the wrong sign for both edges and it is not possible to calculate a magnetic moment for the $5 \mathrm{~d}$ electrons from the sum rules. The Gd L-edge XMCD measurements at the GdN thin films were made at the dispersive beamline D11 at LURE. The transmission geometry makes it necessary to grow the GdN on a Kapton foil at room temperature since the Kapton cannot be heated to $400^{\circ} \mathrm{C}$. The L-edge absorption spectra measured in transmission geometry of a $2000 \AA$ GdN film on kapton is show in Fig. 4.11 in the upper panel. The absorption of the edges is normalized to a ratio of $2 / 3$ to $1 / 3$ reflecting the degenerancy of the core sates $\left(2 \mathrm{p}_{3 / 2}, 2 \mathrm{p}_{1 / 2}\right)$. The red curve is a step function that was used to normalize the edges. The small os- 


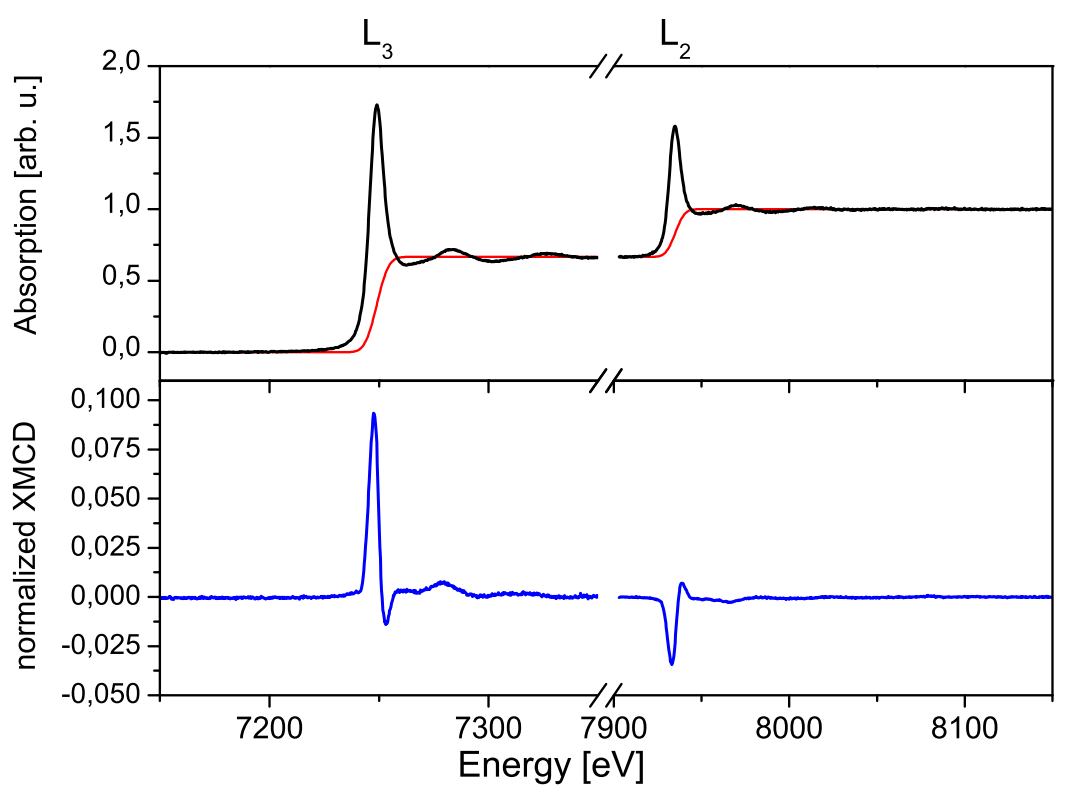

Figure 4.11: X-ray absorption at the $\mathrm{L}_{2}$ and $\mathrm{L}_{3}$-edge of $\mathrm{Gd}$ in $2000 \AA \mathrm{GdN}$ film on kapton (upper panel) and the normalized XMCD measured in an external field of $\pm 20 \mathrm{kOe}$ at $12 \mathrm{~K}$ (lower panel)

cillations at the end of the edges are EXAFS oscillations. In the lower panel the normalized XMCD of the Gd L-edges at low temperature is plotted (blue curve). A prominent anomaly found for the L-edge XMCD of the RE is the ratio between the $L_{3}$ and the $L_{2}$ XMCD. A large variation of the $L_{3} / L_{2}$ ratio over the Rare Earth series was reported by Baudelet et al. [22] and ascribed to the df-Coulomb interactions between the $4 \mathrm{f}$ and the $5 \mathrm{~d}$ electrons. A theoretical model to understand the branching ratio of the $\mathrm{L}_{2,3}$-edge XMCD was provided by van Veenendaal et al. [44]. For Gd or ferromagnetic Gd alloys with a half filled $4 \mathrm{f}$ shell and vanishing $4 \mathrm{f}$ orbital moment $\left\langle L_{z}\right\rangle=0$ always a 'correct' $\left|L_{3} / L_{2}\right|$ of 1 is observed. Here the $L_{2}$-edge XMCD is considrably smaller compared to the $\mathrm{L}_{3}$ part and the ratio is about 3 (see Fig. 4.11). Considering the symmetry of the probed $5 \mathrm{~d}$ states by the L-edges the ferromagnetic exchange in the $\mathrm{GdN}$ is attributed to the $5 \mathrm{~d} t_{2 g}$ manifolds which have a stronger $\mathrm{j}=3 / 2$ character [45], whereas the $5 \mathrm{~d} e_{g}$ are more of $j=5 / 2$ (chapter 4.1). The $\mathrm{L}_{3} \mathrm{XMCD}$ probes the final state polarization of the $5 \mathrm{~d}_{5 / 2}$ electrons and is therefore more sensitive to the $e_{g}$ states. Consequently the $\mathrm{L}_{2}$ XMCD, which probes the $5 \mathrm{~d}_{3 / 2}$ is sensitive to the polarization of the $t_{2 g}$ 


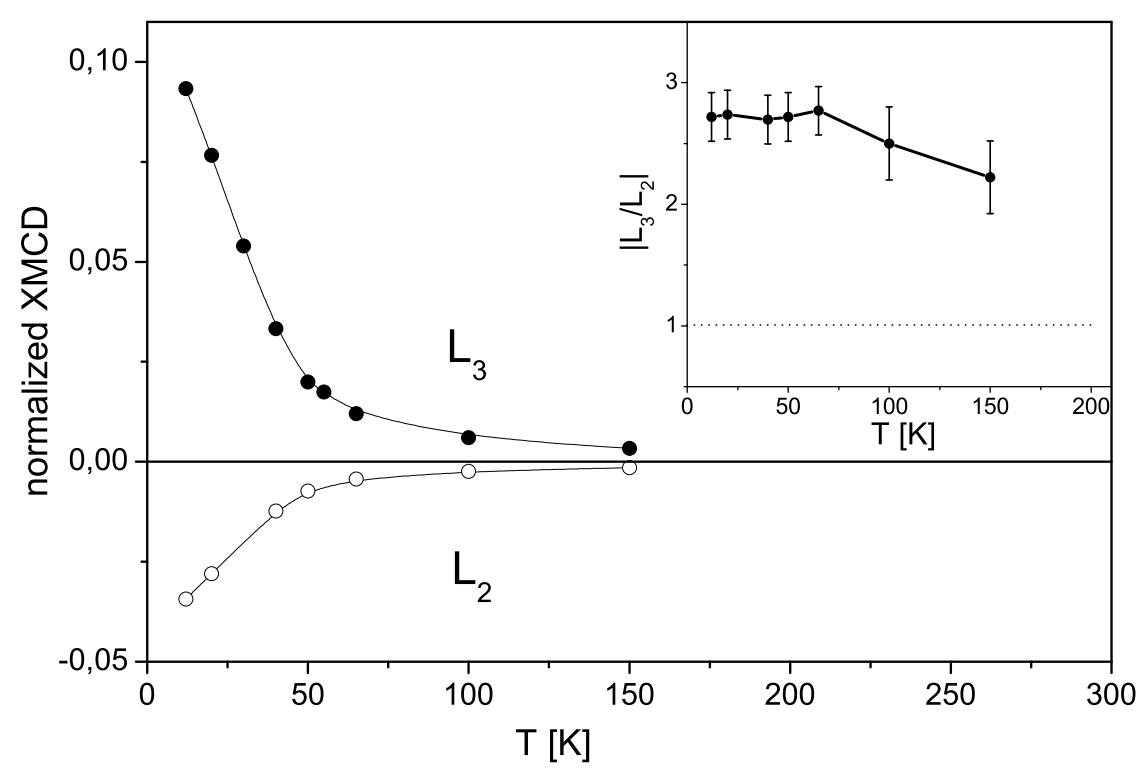

Figure 4.12: Temperature dependence of the normalized XMCD amplitude at the Gd L-edges of a $2000 \AA \mathrm{GdN}$ film on kapton. In the inset the $\left|L_{3} / L_{2}\right|$ ratio is plotted for the measured temperatures.

states. Remembering the structural differences for the room temperature prepared films (chapter 4.3) one can give an explanation within this picture. The increased lattice constant for room temperature grown samples decreases strongly the ferromagnetic exchange $J_{1}$ that is connected to the $t_{2 g}$ states and one would expect a decreased $t_{2 g}$ polarization and consequently a smaller $\mathrm{L}_{2}$-edge XMCD. Since the antiferromagnetic exchange is less sensitive to the increased lattice parameter $[34,46]$ the polarization of the $e_{g}$ states decreases less and the $\mathrm{L}_{3}$-edge XMCD as well. This results in a higher value of the $\left|L_{3} / L_{2}\right| \mathrm{XMCD}$ ratio as observed here. The temperature dependence of the L-edge XMCD in Fig. 4.12 shows a broad transition around $T_{C}$ which is caused by the external field of $20 \mathrm{kOe}$. The signal at $T_{C}$ is small compared to a sample grown at hight temperature, indicating that the $T_{C}$ of the GdN film is noticeably reduced. The unusual branching ratio of the L-edges shown in the inset is constant for temperatures below $T_{C}$ of bulk GdN and seems to drop for temperatures above $T_{C}$.

The variation of the macroscopic magnetization with temperature measured in a external field of $20 \mathrm{kOe}$ with a SQUID magnetometer is exactly 


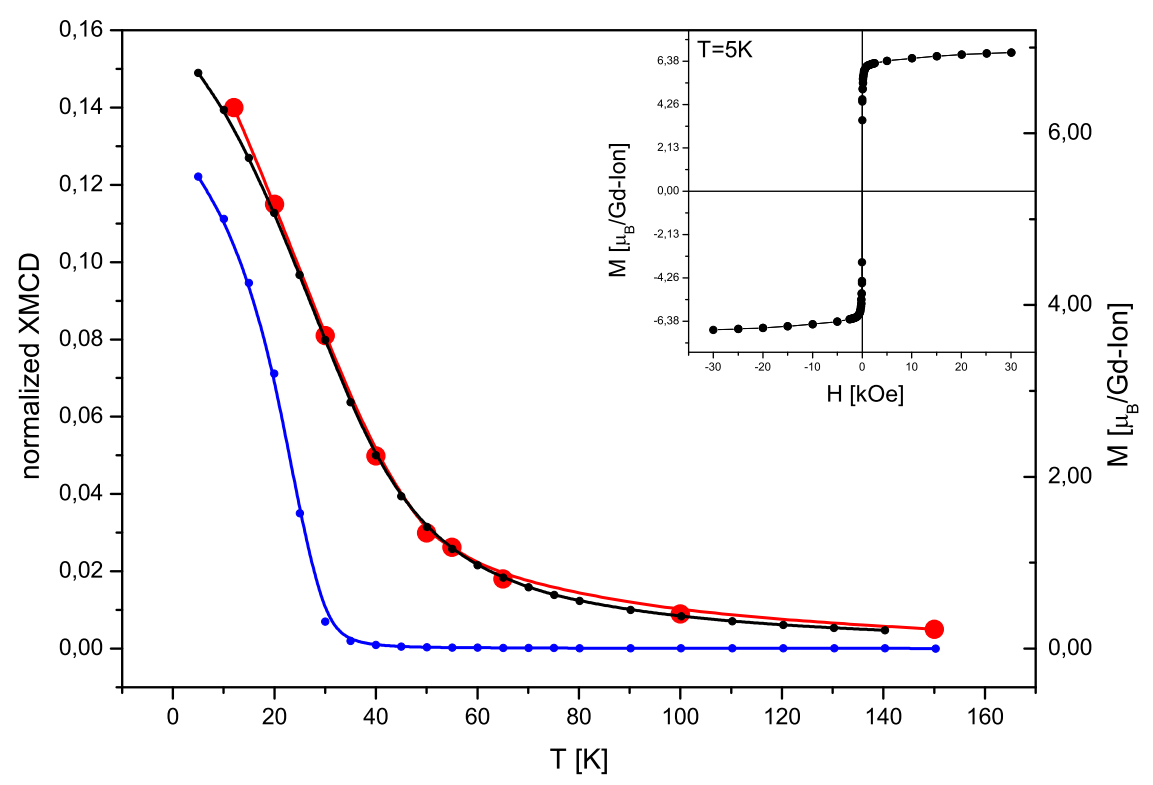

Figure 4.13: The magnetic moment and the $\mathrm{L}_{3}$-edge XMCD amplitude (red curve) of a $2000 \AA$ GdN film on kapton have the same temperature dependence in an external field of $20 \mathrm{kOe}$. The hysteresis curve in the inset shows clearly ferromagnetism.

the same as for the $\mathrm{L}_{3} \mathrm{XMCD}$ amplitude (Fig. 4.13). In a lower external field of 200 Oe the sample shows the expected reduced $T_{C}$ due to the growth at room temperature with a magnetization of $6 \mu_{B} / \mathrm{Gd}$ at $5 \mathrm{~K}$ (blue curve). The ferromagnetism with the expected saturation magnetization of $7 \mu_{B} / \mathrm{Gd}$ of the sample is confirmed by the hysteresis curve at $5 \mathrm{~K}$ shown in the inset. Although the origin of the increased lattice constant for the samples grown at room temperature is not known exactly the influence on the magnetic properties are consistent with the description of a ferromagnetic exchange $J_{1}$ via the $t_{2 g}$ states and a antiferromagnetic exchange $J_{2}$ via the $e_{g}$ states. Moreover, the sensitivity of the L-edge XMCD to the orbital symmetry of the probed final states provides the possibility to observe the change of the ratio between $J_{1}$ and $J_{2}$ directly.

The redshift predicted by Lambrecht lowers the absorption onset by about $0.3 \mathrm{eV}$ and should be visible in optical absorption measurements. In principal 


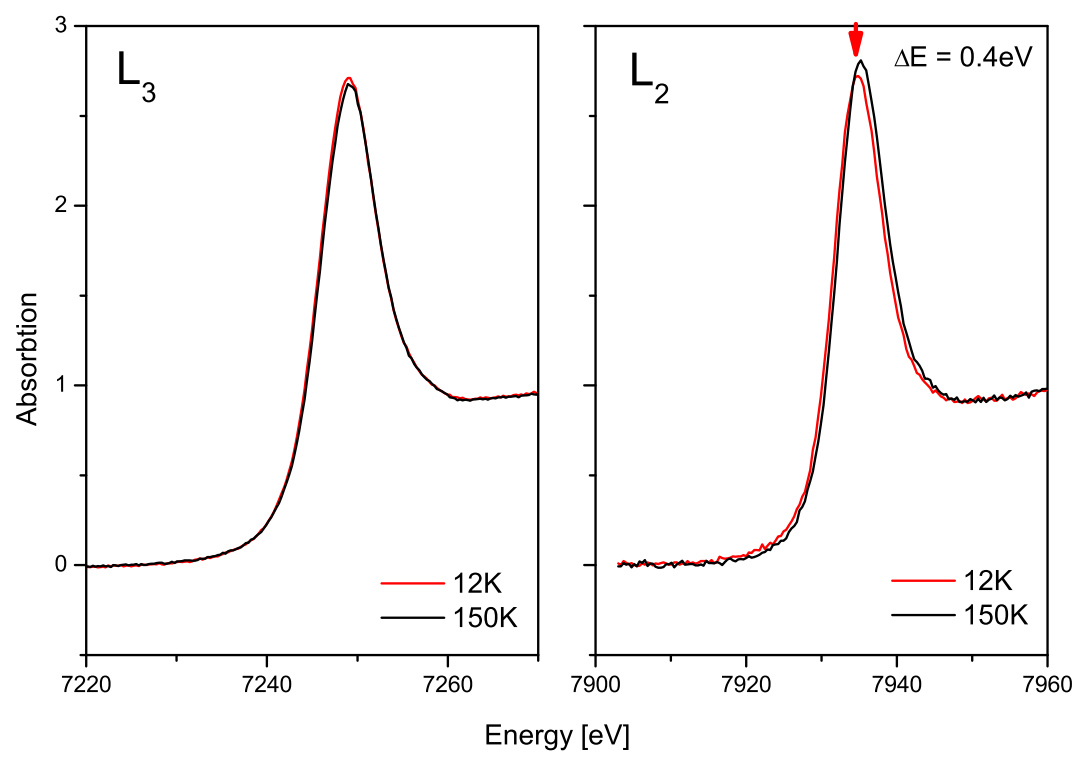

Figure 4.14: L-edge absorption spectra compared at high (150K) and low (12K) temperature for the $\mathrm{L}_{3}$ - and the $\mathrm{L}_{2}$-edge. In $\mathrm{L}_{3}$ spectra almost no change can be seen, whereas in the $\mathrm{L}_{2}$ a small shift to lower energy is observed

the redshift should lower the L-edge absorption spectra about $0.3 \mathrm{eV}$, which is low compared to the excitation energy of $7 \mathrm{keV}$. A relative comparison of the absorption spectra at $T>T_{C}$ and $T<T_{C}$ at the L-edges shows a small effect at the $\mathrm{L}_{2}$ and almost no effect at the $\mathrm{L}_{3}$-edge. The shift in the $\mathrm{L}_{2}$ edge spectra is about $0.4-0.5 \mathrm{eV}$. This in the range expected by Lambrecht. Again the $\mathrm{L}_{2}$-edge shows a response to the ferromagnetic ordering, whereas the $\mathrm{L}_{3}$-edge seems to stay passive. If the shift at the $\mathrm{L}_{2}$-edge is connected to ferromagnetic ordering in the GdN one would expect a similar temperature dependence like the XMCD. In order to give an estimation about the temperature dependence of the L-edge absorption onset the absorption edges taken at different temperatures were fitted with a Lorentzian peak. In Fig. 4.15 the deviation from the peak center at $150 \mathrm{~K}$ is plotted for the $\mathrm{L}_{2}$ and the $\mathrm{L}_{3}$-edges. The error bars specify the error of the peak center from the fit. The $\mathrm{L}_{3}$-edge location varies only little with temperature and the statistical error of the peak location is within $0.1 \mathrm{eV}$. From this result one can conclude that the $\mathrm{L}_{3}$ absorption onset in constant with respect to temperature or its change is below $0.1 \mathrm{eV}$. Consequently the accuracy of the determined peak 


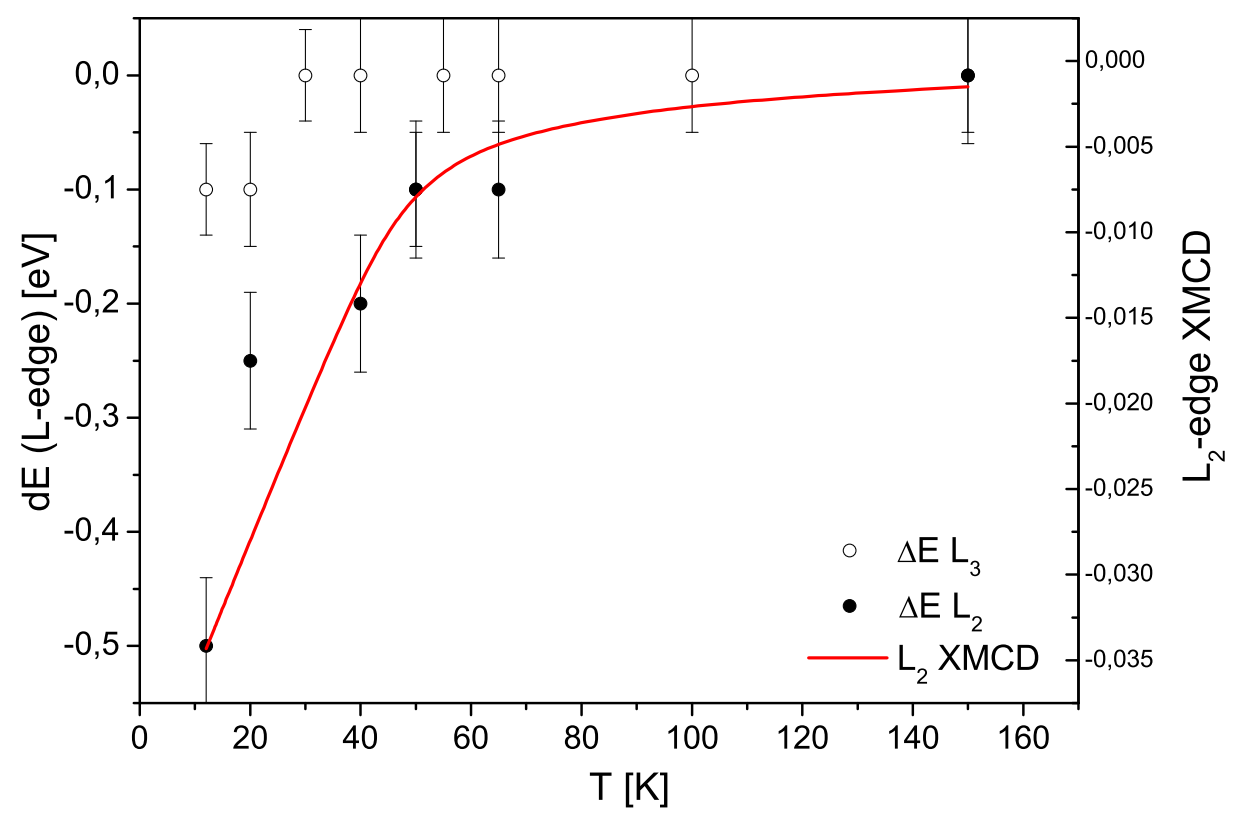

Figure 4.15: Deviation of the peak location of the $\mathrm{L}_{2}$-edge (full circles) and $\mathrm{L}_{3}$ edges (open circles) absorption spectra from the value at $T=150 \mathrm{~K}$. The red line marks the temperature dependence of the $\mathrm{L}_{2}$-edge XMCD amplitude.

location is also about $0.1 \mathrm{eV}$. In contrast to the $\mathrm{L}_{3}$ the $\mathrm{L}_{2}$-edge location shows as clear drop below $T_{C}$ of about $0.5 \mathrm{eV}$ and the temperature dependence of the absorption onset follows the $\mathrm{L}_{2}$-edge XMCD amplitude. Within the symmetry consideration from the $\left|L_{3} / L_{2}\right| \mathrm{XMCD}$ ratio the $\mathrm{L}_{3}$-edge absorption is connected to the antiferromagnetic $J_{2}$ and the $\mathrm{L}_{2}$ to the ferromagnetic $J_{1}$. The spin splitting in the band structure and the dedicated redshift is an effect of ferromagnetic ordering and this is reflected in a small shift of the $\mathrm{L}_{2}$-edge. 


\subsubsection{Nitrogen K-edge dichroism}

The polarization of the nitrogen $2 \mathrm{p}$ states is expected to be small in case of GdN $[41,36]$. Hence the K-edge XMCD effect is small as well compared to the L-edges and the detection of a XMCD signal at the nitrogen K-edge is a challenge. The experiments were carried out on samples grown at $450^{\circ} \mathrm{C}$ on $\mathrm{Si}(100)$. In this case the necessary cap layer for the GdN complicates the situation further, since a transmission experiment is not applicable due to the large absorption in the energy range of $400 \mathrm{eV}$. The experiment was therefore made in Total Electron Yield (TEY) at the beamline PM3 of the BESSY storage ring. Due to the cap layer the TEY measurements are not without complications. Preliminary experiments with different cap layers $(\mathrm{Al}, \mathrm{Cr})$ revealed that at least a $50 \AA \mathrm{Al}$ cap is needed to protect a $\mathrm{GdN}$ film over several weeks. The information depth of TEY in the energy range of the $\mathrm{N} \mathrm{K}$-edge is just around $50 \AA$ and thus an $\mathrm{Al}$ cap of that thickness is to thick. Therefore the GdN films were capped with $30 \AA$ thick $\mathrm{Cr}$ film and stored in a evacuated desiccator after preparation. At the PM3 the sample was transfered to the UHV chamber and absorption spectra were recorded at the N-K edge.

In the N K-edge spectra additional difficulties appear due to possibly adsorbed molecular nitrogen from the residual gas and due to x-ray absorption of higher harmonics from the grating monochromator. The higher harmonics lead to small but recognizable contributions of 2 nd and 3nd order radiation. Setting the monochromator to a 1st order energy of 400eV (N K-edge), a small 3rd order contribution with an energy of $1200 \mathrm{eV}$ is contained in the monochromated x-ray beam. This is about the energy where the $\mathrm{Gd} \mathrm{M}_{45}$ absorption edges are located. Although the damping of the 3rd order radiation by the monochromator is high the relative strong absorption of the $\mathrm{Gd}$ M-edges and the comparable small N-K edge absorption lead to an observable M-edge contribution in the N-K XAS. The small peak located in the pre-edge region at $395 \mathrm{eV}$ turned out to belong to the Gd M-edge absorption. In the left panel of Fig. 4.16 the peak is compared with a $\mathrm{Gd} \mathrm{M}_{4,5}$-edge XAS spectrum that was scaled down in energy by a factor of 3 (blue curve). In the inset the exact match of the $\mathrm{M}_{5}$-edge XAS and the peak in the $\mathrm{N}$ K-edge 


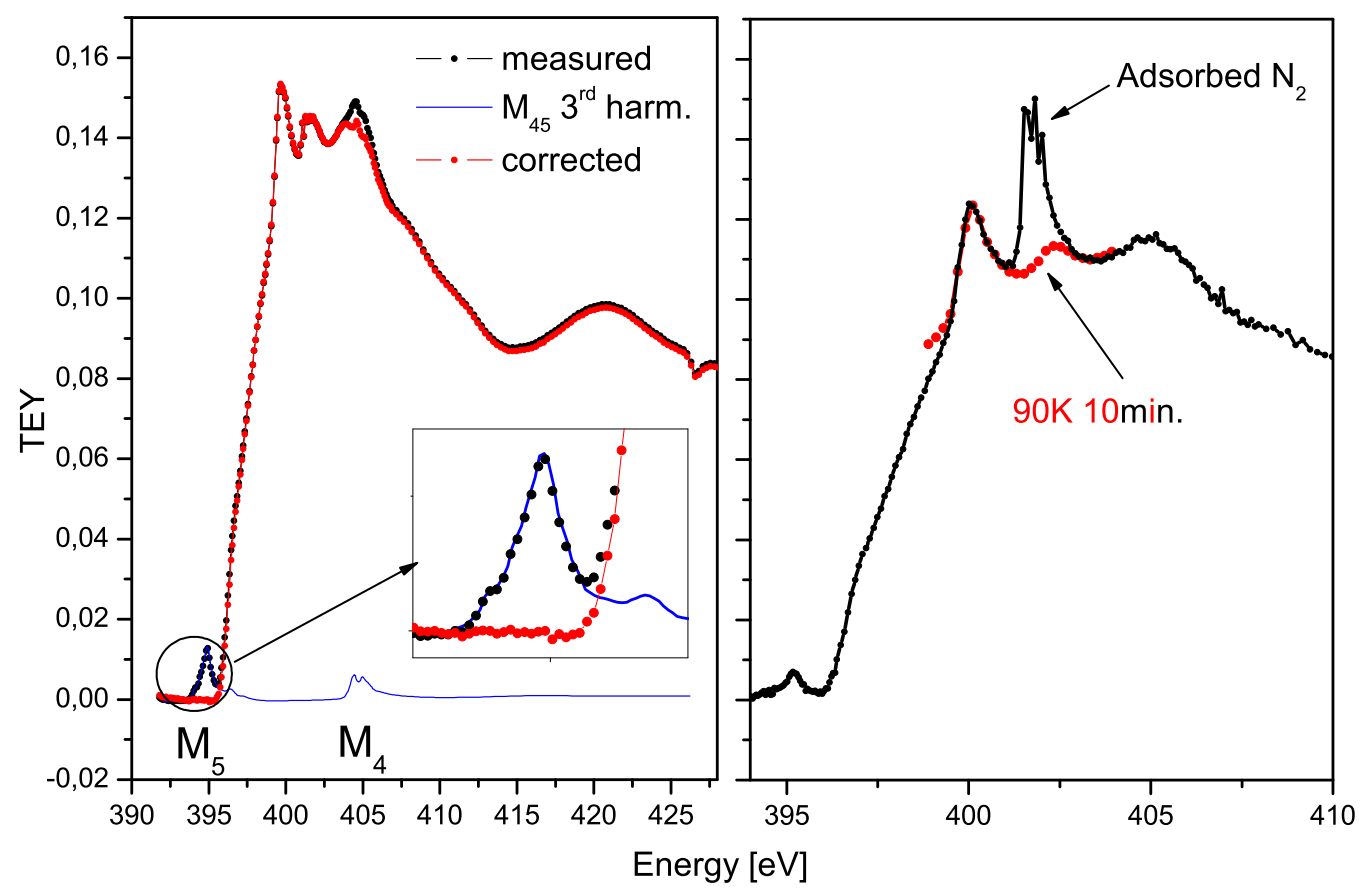

Figure 4.16: N K-edge XAS of a sputtered GdN film on Si with a perturbation of the Gd M-edge caused by the monochromator (left panel) and the effect of adsorbed molecular nitrogen at low temperatures (right panel). The blue curve in the inset is the $3 \mathrm{rd}$ order perturbation of the $\mathrm{Gd} \mathrm{M}_{45}$-edge absorption.

XAS can be seen. The $\mathrm{M}_{4,5}$ spectrum was fitted into the peak and the $\mathrm{N}$ K-edge XAS was corrected with the fitted $\mathrm{M}_{4,5}$ (red curve).

A second problem arises from the adsorption of molecular nitrogen at the sample surface, when the sample is cooled down to low temperature in a residual gas pressure above $10^{-9} \mathrm{mbar}$. In general the pressure in the sample chamber was always well below $5 \cdot 10^{-10} \mathrm{mbar}$, but on one occasion shortly after a sample transfer the pressure was in the range of $10^{-9} \mathrm{mbar}$. The adsorbed nitrogen XAS is shown in the right panel of Fig. 4.16 after the sample was transfered into the cryostat and cooled down to $20 \mathrm{~K}$. The adsorbed nitrogen could be easily removed by heating the sample to $90 \mathrm{~K}$ for 10 minutes (red curve) in proper UHV conditions.

Since the TEY of the N K-edge is very low in intensity the Cr cap was thinned with a Ar sputter gun. Although the beam current of the gun was low and the incident angle of the ion beam was small this procedure can 


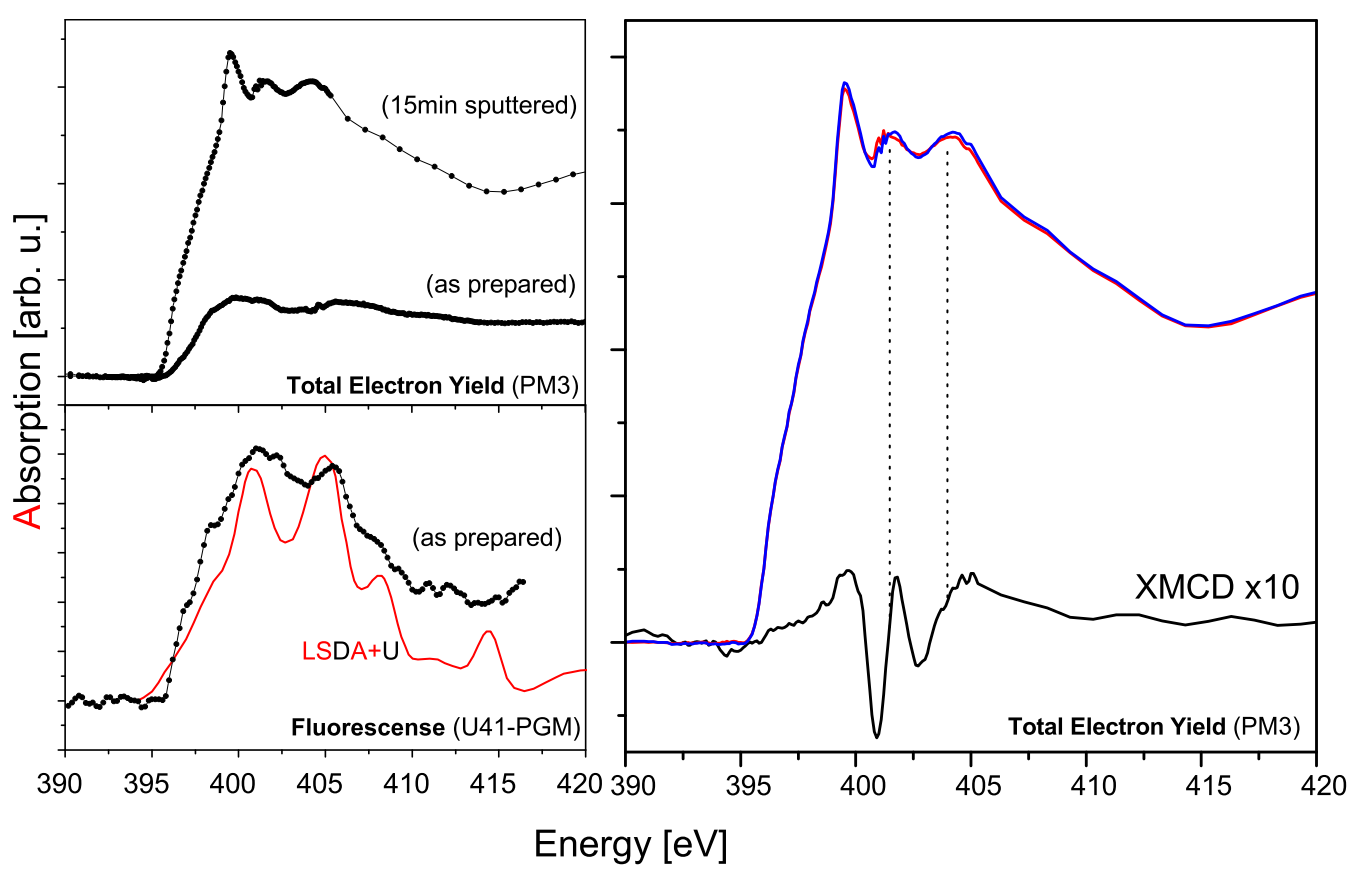

Figure 4.17: XAS of the as prepared and sputtered GdN film on Si (100) with a $30 \AA \mathrm{Cr}$ cap in TEY mode (upper left) and a reference spectra in total fluorescence yield of an as prepared GdN film with a $50 \AA A$ cap (lower left). The XMCD of the $\mathrm{N} \mathrm{K}$-edge in magnetic field of $10 \mathrm{kOe}$ at $15 \mathrm{~K}$ is shown in the right panel

cause certain damage at the surface. A complete removal of the cap was not desired, because it was not possible to heat the sample in the UHV chamber and there was no way to regenerate the surface. Therefore the $\mathrm{Cr}$ XAS was monitored after every 5 minutes of sputtering. After 15 minutes the Cr peak intensity had dropped about 2/3 in height and a N K-edge XAS was recorded. In Fig. 4.17 the N K-edge XAS before and after the sputter process can be seen (upper left). The as prepared sample shows only a small XAS with two broad maxima. The thinning of the Cr cap enhances the XAS significantly and the spectra shows a fine structure with three peaks. The related XMCD at the $\mathrm{N} \mathrm{K}$-edge at $T=15 K$ is shown in the right panel. One observes a clear magnetic signature. The first peak in the N K-edge XAS spectra does not have a clear magnetic response. Comparing the as prepared and the sputtered spectra it is obvious that the first peak appears after the sample was sputtered. In spite of all care during the sputtering it is always possible that there is a certain amount of intermixing at the interface 
between $\mathrm{Cr}$ cap and GdN and the contribution at the beginning of the $\mathrm{N}$ K-edge may belong to nonmagnetic $\mathrm{CrN}$. To obtain a undisturbed N K-edge XAS a reference spectrum from an as prepared sample was recorded at the beamline U41-PGM in total fluorescence yield. A fluorescence spectrum has a higher information depth since the mean free path of photons in a solid is greater than for electrons. The measurement was enabled by the group of C. Hague during a spectrometer test at BESSY. XMCD measurements could not be carried out. In the fluorescence spectrum the ambiguous peak close to the edge is much smaller or even missing. It shows essentially two maxima, which are also indicated in the TEY spectrum of the as prepared sample. Together with the fact that there is almost no XMCD signal at this location the peak must be attributed to an effect induced by the sputter process. The red dotted line is a partial DOS spectrum calculated with LSDA+U by A. Perlov for GdN [47]. The calculation and the as prepared fluorescence spectra are in a good agreement. This encourages the assumption that the spectral weight of the $\mathrm{N} 2 \mathrm{p}$ states from the $\mathrm{GdN}$ is represented by the two main peaks observed in the fluorescence spectrum. The XMCD spectra in the right panel of Fig. 4.17 has a more complicated spectral response as usually observed from a K-edge XMCD in the magnetic transition metals. On a first sight the shape is superimposed by the two derivate-like signals generated by the two peaks of the XAS spectra marked by the dotted line.

The total electron yield spectra and the observed XMCD are an obviously challenge since the signals are very small and the problems with a cap layer are not easy to handle. But the comparison of the fluorescence spectrum and the calculated spectrum of Perlov confirm that besides the contribution at the beginning the XAS spectrum is resulting from GdN. The observed XMCD is therefore in agreement with the prediction by Petukhov [41] and Aerts [36] whereupon the $\mathrm{N}$ sites are magnetically polarized via the $5 \mathrm{~d}$ electrons. To ensure that the magnetic signal originates from the GdN elemental hysteresis curves were recorded by measuring the Gd M4-edge XMCD and the first peak in the N K-edge XMCD. Since the polarization at the PM3 cannot be switched between two helicities during such a measurement a hysteresis curve was measured using a method proposed by Goering [48]. One has to run a complete field loop at fixed energy on top of the XMCD peak and a second 


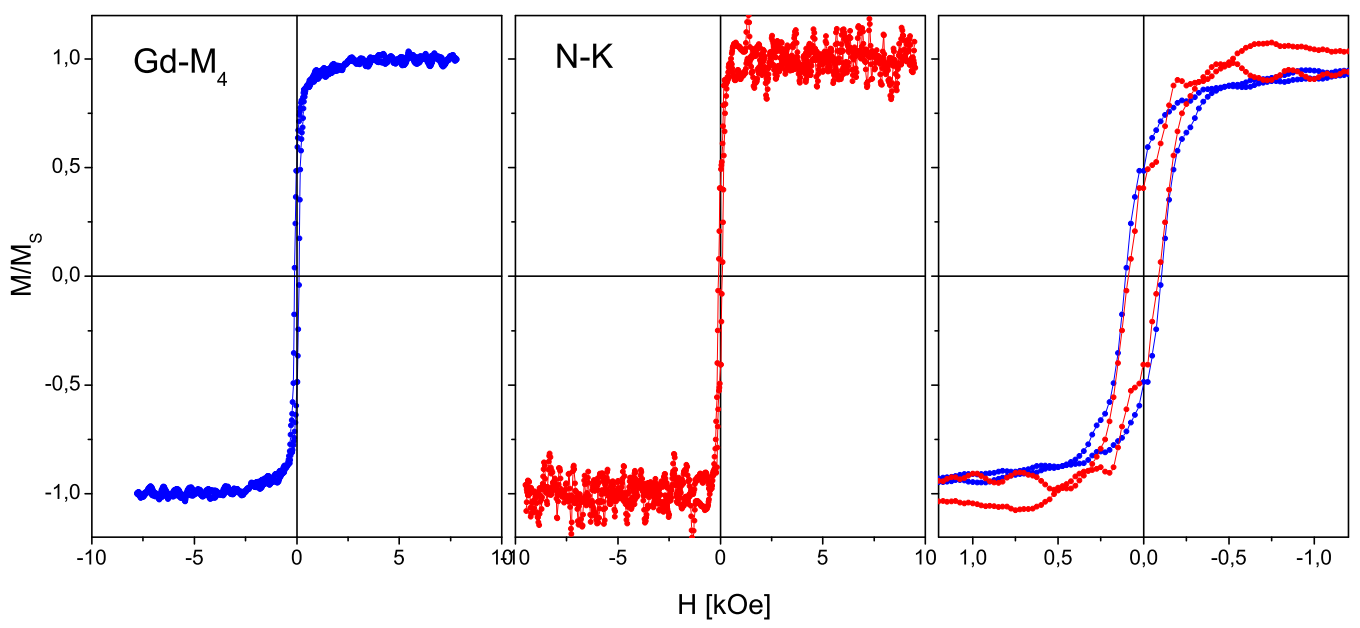

Figure 4.18: Elementla resolved hysteresis from the $\mathrm{Gd} \mathrm{M}_{4}$-edge XMCD (left panel) and the N K-edge XMCD(middle panel). The right panel shows the coercitivity of the $\mathrm{Gd} \mathrm{M}_{4}$ - and the N K-loop

loop at the nonmagnetic pre-edge. The field dependence of the TEY (which does not depend on the samples magnetization) is then corrected with the pre-edge loop. With this method one can only measure half a loop and the curves are always symmetric at the positive and negative field axis. In Fig. 4.18 the hysteresis loops of the $\mathrm{Gd} \mathrm{M}_{4}$-edge XMCD and the N K-edge XMCD are shown. In the right panel both loops are plotted together in a smaller magnetic field range. Due to the very small XMCD at the N K-edge the hysteresis loop from the nitrogen is more noisy but the loops match very well and confirm that the N K-edge XMCD has the same field dependence as the Gd M-edge and are therefore a proof that the measured N K-edge XMCD originates from the GdN.

The XMCD measurements of the GdN thin films clearly show that GdN is a ferromagnet, where the magnetic exchange is provided via the $5 \mathrm{~d}$ electrons. This is in agreement with the suggestion of Wachter et al. [27] that GdN needs a small amount of RKKY exchange coupled charge carries $(\approx 6 \%)$ in the conduction band to couple ferromagnetically. Also the polarization of the N2p states proposed by theory is observable with N K-edge XMCD, but a uniform picture of the electronic structure of Rare Earth pnictides does not exist up to now and the theoretical descriptions of GdN remain problematic 


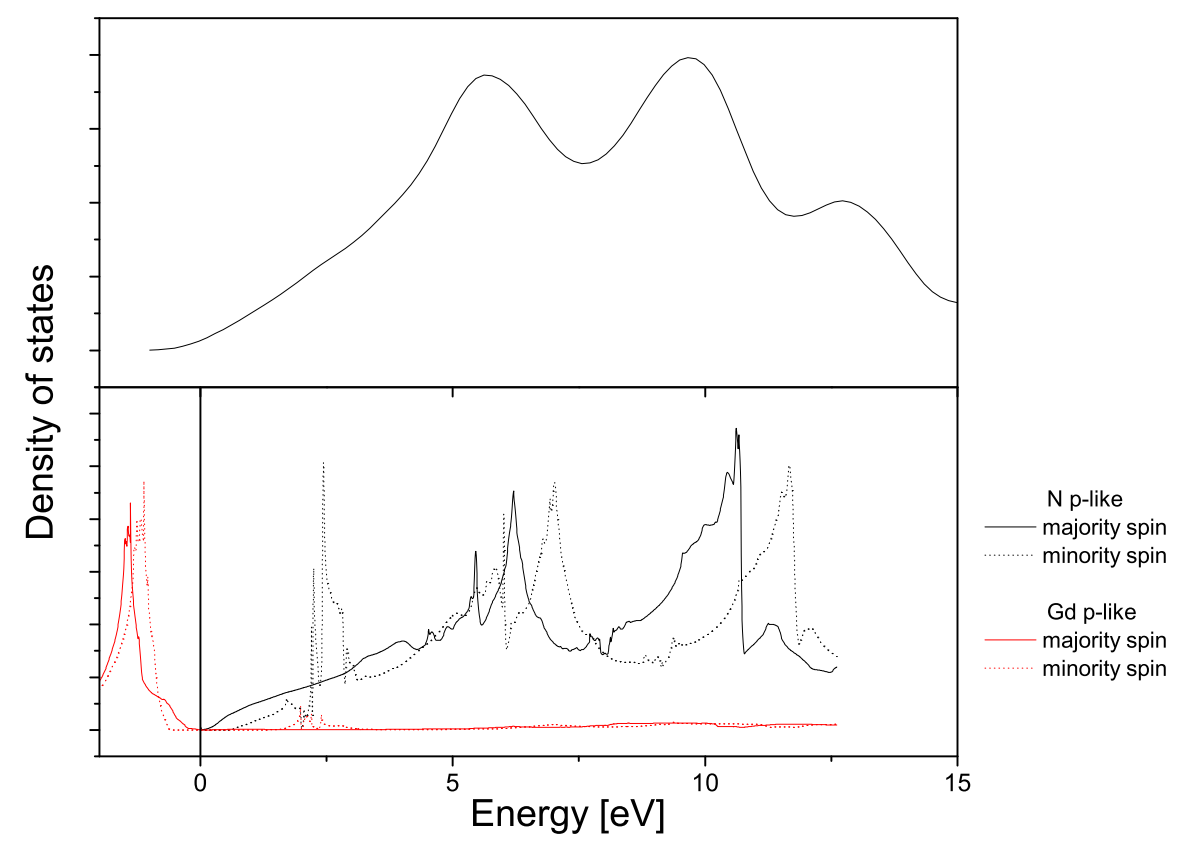

Figure 4.19: Calculated N K-edge partial DOS by Perlov (upper panel) and the spin resolved DOS with p-symmetry by Aerts.

due to the half open $4 \mathrm{f}$ shell. The approach by Aerts et al. inculding the 4f states leads to a half metallic groundstate [36], whereas the calculation of Perlov predicts a semiconducting groundstate [47]. In Fig. 4.19 the calculated N-K XAS (upper panel) and the N p-like DOS of Aerts (lower panel) have almost the same spectral weight between $5-10 \mathrm{eV}$. This is also in agreement with the fluorescence spectra of GdN (Fig. 4.17). The $4 \mathrm{f}$ correlations in the calculation of Aerts leads to a p-f hybridization of the empty states which is reflected by the peak around $2.5 \mathrm{eV}$. There is no clear evidence of this in the fluorescence spectrum. In the calculation of Aerts et al. the occupied If levels are located at $14.5 \mathrm{eV}$ below the Fermi level, which is very low regarding to the $4 \mathrm{f}$ level of $8 \mathrm{eV}$ below $E_{F}$ found in the XPS spectrum (see Fig. 4.5). The difference of the spin up and down contributions in the density of states in the calculation of Aerts is located in the same energy range as the N K-edge dichroism. Taking into account that a XAS spectra is in first order a probe of the density of states (including the lifetime broadening of the final state) the observed two peak structure fits into the the calculation of Aerts 
with respect to the energy location. The theoretical description of the Rare Earth nitrides remains a challenge and experimental spectroscopic data of high quality nitrides are essential to shed more light onto these compounds.

\subsection{Summary}

In this chapter the structural, electronic and magnetic properties of the GdN thin films were investigated. The reactive sputtering of GdN thin films leads to polycrystalline films with a rocksalt $(\mathrm{NaCl})$ structure. The substrate temperature has a large effect on the structure and the average lattice constant of the films decreases with increasing substrate temperature from $5.19 \AA$ at room temperature to $5.05 \AA$ at $450^{\circ} \mathrm{C}$. The stoichiometry is found to be $\mathrm{Gd}_{50} \mathrm{~N}_{50}$ within the accuracy of the here applied methods (RNRA, XPS). With in situ XPS spectra it was validated that the prepared GdN films are free of contaminations. The macroscopic magnetization of the films yields the expected Curie temperature of the bulk material $\left(T_{C, G d N}=59 \mathrm{~K}\right)$ and a saturation moment of $6.8 \mu_{B} / \mathrm{Gd}$ ion. Transport measurements show a semiconductor-to-metal transition as it is expected from theory. The effect is notably smaller than in the comparable system $\mathrm{EuO}$, which is explained by the higher amount of defect impurities due to the structure of the GdN films. The XAS and XMCD experiments made at the Gd L-edges and the N Kedge show a consistent picture within the theories of Lambrecht et al. [35,41] and Aerts et al. [36]. The polarization of the $\mathrm{Gd} 5 \mathrm{~d}$ and the $\mathrm{N} 2 \mathrm{p}$ electron states is clearly visible. From the Gd L-edges it was found that the Gd $5 \mathrm{~d}$ polarization reflects the temperature dependence of the macroscopic magnetization that is dominated by the Gd-4f electrons. The elemental resolved hysteresis curves state the connection between the $\mathrm{Gd}$ 4f magnetic moment and the N 2p electrons via the Gd-5d electrons. Also the redshift predicted by Lambrecht [35] is observable in a small shift of the $\mathrm{L}_{2}$ absorption onset. 


\section{Chapter 5}

\section{GdN/Fe multilayers}

\subsection{Magnetism in RE transition metal inter- metallics and multilayers}

When two magnetic materials are combined in an alloy or a multilayer system a key point is how the magnetic coupling takes place at the interface. A successful description of the coupling mechanism in intermetallic compounds of rare earth and transition metals based on the $3 \mathrm{~d} 5 \mathrm{~d}$ hybridization was given by Johansson et al. $[49,50,51]$ and can be seen as the basic principle. A brief description of the coupling mechanism is given in the following part of this section. Considering the energy levels of a free atom the transition-metal $3 \mathrm{~d}$ states are considerably lower lying in energy than the rare earth $5 \mathrm{~d}$ states. Forming a molecule between the two atoms lead to a mixing of the $3 \mathrm{~d}$ and $5 \mathrm{~d}$ levels where the bonding levels have mainly $3 \mathrm{~d}$ and the antibonding ones mainly $5 \mathrm{~d}$ character. The amount of mixing depends on the energy distance between the $3 \mathrm{~d}$ and $5 \mathrm{~d}$ levels. In a solid the energy levels broaden to energy bands, which gives in a simplified picture 'bonding bands' with mainly 3d and 'antibonding bands' with 5d character. A schematic illustration is given in Figure 5.1 on the left side. In the case of a magnetic transition metal where the $3 \mathrm{~d}$ band is spin polarized the $3 \mathrm{~d}$ majority band is lowered in energy and the amount of $5 \mathrm{~d}$ character is decreased in the bonding band due to the greater distance in energy to the $5 \mathrm{~d}$ majority band. This asymmetry leads to different amount of hybridization for the majority and minority bands as 


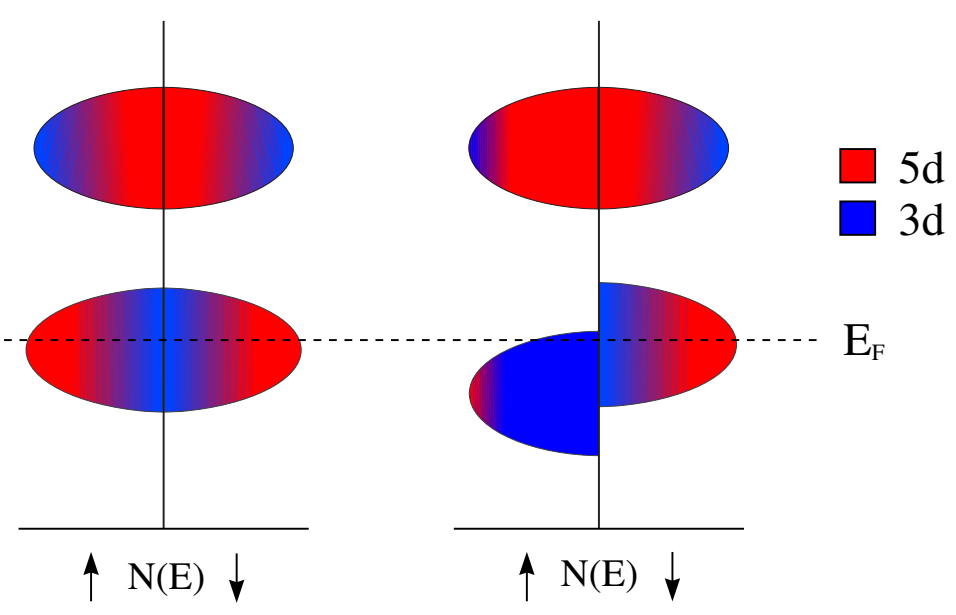

Figure 5.1: Schematic representation of the 3d5d-hybridization for unpolarized (left) and spinpolarized (right) metals

shown on the right side of Figure 5.1. In this case the spin up occupation of the $5 \mathrm{~d}$ part is lower than the spin down part leading to a total spin down $5 \mathrm{~d}$ moment on the rare earth atom. Consequently the $3 \mathrm{~d}$ and $5 \mathrm{~d}$ moments will be in opposite directions. For most rare earth metals the $4 \mathrm{f}$ electrons retain their localized atomic state and are therefore neglected in this picture. On the other hand the magnetic moment in these materials is dominated by the 4f electrons and the magnetic coupling mechanisms in rare earth transitionmetal intermetallics involve complex interaction between different electron systems. The $5 \mathrm{~d}$ electrons make only a small contribution to the magnetic moment but they act as the mediators between the well localized $4 \mathrm{f}$ shells, that do not have a direct overlap in a solid, and the itinerant $3 \mathrm{~d}$ electrons in the transition metal. It can be assumed that the same coupling mechanism takes place at the interface of a rare earth intermetallics multilayer system.

A popular system of a rare earth intermetallic multilayer is the Gd/Fe system that was subject of rich investigations in the 80's and 90's. Even though a lot of experimental and theoretical results has been published there are still open questions and the interest in this system persists until now $[52,53,54,55]$. In the $\mathrm{RE}$ the intra-atomic $4 \mathrm{f}-5 \mathrm{~d}$ coupling is always parallel and in case of Gd where the LS coupling is parallel too; this leads to an antiparallel coupled net magnetization and thus Gd/Fe multilayers always form an artificial ferrimagnet in their groundstate. The magnetic properties of such a multi- 
layer system depend strongly on the coupling strength at the interface and the layer thicknesses. A theoretical description of the magnetic phase dia-

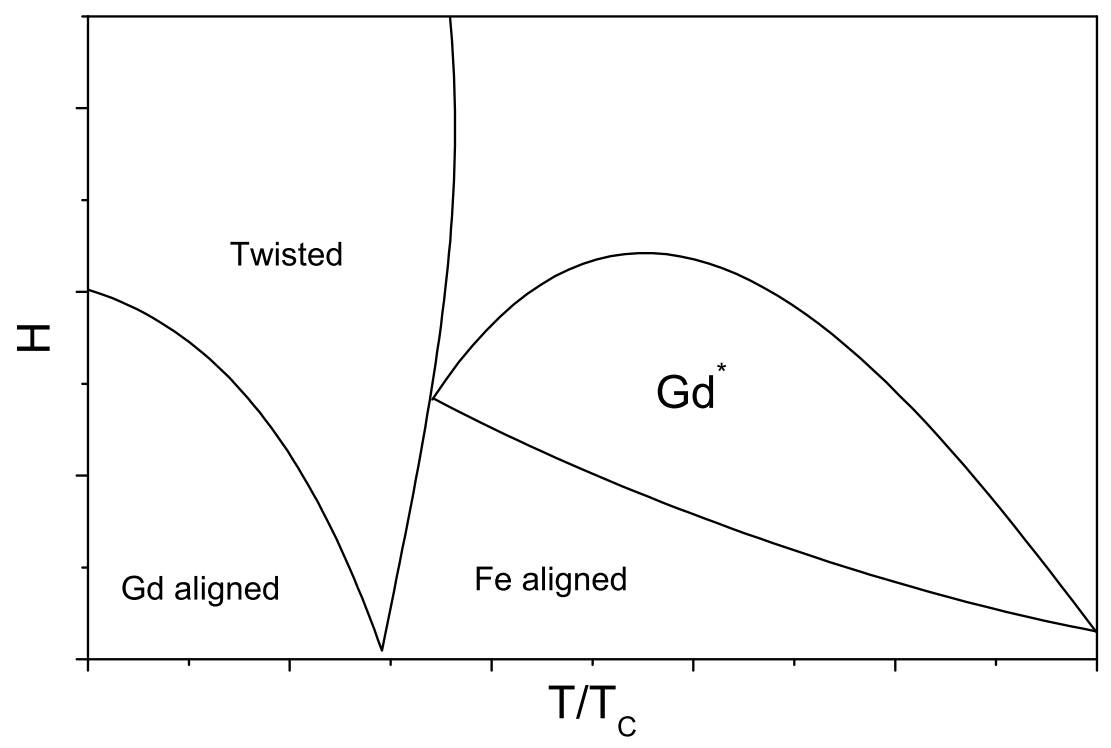

Figure 5.2: Phase diagram of a Gd/Fe system for weak coupling from [56]. $T_{C}$ is the Curie temperature of the iron. $\mathrm{Gd}^{\star}$ is a modified Fe aligned phase were the Gd layers between the aligned Fe interfaces are twisted.

gram of such a system was given by Camley and others $[57,56,58,59,60]$. The phase diagram (Fig. 5.2) is divided into three regions depending on temperature and the external magnetic field. In the Fe aligned state, the Fe layers magnetic moments are oriented parallel to the applied field and those of the Gd layers antiparallel. This phase is observed at high temperatures between $T_{C, G d}=292 \mathrm{~K}$ and $T_{C, F e}=1043 \mathrm{~K}$ when the $\mathrm{Gd}$ is in the paramagnetic state or when the Fe has the major net moment. Depending on the $\mathrm{Gd} / \mathrm{Fe}$ thickness ratio for $T<T_{C, G d}$ and low fields the Gd aligned state is observed where the situation is just the opposite way. At a certain temperature the magnetizations of the Gd and Fe layers have the same magnitude and cancel each other out. This is called the compensation point. Increasing the external field leads to the so called twisted state with a non collinear angular distribution between the Fe and the Gd layer magnetizations, because the system has to find a compromise to minimize the higher Zeeman energy in the applied field. The twist is determined by the 'stiffness' of the materials. Camley gives an estimation of the number of layers over 
which a $180^{\circ}$ turn of the magnetization will occur based on a simple model of the domain wall thickness from [61]. For Fe 62 layers and for Gd 22 layers are needed for a $180^{\circ}$ turn within this estimation. A numerical method to calculate the angular distribution of the magnetic moments in a given field was given by Motokawa et al. [62,63,64]. For antiferromagnetically coupled systems with a coupling strength like in Camley's description a twist at a given temperature occurs only if the external field exceeds a critical value $H_{C}$. Below $H_{C}$ the multilayer remains in the either Gd or Fe aligned state. This onset of the twist above $H_{C}$ is seen in hysteresis loops by a kink at higher fields marking an increase of the slope. In this interplay the phase diagram is determined by the thickness and the magnetic exchange constants inside and between the two materials. Experimental results related to the Gd/Fe system can be found in $[65,66,67,68,59]$. The area of the twisted phase in Fig. 5.2 is strongly influenced by the coupling strength $J_{I}$ at the interface. In [56] the phase diagram is calculated for 'strong' $\left(J_{I}=J_{F e}\right)$ and 'weak' coupling $\left(J_{I}=J_{G d}\right)$ at the interface. The main differences between the two cases is the onset of the twisted phase at low temperatures which is shifted to considerably lower fields for weak coupling and the occurrence of modified Fe aligned states $\left(\mathrm{Gd}^{\star}\right)$ at higher temperatures and higher fields. These states are Fe aligned states where the Gd magnetization is no more completely antiparallel aligned but the atomic layers in the middle of each Gd layer are rotated into the field direction.

\subsection{Sample preparation and structure}

The preparation of the multilayers was done by reactive ion beam sputtering from pure Fe and Gd targets with Argon. A detailed description of the entire process was given in chapter 2. For the multilayer systems all XMCD experiments were done in the transmission geometry, because in transmission all layers contribute to the magnetic signal giving a better sensitivity to interface effects. From the fact that the transmission at the absorption edge should come to at least $30 \%$ to get a sufficient threshold above the dark current of the photodiode arises the demand for a carefully prepared sample on a very thin substrate. In this work two different substrates were used. 
For the $\mathrm{Fe} \mathrm{L}_{2,3^{-}}$and the $\mathrm{Gd} \mathrm{M}_{4,5^{-}}$edges that are in the soft $\mathrm{x}$-ray regime $(h \nu<1500 \mathrm{eV})$ the absorption by the substrate is very high and the samples were prepared on thin Mylar foils $(d=1,5 \mu \mathrm{m})$ mounted on small copper frames (chapter 2). The foils were coated with a $100 \AA$ thick $\mathrm{Al}$ buffer to improve the thermal conductance and to achieve a better defined surface. The absorption of such a substrate is already $30 \%$, and the thickness of the multilayer is limited by the absorption of the Gd M-edges to $d<250 \AA$ to stay above the transmission limit. For the $\mathrm{Gd} \mathrm{L}_{2,3}$-edges that are located between 7 and $8 \mathrm{keV}$, the absorption from the substrate is much smaller and 7,5 $\mathrm{mm}$ Kapton foils could be used that are much easier to handle than the mylars. Also the absorption at the Gd L-edges is smaller and the total thickness of the multilayer was limited by the preparation time, which is determined by the average sputter rate $(\sim 0.4 \AA / \mathrm{s})$ and the fact when switching the preparation from GdN to Fe a delay of about 60 seconds was made to pump away the nitrogen. It was found that $4000 \AA$ in total thickness was a suitable dimension, giving a total process time of about 5 hours.

For every multilayer reference samples on Si (100) were prepared in the same process to check the crytallinity and the macroscopic magnetization. In contrast to the single GdN layers, which always grow polycrystalline, all multilayers were found to grow textured in a (100) orientation. In Figure 5.3 typical XRD patterns of three samples with different GdN thickness are shown. Besides the the intense Si (400) peak from the substrate two major peaks can be seen, at $34.7^{\circ}$ the $\operatorname{GdN}(200)$ and at $64.8^{\circ}$ the $\mathrm{Fe}(200)$ peak. This is unusual for Fe since the densest plane for bcc-Fe is (110), but due to the mismatch between the two materials they stabilize each other in the (100) orientation. A similar effect was found in our group for other rare earth transition metal multilayers $[69,70,71]$. Due to the small layer thickness the peaks are very broad and calculating the grain size using Scherrer's formula (2.2) one obtains the layer thickness. Referring to the position of the peaks we obtain a lattice constant of $0,287 \mathrm{~nm}$ for the Fe layers which is in very good agreement with the literature value. Calculating the lattice constant from the $\operatorname{GdN}(200)$ peak gives $0.517 \mathrm{~nm}$, which is significantly larger than one would expect from the results of the single layers or bulk GdN, where a value of $0.505 \mathrm{~nm}$ was found. With the applied $\Theta-2 \Theta$ geometry (Bragg- 


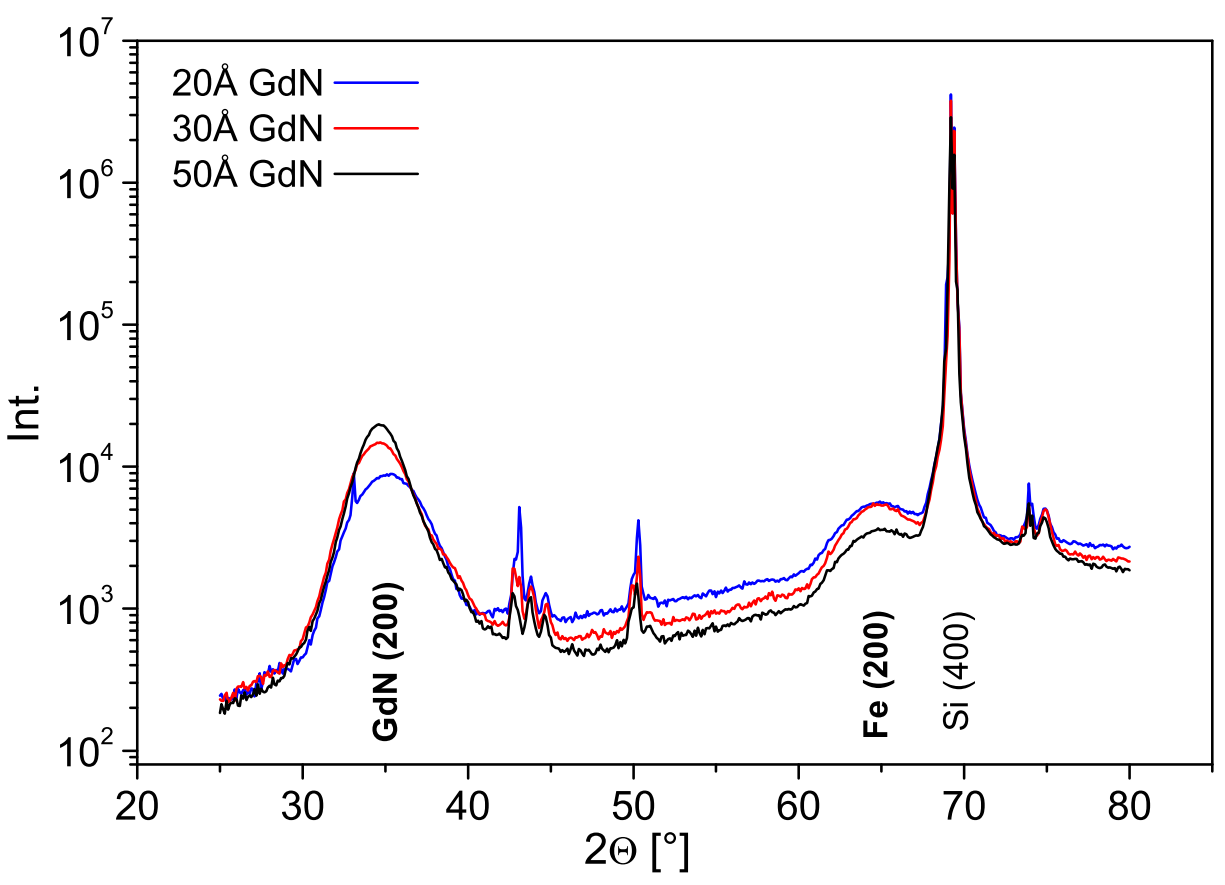

Figure 5.3: Bragg-Bretano X-Ray diffraction pattern $\left(\mathrm{CuK}_{\alpha}\right)$ of the $\mathrm{GdN} / \mathrm{Fe}$ multilayers prepared on $\mathrm{Si}(100)$ substrates. The three measurements belong to GdN/Fe multilayers with GdN thicknesses of $20 \AA, 30 \AA$ and $50 \AA$ and 88,72 and 52 bilayers, respectively. The Fe thickness was $25 \AA$ for all three samples. The small sharp peaks are caused by the sample holder

Bretano) the scattering vector is parallel to the surface normal and therefore the lattice constant is only measured along the scattering vectors direction providing no information about the spacing in the in-plane direction. A larger value of the interplanar distance in the out of plane direction could have its origin in a tetragonal distortion induced by the mismatch between the Fe and the GdN. Comparing the bulk lattice constants of Fe and GdN the closest match for the (100) planes can be achieved by rotating one plane of about $20^{\circ}$ around the surface normal. The mismatch in this configuration is still $8 \%$ and the larger spacing between the GdN planes along the surface normal indicates that this missmatch induced by the iron leads to a compressive stress in the GdN at the interface. To prove this tetragonal distortion a special sample was prepared onto a 500nm thin SiN membrane giving the possibility to apply the $\Theta-2 \Theta$ geometry in transmission as well. In this 


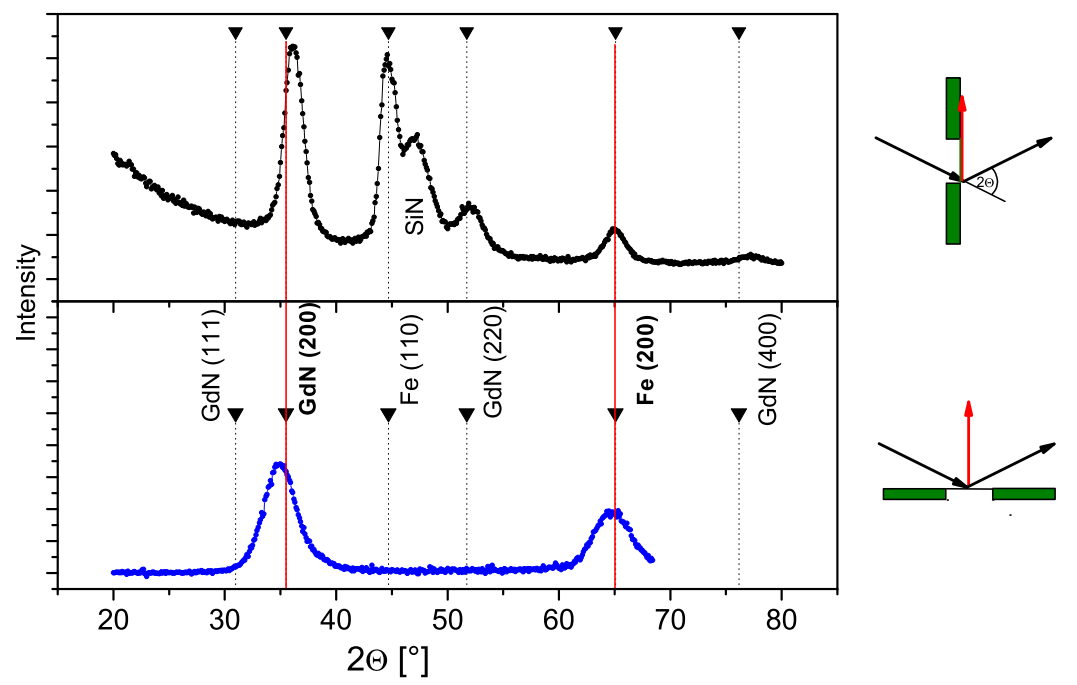

Figure 5.4: XRD pattern of a GdN/Fe multilayer on a SiN membrane (d = $500 \mathrm{~nm}$ ) with scattering vector out of plane (lower panel) and in plane (upper panel). The shift of the GdN (200) peak is clearly visible, whereas the Fe (200) peak is found to be always at the same value expected for bulk Fe.

case the scattering vector can be rotated into the sample plane by simply rotating the sample by $90^{\circ}$. The result is shown in Fig. 5.4. Calculating the lattice constant from the measurement in the "normal" Bragg-Bretano geometry with the scattering vector out of plane one obtains a lattice constant of $0,513 \mathrm{~nm}$ in good agreement with the other multilayers on Si (100) substrates. By rotating the sample by $90^{\circ}$ the scattering vector is now in plane and one observes a shift of the GdN (200) reflection to higher angles. The corresponding plane spacing is found to be $0,497 \mathrm{~nm}$ which is a direct evidence of the tetragonal distortion of about 3\%. For the Fe (200) peak no significant shift can be observed indicating that the Fe interface remains nearly undistorted. As an additional result the texture of the samples can be seen clearly. In the out of plane geometry only the GdN (200) and Fe (200) peaks are visible, whereas in the in plane geometry additionally the Fe (110) and the GdN (220) peaks are showing up. The GdN (111) peak is neither seen in nor out of plane. That means that these samples grow along the surface normal in (100) oriented columns that are rotated randomly with respect to each other in plane. The interface of the multilayers is found to be 


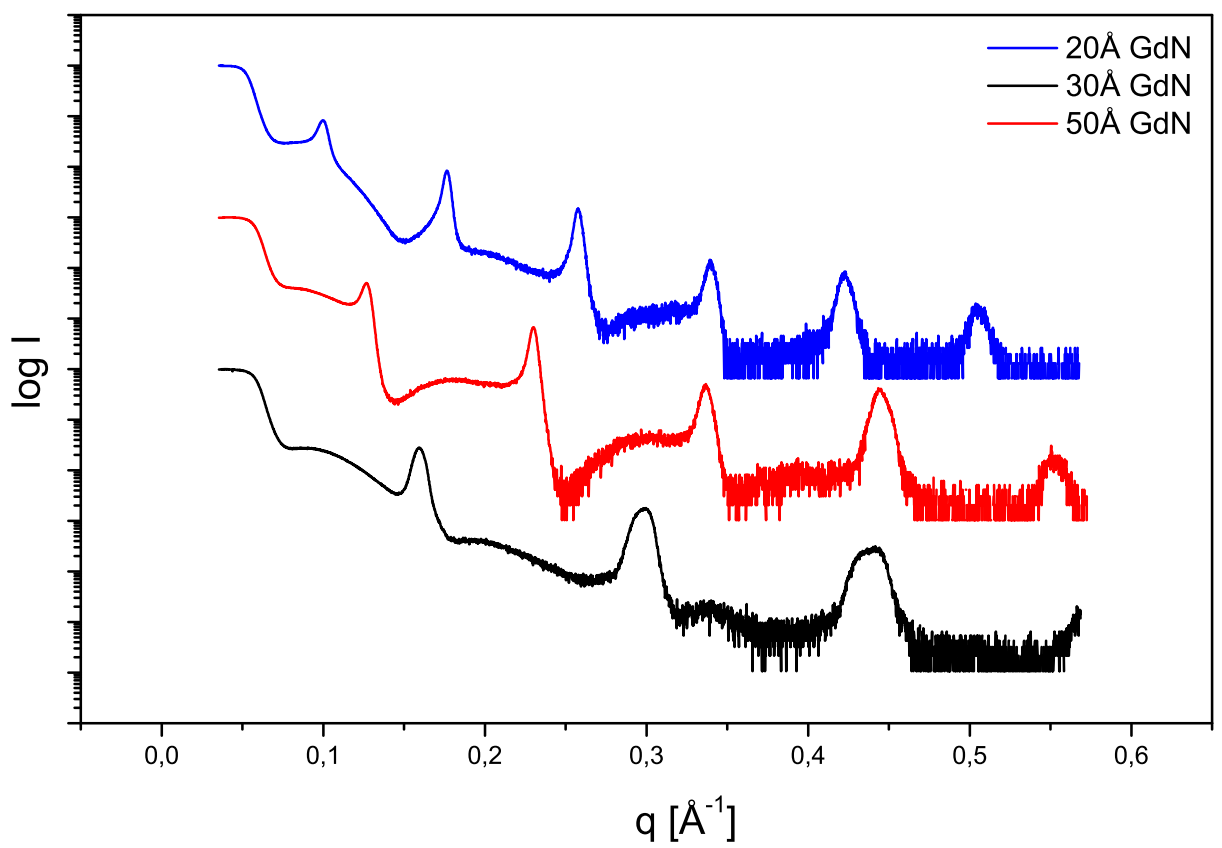

Figure 5.5: Small angle diffraction of the multilayers prepared on Si (100) substrates. The clear defined satellites testify the sharp interfaces of the multilayers.

well defined with a RMS roughness of about 1ML. In Fig. 5.5 the small angle diffraction patterns are shown for the three multilayers. All three diffraction patterns show clearly defined satellites as one expects for a multilayer with sharp interfaces. A more detailed analysis was done in [38].

\subsection{Macroscopic Magnetization}

As mentioned above the Fe/GdN multilayers always form an artificial ferrimagnet in their ground state and one expects a compensation point at a distinct temperature depending on the thickness ratio of the layers. Since the thickness of the GdN layers is very small one could expect finite size effects that reduce the Curie temperature as reported by Farle et al. [72]. They found a reduction of the Curie temperature of ultra thin Gd films of up to $50 \%$ when the thickness of the Gd was below 10 ML. A determination of the Curie temperature for GdN thin films with a thickness between $500 \AA$ and $20 \AA$ was made in [38]. In contrast to $\mathrm{Gd}$ the reduction of $T_{C}$ was 


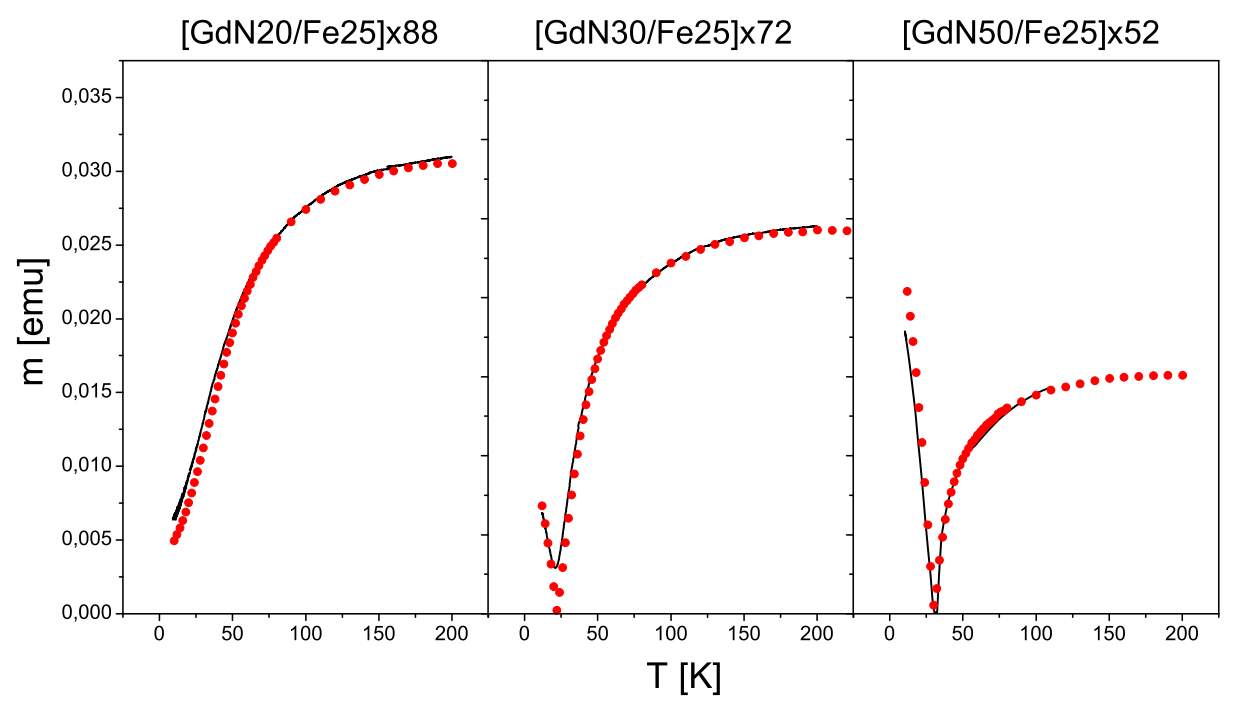

Figure 5.6: Magnetic moment vs. temperature of the multilayers in an external field of 200Oe. The red dots represent the samples on kapton foils, the black lines belong to the reference samples on $\mathrm{Si}(100)$.

only 3 Kelvin for a $20 \AA$ film and finite size effects are not considered here. Taking the density of the materials into account the ratio of the number of atoms in a GdN20 $\AA / F e 25 \AA$ bilayer would be 3:1 and therefore the magnetic moments at low temperatures are expected to be $6,6 \mu_{B}$ for the Fe layer and $7 \mu_{B}$ for the GdN normalized to the number of Gd atoms. Accordingly, the sample [GdN20/Fe25]x88 should have the lowest compensation point whereas this point must be shifted upwards in temperature for the other two samples $[\mathrm{GdN} 30 / \mathrm{Fe} 25] \times 72$ and $[\mathrm{GdN} 50 / \mathrm{Fe} 25] \times 52$ that have a ratio of $7 \mu_{B}: 4.4 \mu_{B}$ and $7 \mu_{B}: 2.75 \mu_{B}$, respectively. The macroscopic magnetization vs. temperature was measured with a Vibratin Sample Magnetometer (VSM) in a field of 200Oe to ensure that all samples have the same magnetic orientation. Figure 5.6 shows the results for the three samples. As expected from the thickness ratio the sample with a $50 \AA$ GdN layer has the highest compensation point which is located at $31 \mathrm{~K}$. For the sample with $30 \AA$ layer thickness the compensation point is considerably lower and for the $20 \AA$ sample it is too low to be reached within the temperature range of the VSM. For temperatures higher than $T_{C, G d N}$ one would expect the contribution of the Fe magnetic 
moment reduced by the contribution of the antiparallel polarized GdN at the interface due to the Fe. The total moment is therefore smaller even at $200 \mathrm{~K}$ than the moment expected from bulk Fe. Also the decrease of the total moment towards lower temperatures is more pronounced than one would expect from the temperature dependence of the magnetic moment of bulk GdN. Since all measurements concerning the structure had to be done with samples prepared on Si substrates while the XMCD measurements are carried out with samples prepared on Kapton, the macroscopic magnetization of both types was measured to ensure that the magnetic properties are the same. In Fig. 5.6 the black line corresponds to the samples on Si and the red dots to those on Kapton. The match between the samples is very good and shows that the multilayers can be grown in a good quality independently of the substrate. Assuming that the magnetic moment of the iron layers does not change in this temperature range the contribution of the GdN layers is the difference between the constant part of iron and the total measured moment. This simple picture does only hold if the layers are in a single domain state and there are no magnetically dead layers at the interface or any other structural or chemical deviations that affect the magnetic moment of one or both materials.

The necessary separation of the magnetic contributions can be done by XMCD measurements either at the Gd-edges, the Fe-edges or both. As mentioned above, the temperature dependence of the iron layer magnetization is expected to be very small and the more interesting part is the contribution of the GdN. Although the M-edges of the Gadolinium seem to be a good choice for such a XMCD experiment since the magnetization of GdN is dominated by the $4 \mathrm{f}$ moment the measurement was done at the L-edges for two reasons. The L-edges are located in the high energy range $(h \nu>7 \mathrm{keV})$ and the transmission in this range is much higher, which gives the possibility to prepare samples with up to 88 bilayers. By increasing the number of bilayers, interface effects like the expected magnetic polarization of the GdN by the iron are accessible with better signal to noise ratio. The $5 \mathrm{~d}$ moment probed by the L-edge XMCD has, due to the intra-atomic coupling, the same temperature dependence as the $4 \mathrm{f}$ moment. In order to extract the pure temperature dependence of the GdN magnetization the measurements were done in zero 
field to avoid an impact by the external field. The measurements of the Gd $\mathrm{L}_{2}$-edge XMCD were done at the beamline ID24 of the ESRF (Grenoble). In consideration of the fact that the magnetic dichroism in transmission is only sensitive to the magnetic moment projected onto the direction of the x-ray beam, the samples' magnetic orientation must be aligned into a defined direction before the measurement starts. This was done by applying a magnetic field of $4 \mathrm{kOe}$ generated by an electromagnet parallel to the x-ray beam axis at low temperature. For the samples with $50 \AA$ and $30 \AA \operatorname{GdN}$ thickness this procedure leads to a parallel aligned GdN net moment, since the temperature is below the compensation point. In case of the $20 \AA$ sample the compensation point could not be reached, because it is below $5 \mathrm{~K}$. Therefore the $\mathrm{L}_{2}$-edge $\mathrm{XMCD}$ has the opposite sign in the measurement, but was reversed subsequently. The field was set to zero and the magnetic dichroism was measured from low to high temperature for the samples with GdN thicknesses of $50 \AA, 30 \AA$ and $20 \AA$. Since the measurements were done in zero field and only the temperature dependence is of interest the norm of the measured $\mathrm{XMCD}$ is plotted in Fig. 5.7 to clarify the results. Because the magnetic dichroism signal is normalized to the isotropic absorption $\mu=\left(\mu^{+}+\mu^{-}\right) / 2$ at the $\mathrm{L}_{2}$ edge the dichroism is a measure of the magnetic moment of the $5 \mathrm{~d}$ spin moment per atom. From this point of view the first eye-catching result is that the moment is different in magnitude for all three samples at every temperature. At high temperature the explanation of the thickness dependence is obvious and arises from the fact that even at room temperature the GdN has a magnetic moment at the interface due to the polarization by the iron. This polarization is only of short range and decreases fast with the GdN layer thickness. Since the isotropic absorption $\mu$ at the $\mathrm{L}_{2}$ does not depend on the magnetization but only on the amount of material that is probed by the beam, the thickness dependence of the normalized signal reflects that the layer is only magnetized at the interface and the core layers have almost no net moment. Consequently for a thicker layer only the $\mathrm{L}_{2}$ absorption is increased but the dichroism remains the same and the normalization to the $\mathrm{L}_{2}$ absorption results in a smaller signal. At room temperature the iron is the only source for polarizing the GdN and from the thickness dependence of the signal one can extract the polarization length assuming an exponential decay. 


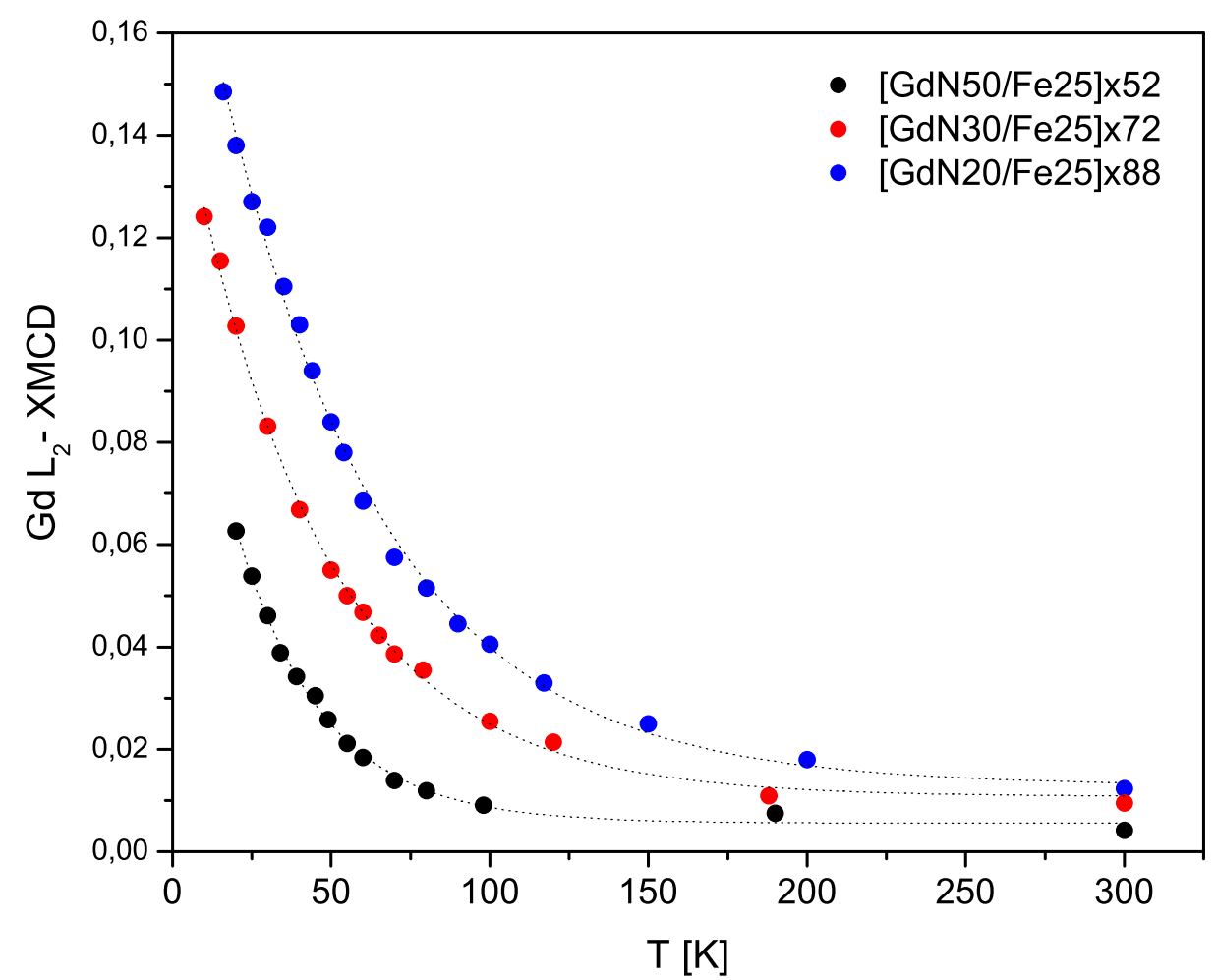

Figure 5.7: Normalized XMCD in zero magnetic field at the $\mathrm{Gd}_{2}$-edge for different GdN thicknesses. The dotted line are fits using an exponential decay

Here we find an interfacial polarization length of about $5 \AA$ in GdN which is equal to the unit cell in GdN. The signal strength at room temperature is about $10 \%$ of the value found at low temperatures.

At low temperature $\left(T<T_{C, G d N}\right)$, when the GdN is fully magnetized, one would not expect a thickness dependence of the XMCD, since all GdN layers should have nearly the same moment. This is obviously not the case here. For thinner GdN layers the XMCD curves are shifted upwards in temperature and are of higher magnitude for low temperatures. This cannot be an effect of polarization due to the iron, since the polarization is superposed by the intrinsic spontaneous magnetic moment of $\mathrm{GdN}$ at low temperatures which is much greater. Even at $100 \mathrm{~K}$, which is well above the bulk Curie temperature $T_{C, G d N}$, the GdN layers in all three samples have a distinct magnetization that is higher than the iron induced contribution at room temperature.

An enhancement of the ordering temperature of a thin Ni Film in mag- 
netically coupled $\mathrm{Co} / \mathrm{Cu} / \mathrm{Ni}$ samples was reported by Srivastava et al. [73], a theoretical description was given by Jensen et al. [74]. The increased magnetization above $T_{N i}^{\star}$ of the uncoupled film is explained with the fact that the magnetic coupling between $\mathrm{Co}$ and $\mathrm{Ni}$ by the indirect exchange interaction across the $\mathrm{Cu}$ film suppresses spin fluctuations in the $\mathrm{Ni}$ film. For an uncoupled free $\mathrm{Ni}$ thin film of the same thickness the fluctuations diminish the magnetization significantly and the Curie temperature of the free film is already below the bulk Curie temperature of Ni. In contrast to the shift observed here, their observed enhanced ordering temperature $T_{N i}^{\star}$ is still below the Curie temperature of bulk Ni. Wang and Mills have discussed a similar problem as an onset of long-range order in a superlattice model in the frame of the Landau-Ginzburg theory [75]. The model they consider is a superlattice of two films $\mathrm{A}$ and $\mathrm{B}$ having bulk transition temperatures $T_{A}^{(\infty)}$ and $T_{B}^{(\infty)}$ where $T_{A}^{(\infty)}>T_{B}^{(\infty)}$. In the limit of film thicknesses for $\mathrm{A}$ and $\mathrm{B}$ of one monolayer the system behaves like a new material with a coherent magnetic structure having only one transition temperature that is located between $T_{A}^{(\infty)}$ and $T_{B}^{(\infty)}$. On the other hand for large thicknesses of $\mathrm{A}$ and $\mathrm{B}$ it is obvious that the two transition temperatures reappear and the system behaves like a superposition of the two bulk materials. In [75] several different scenarios of film thicknesses are discussed. For film thicknesses and ratio comparable to the thicknesses here they find a single magnetic phase transition with a slightly reduced transition temperature for A (here the iron film) and an enhanced magnetization of $\mathrm{B}$ (here GdN) above $T_{B}^{(\infty)}$. For the temperature dependence of the magnetization of film $\mathrm{A}$ the general trend of the curve is like the expected response of a ferromagnetic material. Film B does not have a clearly definable transition temperature anymore. Its magnetization is broadened over a large temperature range. This is comparable to the $\mathrm{L}_{2}$-edge results in Fig. 5.7. Although the observed increase of magnetization above the Curie temperature of bulk GdN for smaller thicknesses may be ascribed to an increased $T_{C, G d N}$ in the multilayers, in the background of Wang's results it is not necessarily reasonable to specify a $T_{C}$ for the GdN layers. Keeping in mind that the magnetic properties of GdN are also sensitive to the next neighbor distance and the fact that the iron induces a lateral compressive stress at the interface the smaller in-plane Gd spacing might ad- 


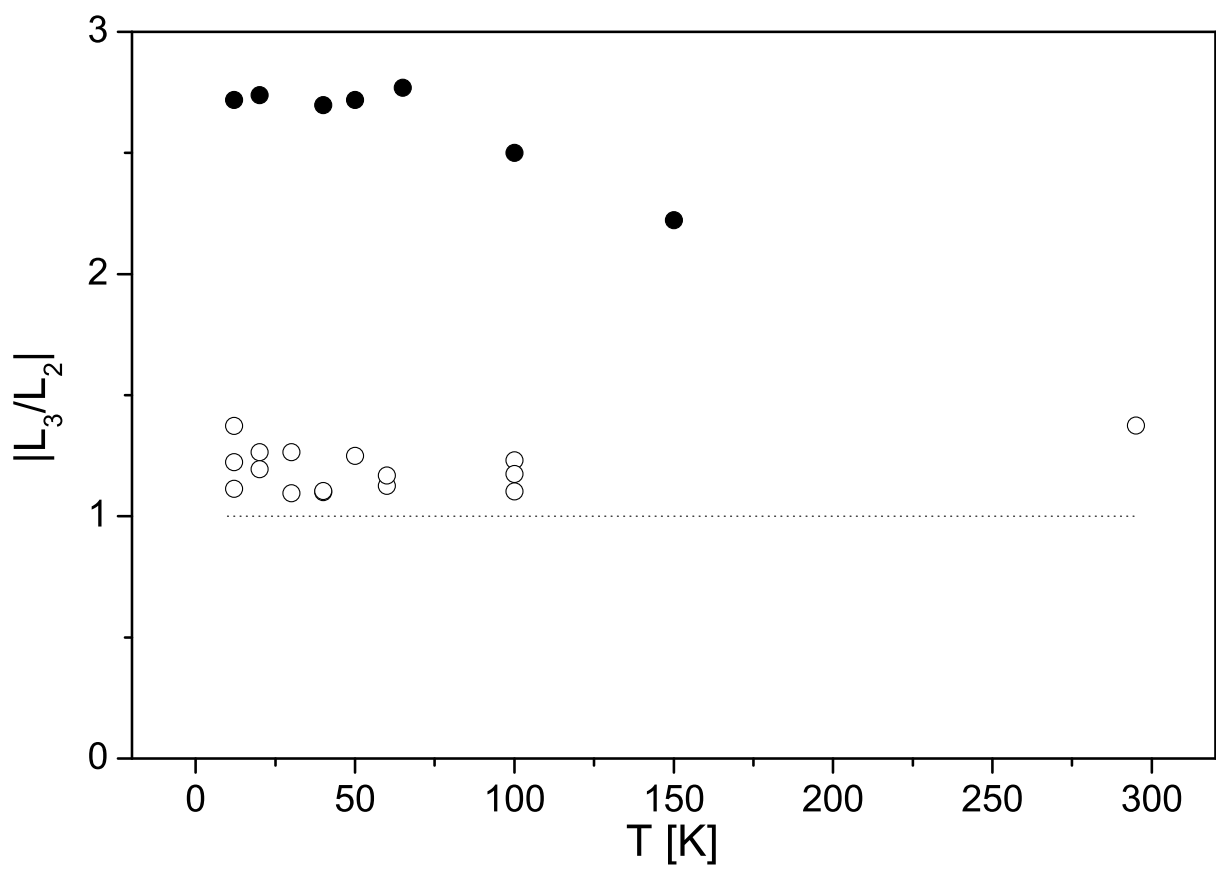

Figure 5.8: The $\left|L_{3} / L_{2}\right| \mathrm{XMCD}$ ratio for the multilayers (open circles) is different from the ratio observed in the single GdN film (full circles). For all multilayers systems a value of close to 1 is observed, which is expected for ferromagnetic Gd.

ditionally affects the magnetization of the GdN layers. The smaller in plane lattice constant could lead to an increased $J_{1}$ in Methfessel's picture [33]. In chapter 4.5 the smaller amplitude of the $\mathrm{L}_{2} \mathrm{XMCD}$ was attributed to a decreased $J_{1}$ and one could expect a increased $\mathrm{L}_{2}$ in the multilayers. In Fig. 5.8 the $\left|L_{3} / L_{2}\right|$ XMCD ratio for the $2000 \AA$ GdN film on Kapton from chapter 4.5 is shown (full circles). The open circles reflect the $\left|L_{3} / L_{2}\right|$ ratio of the multilayers. Independent of the GdN thickness and temperature all ratios are close to 1 as usually found for Gd or ferromagnetic Gd alloys. This result suggest that the enhanced magnetization above $T_{C, G d N}$ could also be a structural effect caused by the compressive stress at the interface. At this point further experiments are needed. The $\left|L_{3} / L_{2}\right|$ ratio of a single GdN film grown at high temperature with a lattice constant close to the bulk value should be almost the same as observed in the multilayers. The substitution of iron with a structural similar but nonmagnetic material like $\mathrm{Cr}$ in the multilayers would help to separate the polarization of the GdN by the iron 
from the structural part.

The general trend of the variation of the XMCD signal with temperature can be described by an exponential decay and is represented by the dotted lines in Fig. 5.7. Underlying these results and the fact that the iron magnetization changes only little with temperature, one can model a magnetic profile of the samples in the following way. At room temperature the Fe layers have the bulk magnetization value of $1707 \mathrm{emu} / \mathrm{cm}^{3}$ [61] and the $\mathrm{GdN}$ is magnetically polarized by the iron layers with a decay of $5 \AA$. With decreasing temperature the GdN magnetization follows the temperature dependence of the exponential function determined from the $L_{2}$-edge.

$$
M(T)=M_{S} \cdot e^{-\frac{T}{\tau}}+M_{P}
$$

Here $M_{S}$ is the saturation magnetization of GdN and is set to the bulk value of $2020 \mathrm{emu} / \mathrm{cm}^{3}$. The parameter $\tau$ is the decay length of the exponential fit and can be seen as an indicator of an enhanced exchange in the GdN layer. The value of $\tau$ increases with decreasing GdN layer thickness from 27K to 51K (Table 5.1). Since the microscopic mechanism that connects the temperature dependence of the XMCD to Eq. (5.1) is not known, $\tau$ has to be seen as a phenomenological value, but reflects the increase of $T_{B}$ in the model of Wang and Mills for decreasing film thicknesses. $M_{P}$ is set to the effective interfacial polarization induced by the iron at room temperature that was determined from the $\mathrm{L}_{2}$-edge XMCD in Fig. 5.7. Using $M(T)$ from equation (5.1) for GdN and assuming a constant magnetization across the $\mathrm{Fe}$ layers we obtain the magnetic profile of the multilayers. The result for the GdN50/Fe25 sample at $\mathrm{T}=10 \mathrm{~K}$ and $\mathrm{T}=90 \mathrm{~K}$ is shown in Fig. 5.9. The total magnetic moment of the samples was calculated using this model with the layer thicknesses determined from x-ray small angle diffraction and a sample area of $1 \mathrm{~cm}^{2}$ reflecting the real dimensions of the measured samples. In Fig. 5.10 the norm 1 of the calculated moment represented by the red curves is compared with the measurements for the three samples. The calculation

\footnotetext{
${ }^{1}$ Due to the fact that the temperature dependence of the magnetization was carried out in a small field of $H=2000$ e the samples magnetization rotates by $180^{\circ}$ when the temperature crosses the compensation point and therefore one obtains only a positive total moment.
} 


\begin{tabular}{|c|c|c|}
\hline Sample & $\tau$ & $M_{P}$ \\
\hline$[\mathrm{GdN} 20 / \mathrm{Fe} 25] \times 88$ & $27 \mathrm{~K}$ & $5 \%$ \\
{$[\mathrm{GdN} 30 / \mathrm{Fe} 25] \times 72$} & $43 \mathrm{~K}$ & $7 \%$ \\
{$[\mathrm{GdN} 50 / \mathrm{Fe} 25] \times 52$} & $51 \mathrm{~K}$ & $7 \%$ \\
\hline
\end{tabular}

Table 5.1: Results for the fit of the temperature dependence of the $\mathrm{L}_{2}$-edge XMCD with an exponential decay for all three samples. The fit is normalized to $M_{S}=$ $2020 \mathrm{emu} / \mathrm{cm}^{3}$ a $T=0 K . M_{P}$ is the percentage interface polarization of the GdN at room temperature

does not only render the general trend of the magnetic moment, but also the magnitude of the magnetization and the compensation points fit nearly perfectly. The perfect match of the absolute magnetic moment of the samples with the model states that the magnetic moment of the bilayers from the model is correct. If there would be a magnetic dead layer at the interface or a disturbed magnetic moment due to chemical mixing the absolute measured

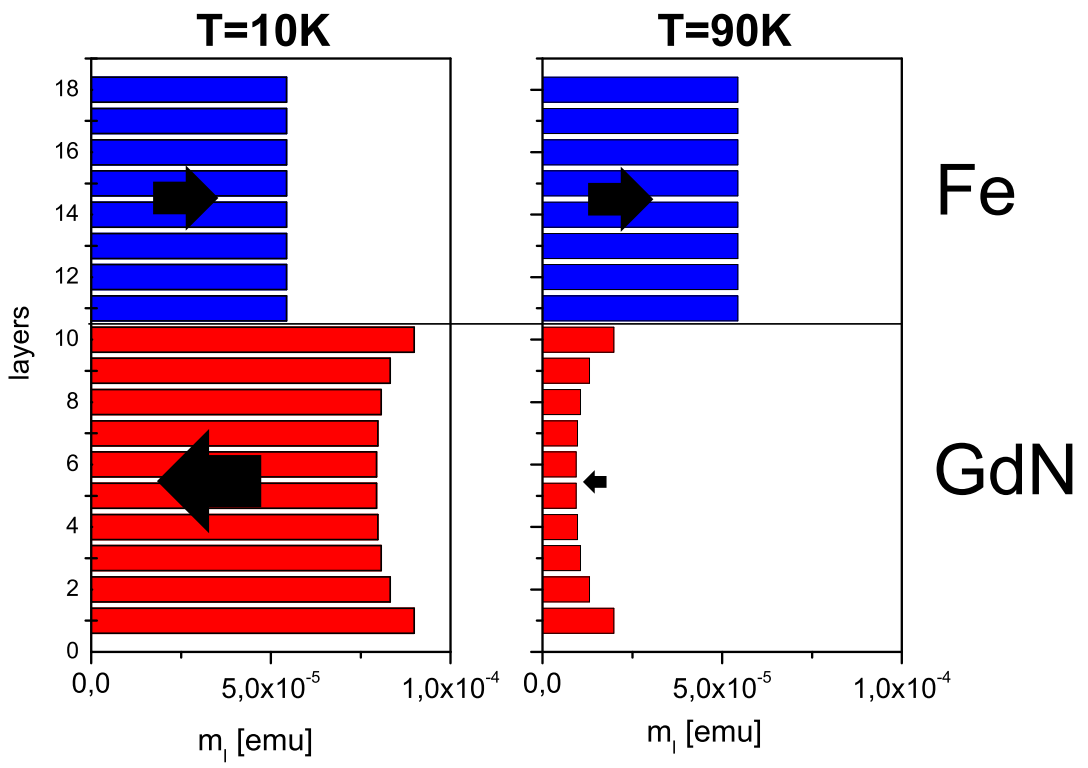

Figure 5.9: Model of the magnetic profile of a GdN50/Fe25 multilayer for temperatures below $(T=10 K)$ and above $(T=90 K) T_{C, G d N}$. The bars represent the layers magnetization of the corresponding material. The Fe and the GdN moments are coupled antiparallel. 


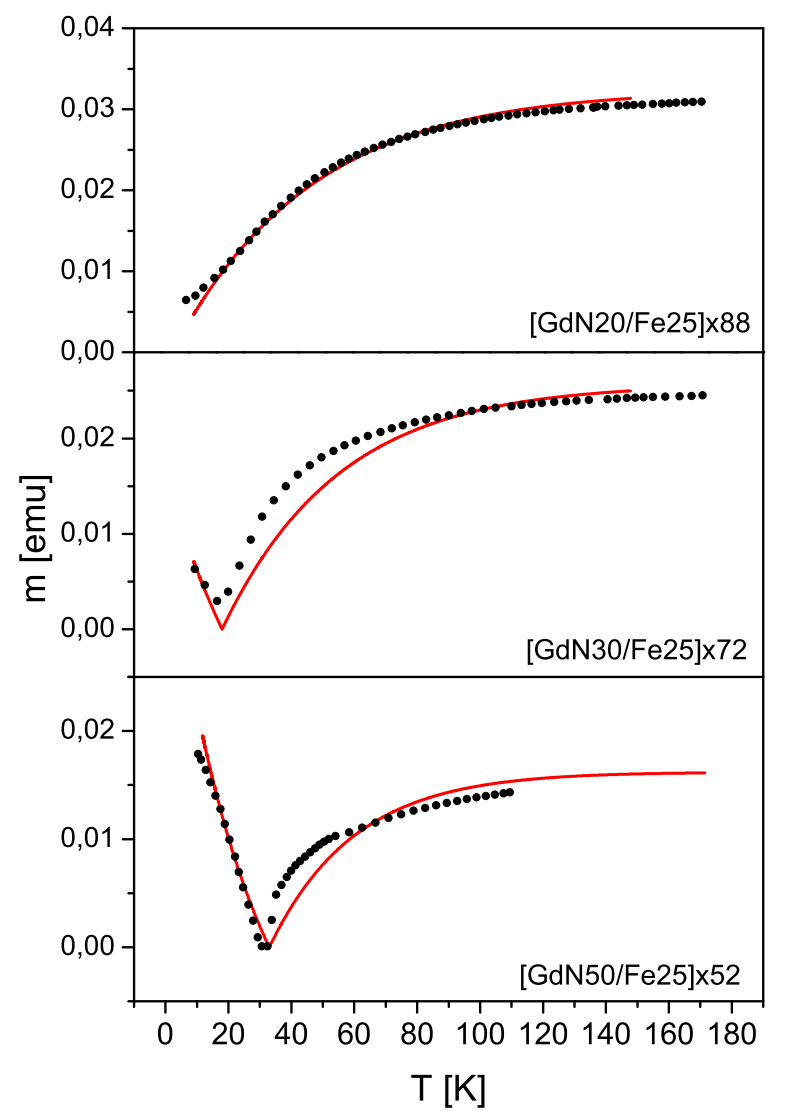

Figure 5.10: Comparison between the absolute measured magnetic moment of the GdN/Fe multilayers on $\mathrm{Si}(100)$ (see figure 5.6) and the calculated moment (dots) based on the model described in the text (straight lines). Besides small differences the model reproduces the $\mathrm{M}(\mathrm{T})$ curves very well.

moment should differ from the calculated. This is obviously not the case here and can be seen as an advise for the sample quality.

\section{$5.4 \quad$ Field dependence}

As mentioned in section 5.1 Motokawa's calculation [63] predicts a critical magnetic field $H_{C}$ that is needed to start a twist in a antiferromagnetically coupled superlattice. The calculated thickness dependence of the critical field was compared with experimental results obtained for a $70 \AA \mathrm{Fe} / 200 \AA \mathrm{GdFe} / 70 \AA$ Fe trilayer [62]. With decreasing GdFe thickness the critical field starts to 


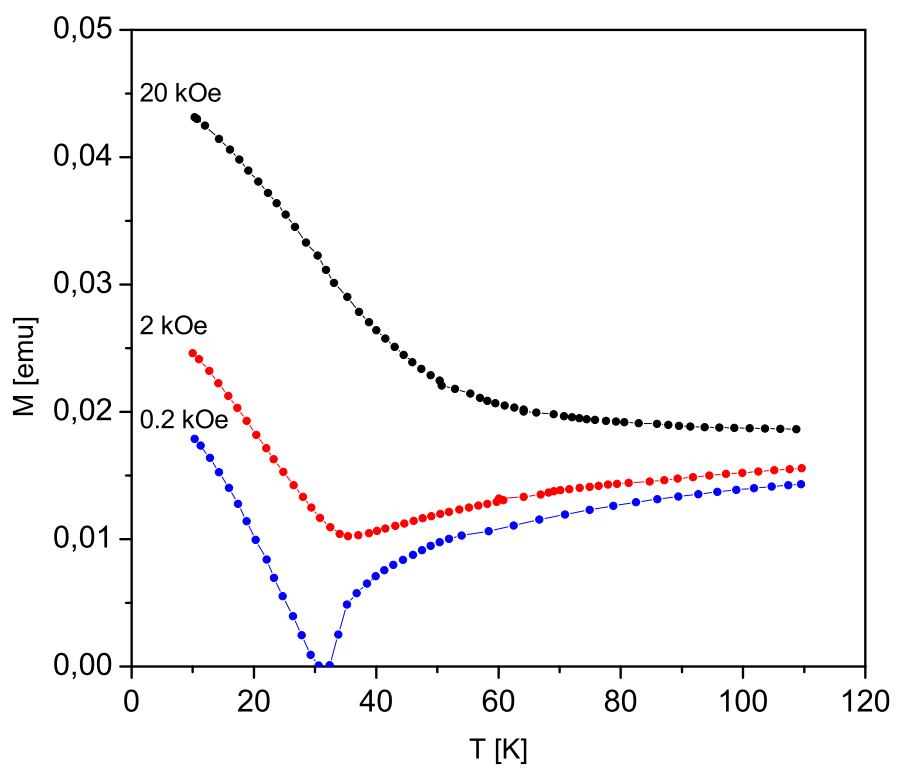

Figure 5.11: Field dependent $\mathrm{M}(\mathrm{T})$ curves for the [GdN50/Fe25]x25 multilayer in an external field of $0.2,2$ and $20 \mathrm{kOe}$. The compensation point is suppressed completely in a field of $20 \mathrm{kOe}$.

increase and diverges at about $100 \AA$. In the present system the layer thickness is much smaller than in the system examined by Motokawa et al. and one would expect a simple collinear coupling that should not be affected by an external field. However, the results for the GdN/Fe samples are different, as it will be discussed in the following part. In Fig. 5.11 the temperature dependence of the magnetization of the [GdN50/Fe25]x52 sample is shown for different external fields. The compensation point is completely suppressed by an external field of $20 \mathrm{kOe}$ and even in $2 \mathrm{kOe}$ the net magnetization does not vanish anywhere. In Camley's calculation for a Gd/Fe system the Gd aligned phase persists at low temperatures until the external field exceeds $\sim 10 \mathrm{kOe}$ even for weak coupling. In a aligned phase the magnetic moment should not depend on the external field, like in the model of Motokawa were a external field lower than a critical value $H_{C}$ does not induce a twist in the layers magnetization. In Fig. 5.11 even a external field smaller than $10 \mathrm{kOe}$ always increases the magnetic moment significantly regardless of the temperature and therefore the value of $H_{C}$ in the GdN/Fe multilayers must 
have a distinct smaller value compared to Camley and Motokawa. This may be a consequence of the smaller $T_{C, G d N}=59 \mathrm{~K}$ compared to $T_{C, G d}=292 \mathrm{~K}$ in a $\mathrm{Gd} / \mathrm{Fe}$ system.

The measured field dependence in Fig. 5.11 can belong to a variety of different angular distributions having all the same projection onto the field axis. Although XMCD measurements are not generally suited to gain information about an angular distribution, the separation of the Fe and GdN layer contributions to the multilayer magnetization can shed light on magnetic state of the samples. In chapter 3 it was noted that the magnetic circular dichroism is a measure of the projection of the spin (orbital) moment onto the direction of the x-ray beam. A change in the signal strength is connected either to a change in the magnitude of the magnetic moment or to change of the angle between its direction and the beam or both. For low magnetic fields one can assume a collinear alignment of the magnetization to the field axis and the temperature dependence is only connected to the magnitude of the magnetic moment. Applying higher fields causes a twist of the relative magnetization orientation of the layers giving a different projection of the net moment onto the axis of the beam. Although a direct measurement of the angular distribution is not possible with the XMCD experiments made here, differences in the temperature dependence of the magnetization of the Fe and GdN layers gives additional information about the relative angular alignment between them.

\subsubsection{Dichroism of the Gd L-edges}

The magnetic dichroism of the $\mathrm{Gd} \mathrm{L}_{2}$ - and $\mathrm{L}_{3}$-edges was measured for different GdN layer thicknesses at the dispersive beamline D11 at LURE. Since the Fe K-edge absorption is located at $7110 \mathrm{eV}$ and the $\mathrm{Gd} \mathrm{L}_{3}$-edge absorption at $7242 \mathrm{eV}$ the energy range was chosen in a way that both, the Fe $\mathrm{K}$ - and the $\mathrm{Gd} \mathrm{L}_{3}$-edge could be measured at the same time. This has the advantage that both XMCD signals are recorded simultaneously and the Fe K-edge XMCD can be used to reference the relative signal strength of the Gd L-edge XMCD, since the Fe K-edge XMCD probes the magnetic polarization of the Fe 4p states, which is proportional to the $3 \mathrm{~d}$ polarization of the Fe. Although a 


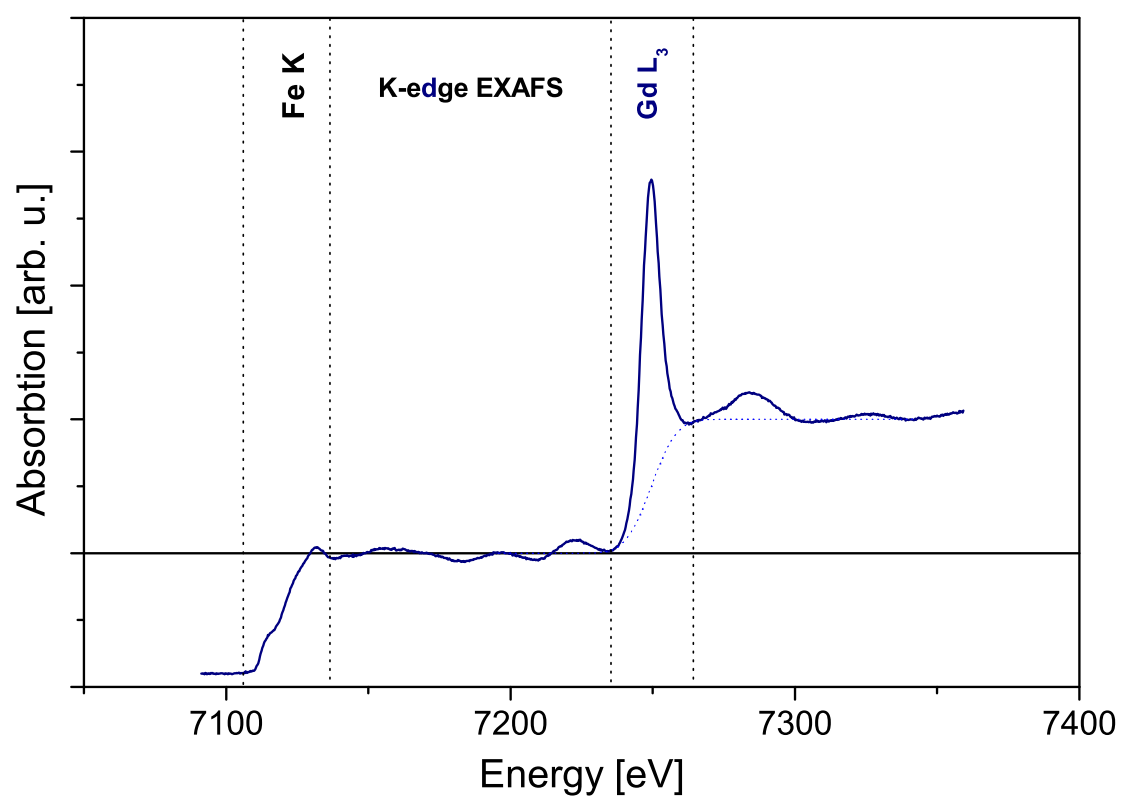

Figure 5.12: Isotropic x-ray absorption measured in transmission of the Fe Kedge and $\mathrm{Gd} \mathrm{L}_{3}$-edge of the [GdN50/Fe25]x52 sample on kapton. The oscillations between the $\mathrm{Fe} \mathrm{K}$-edge and the $\mathrm{Gd} \mathrm{L}_{3}$-edge are EXAFS oscillations from the $\mathrm{Fe}$. The dotted curve is the step function used to normalize the XMCD

direct comparison of the magnetic moment is not possible due to the failure of the sum rules for the Gd L-edges (see chapter 3) a difference of the temperature dependence of both signals can give information about the relative angular alignment between them. In Fig. 5.12 the isotropic absorption of the [GdN50/Fe25]x52 sample is shown in a energy range of 7091-7359eV. The Fe $\mathrm{K}$-edge and the $\mathrm{Gd} \mathrm{L}_{3}$-edge are marked in-between the dotted vertical lines. The normalization of the XMCD signal to the volume of the probed material is conventionally done by scaling the absorption spectra to an edge jump of 1 (blue dotted line).

At room temperature only the Fe layers are ferromagnetic and the GdN is paramagnetic with a small ferromagnetic polarization at the interface that is antiferromagnetically coupled to the iron. In this case one observes a XMCD signal at the Fe K-edge that is known to belong to a parallel alignment of the Fe magnetization with the external field [14]. The signal from the $\mathrm{Gd} \mathrm{L}_{3}$ is very small and opposite in sign (Fig. 5.13). This reflects the AFM coupled polarization at the interface. Decreasing the temperature leads to a slight 


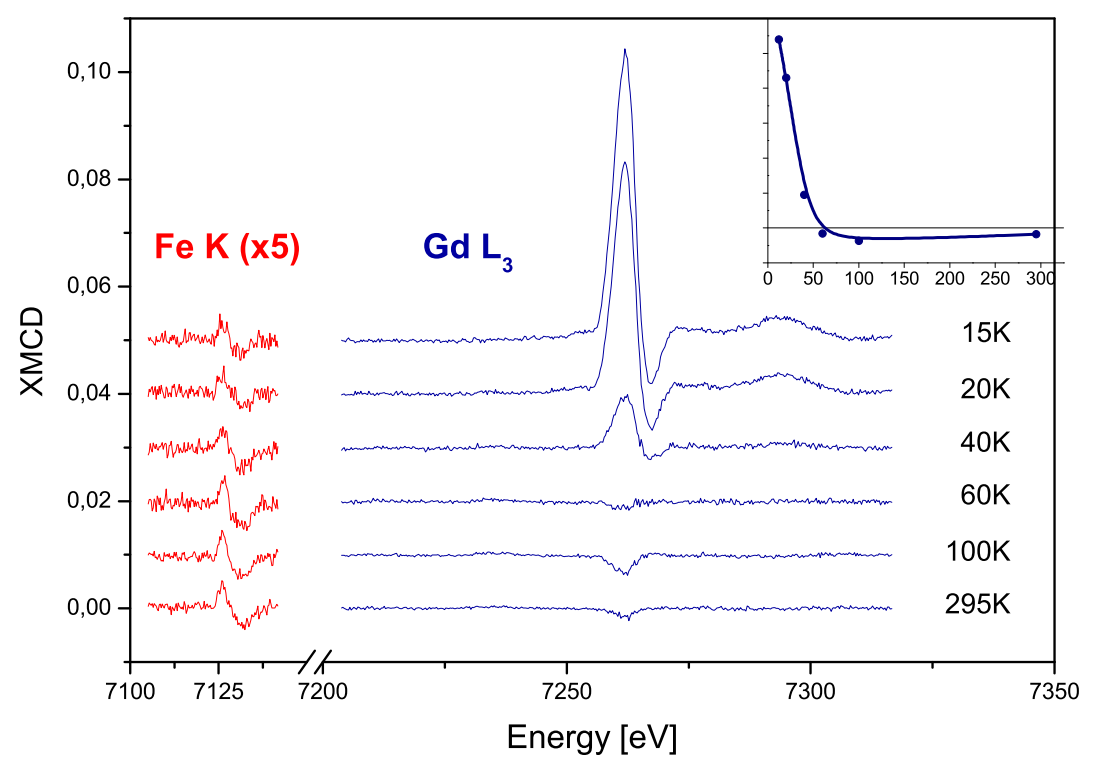

Figure 5.13: Temperature dependence of the normalize XMCD at the $\mathrm{GdN} \mathrm{L}_{3^{-}}$ edge and the XMCD from the Fe K-edge in an external field of $\pm 20 \mathrm{kOe}$ of a [GdN50/Fe25]x52 multilayer. The Gd $\mathrm{L}_{3} \mathrm{XMCD}$ amplitude is plotted against the temperature in the inset

increase of the $\mathrm{Gd}$ signal down to the $\mathrm{T}_{C}$ of the $\mathrm{GdN}$. When the temperature drops below $\mathrm{T}_{C}$ the $\mathrm{Gd}$ signal changes its sign and then increases very fast. This is the point where the GdN layer magnetization starts to rotate into the applied field and and the net magnetization of the GdN is aligned parallel to the field. Remembering the Gd/Fe system and Camley's model one would expect at the same time a change in the Fe signal since the system changes from the Fe aligned into the Gd aligned or twisted state. However, the K-edge signals in Fig. 5.13 only show a small decrease when the temperature drops below $T_{C, G d N}$. It can be concluded that only the GdN magnetization rotates into the field and the Fe magnetization stays nearly parallel to the field; one observes neither the GdN aligned state (where the Fe magnetization should be oriented antiparallel to the field) nor the twisted state (the Fe signal should vary in strength). This result confirms the assumption that the coupling in GdN/Fe must be distinctly smaller than in the Gd/Fe system that was made from the macroscopic measurements.

A smaller GdN layer thickness should lead to a change in the tempera- 


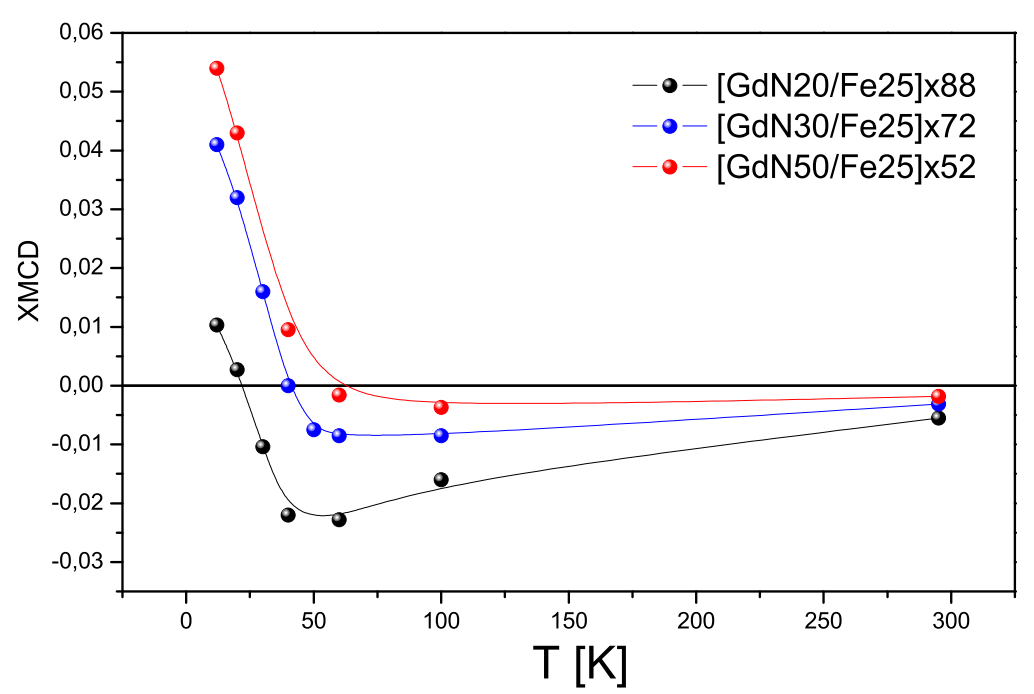

Figure 5.14: Temperature dependence of the Gd $\mathrm{L}_{3}$-edge XMCD amplitude for different GdN layer thicknesses in an external field of $20 \mathrm{kOe}$

ture dependence of the $\mathrm{Gd} \mathrm{L}_{3} \mathrm{XMCD}$, since Motokawa's model predicts an increasing critical field $H_{C}$. The first obvious difference in Fig. 5.14 between the $50 \AA$ and the $20 \AA$ sample is that in the paramagnetic range of the GdN the increase of the (negative) XMCD signal with decreasing temperature is much larger. This in agreement with the results from the remanently measured XMCD at the $\mathrm{L}_{2}$-edge. In contrast to the remanent measurements the $\mu^{+}$and $\mu^{-}$absorption spectra were achieved by flipping the magnetic field $( \pm 20 \mathrm{kOe})$ parallel to the direction of the beam. In the temperature range well above $T_{C, G d N}$ the evolution of the XMCD signal is comparable to the zero-field situation, because the polarized magnetization in the GdN is small and thus the Zeeman energy in the external field is not large enough to cause a significant rotation of the GdN layer magnetizations. With increasing magnetization the Zeeman term gets a larger influence and the GdN layer magnetization tries to minimize the Zeeman energy by partly rotating into the field but is choked by the exchange coupling in the GdN itself and the exchange at the interface. In the region below $T_{C, G d N}$ the XMCD changes in sign, which is related to a reversal of the projected net moment of the GdN layer. I should be reminded that for all three samples the Fe K-edge signal 


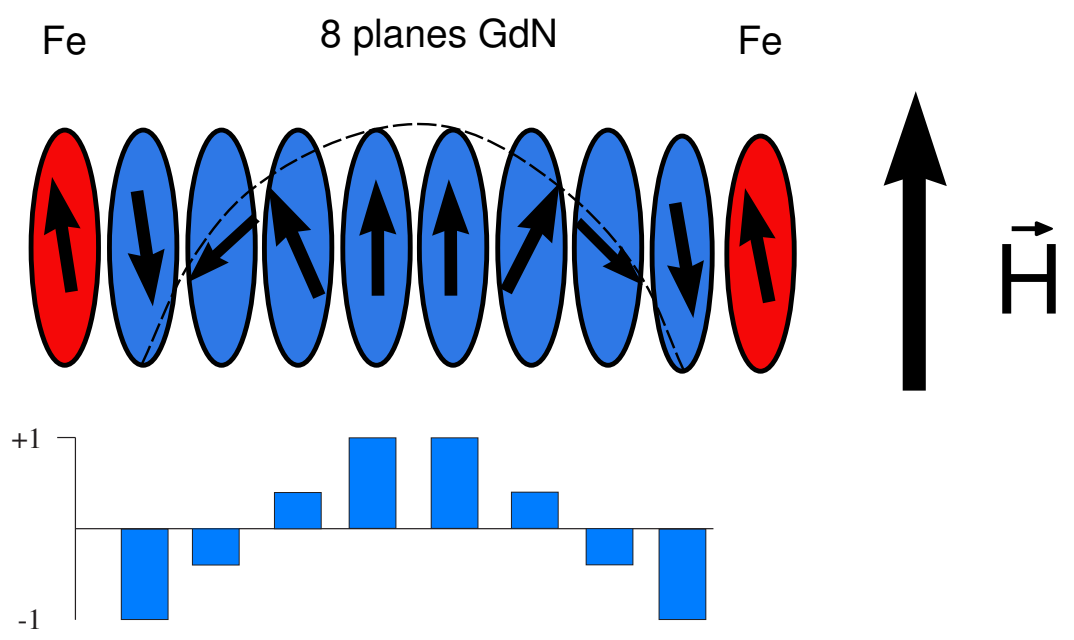

Figure 5.15: Model of the angular distribution of the magnetization in the [GdN20/Fe25] multilayer in an applied field of 20kOe. Only one monolayer of iron is drawn at the interfaces since all iron layers assumed to have the same angle. The dotted line is a guide to the eye. The bars represent the magnitude of the projected moment

remains nearly unchanged besides a slight decrease . Another obvious difference to the remanent measurements is that the magnitude of the XMCD at low temperatures is reversed for the samples. In Fig. 5.7 the sample with a $20 \AA$ GdN layer has the largest signal at $10 \mathrm{~K}$ whereas the signal of the same sample is almost zero here. This is an effect induced by the external magnetic field and can be explained by the field-induced angular distribution of the magnetization in the GdN layer. At the interface to the iron layer the exchange coupling is supposed to be larger than in the interior of the GdN due to the hybridization with the iron and the angle between the magnetization of the iron and the interfacial atomic plane of the GdN should be in the order of $180^{\circ}$. Inside the GdN the exchange coupling is much smaller and the magnetization in the atomic planes can rotate more easily into the field. $20 \AA$ GdN consists of nominally 8 atomic planes. A configuration with a zero projection can be constructed by to ferromagnetically aligned inner planes and two antiferromagnetically aligned interfacial planes. The remaining 4 planes rotate between the interfacial and the inner planes with almost no net projection. A greater GdN layer thickness leads to more ferromagnetically aligned inner planes, since the angular change in the GdN layers starting 
from the interface should be nearly the same for all layer thicknesses or even greater with increasing GdN thickness. Thus the XMCD increases with increasing GdN layer thickness. This model is of course not without ambiguity, because there exists an unlimited number of angular combinations having a zero projection. On the other hand most of those combinations are not physical and the true angular distribution depends mainly on three parameters: the exchange coupling at the interface $\left(J_{I}\right)$, which defines the 'starting' angle for the distribution with respect to the Fe interface; the exchange coupling in the GdN itself $\left(J_{G d N}\right)$, defining the angular increment between the magnetizations in the atomic planes and the magnitude of the magnetization of the planes that determines the Zeeman energy in the applied field. Therefore, the true distribution is not accessible through this experiment, since the integral projected net moment can be represented by different contributions that belong to different ratios of $J_{I}$ and $J_{G d N}$.

\subsubsection{Dichroism of the Fe L-edges}

All the experimental results discussed up to this point lead to the assumption that the magnetical twist in the GdN/Fe multilayers does not occur on a length scale predicted by Camley and others and the reason for this is obviously the much weaker coupling in GdN. Due to this weak coupling even at low temperatures and comparatively low fields we observe the 'modified' Fe aligned states that are located at high temperatures and higher fields in Camley's phase diagram. A direct proof of this state is based on the determination of the magnetic moment of the iron layers. In a Fe aligned state the projection of of the Fe moment onto the beam axis is $100 \%$ and therefore the expected magnetic moment should be close to the theoretical value of $2.2 \mu_{B}$ per atom. As discussed in chapter 3 the sum rules can be used to calculate the spin and orbital moment from the XMCD signal, even though they are not a universal tool. For the magnetic trasition metal elements like $\mathrm{Fe}$, Co and $\mathrm{Ni}$ the sum rules are widely confirmed and are used here to calculate the spin moment of the Fe layers. In Fig. 5.16 the absorption spectra $\mu^{+}$ and $\mu^{-}$(red and blue curve) of the $\mathrm{Fe} \mathrm{L}_{2,3}$-edge are shown. The spectra were measured in transmission on a $[\mathrm{GdN30/Fe25]x8} \mathrm{sample} \mathrm{on} \mathrm{mylar} \mathrm{in} \mathrm{an}$ 


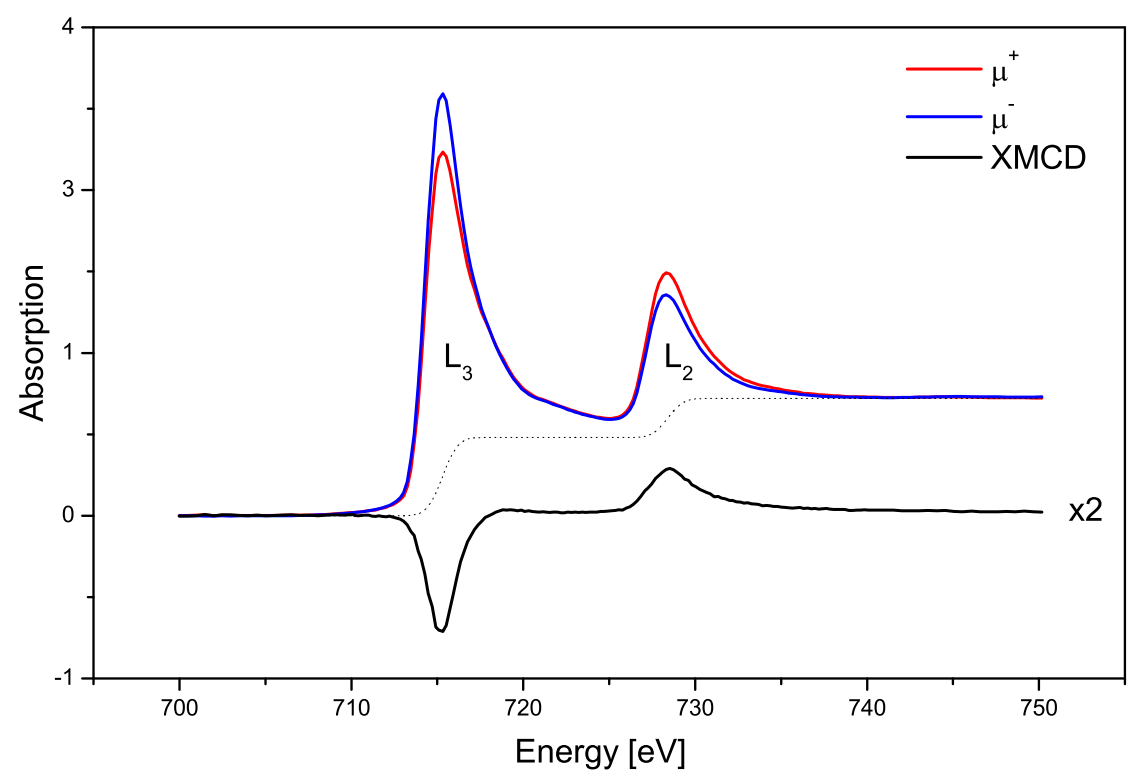

Figure 5.16: XAS absorption spectra $\mu^{+}$and $\mu^{-}$of the Fe $\mathrm{L}_{2,3}$-edges. The XMCD $\left(\mu^{+}-\mu^{-}\right)$is scaled by a factor of two

external field of $\pm 1 \mathrm{~T}$. To apply the sum rules the absorption spectra must be normalized and corrected for the edge jump. The step function representing the edge jump is set to $2 / 3(1 / 3)$ for the $\mathrm{L}_{3}\left(\mathrm{~L}_{2}\right)$ according to the degenerancy of the core states and normalized to 1 in the post edge region $(E>740 \mathrm{eV})$. The values for the spin and orbital moment are corrected for the projection angle and the degree of polarization of the beamline. At room temperature the sum rules give $2.0 \mu_{B}$ for the spin moment and $0.14 \mu_{B}$ for the orbital moment using a electron occupation number of 6.61 for iron [19]. The resulting total magnetic moment for Fe is then $2.14 \mu_{B}$ in good agreement with the theoretical value of $2.2 \mu_{B}$. This result validates the conclusions from the macroscopic magnetic measurements assuming that the Fe layers have the nominally magnetization of $1707 \mathrm{emu} / \mathrm{cm}^{3}$. Despite the careful treatment of the spectra taking into account uncertainties like beamline polarization a total error of $10 \%$ should be implied. In Fig. 5.17 the temperature dependence of the magnetic moment of the iron layers is shown. Obviously it varies only little with temperature. Especially in the region between $10 \mathrm{~K}$ and $80 \mathrm{~K}$ where the Gd L-edges show a drastic change with temperature, at the Fe L-edges only a slight decrease can be seen as it was indicated by the Fe K-edge results 


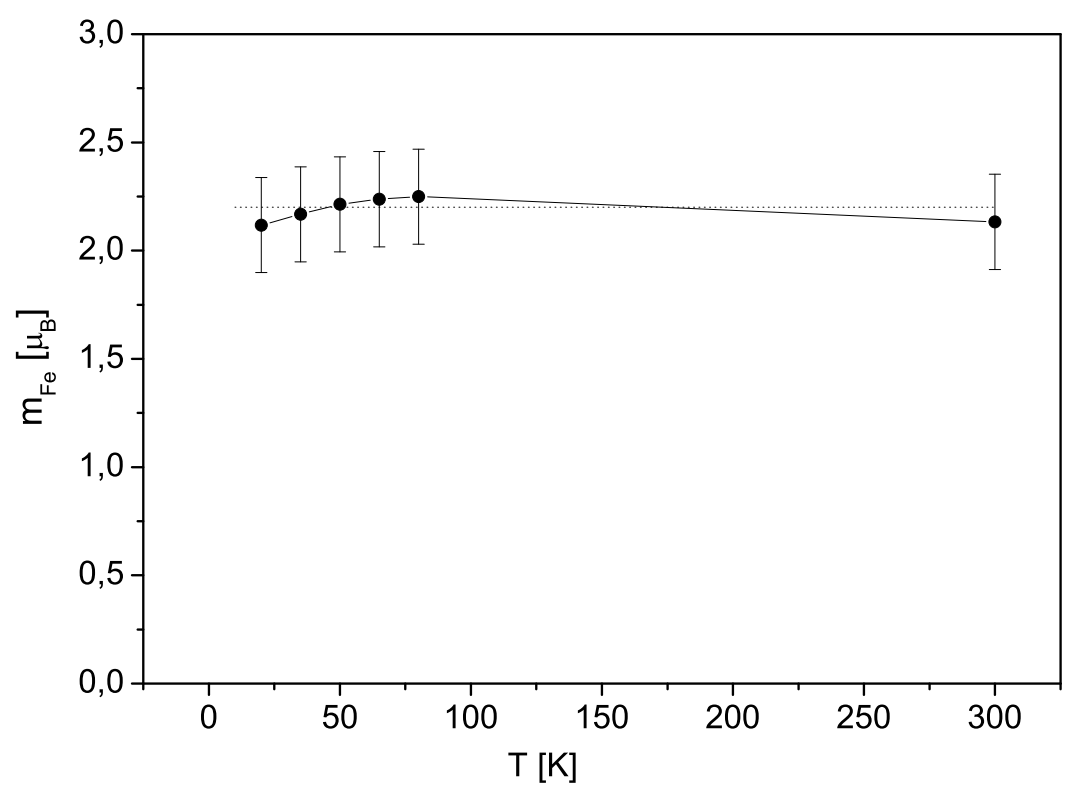

Figure 5.17: Temperature dependence of the Fe magnetic moment of a [Fe25/GdN30]x8 multilayer. The iron layers have a magnetic moment of $2.14 \mu_{B}$ /atom that varies only little with temperature.

in Fig. 5.13. When the temperature crosses the $T_{C}$ of the GdN one would expect a more drastic change in the projected magnetic moment of the iron since a twisted state should occur especially when reaching the compensation point of the sample. It is quite obvious that the iron layers do not form a twisted state and the slight decrease of the projected moment below the $\mathrm{T}_{C}$ of GdN can be interpreted as a small angular displacement of the Fe layer moments due to the increase of the torque caused by the antiparallel GdN. From the model of domain wall thickness where a $180^{\circ}$ twist in the Fe would take place over at least 62 monolayers [61] one can assume that the iron layer can be treated as a ferromagnetically aligned block with no twist of the magnetization between the atomic planes.

\subsubsection{Dichroism of the Gd M-edges}

With the Gd M-edges one probes the spin and orbital moment of the Gd If electrons that are connected via a strong parallel intraatomic spin-spin coupling to the $5 \mathrm{~d}$. Therefore the $4 \mathrm{f}$ magnetization should have the same 
temperature dependence as the $5 \mathrm{~d}$. The XMCD experiments concerning the Gd M-edges were all made at the beamline SU22 at LURE using the same set of samples that was used for the Fe L-edges. In contrast to the Fe Ledges the Rare Earth M-edges are not covered well by the sum rules, because in case of localized $4 \mathrm{f}$ electrons (like in Gd) there are also 4f-5d exchange interactions and the matrix elements are not necessarily equal for both spin channels (see chapter 3). Additionally, due to the small amount of material and the much smaller edge jump at the M-edges the determination of the magnetic moment of the $4 \mathrm{f}$ is not very reliable. Therefore the M-edges were normalized to the maximum amplitude of the isotropic $\mathrm{M}_{5}$ to refer them to the probed volume. However, the measured XMCD signal for a single GdN film at low temperatures gives a magnetic dichroism of about $20 \%$, which is in good agreement with other measurements on bulk Gd at low temperatures made at this beamline [76]. The general measurement was done in the same way as for the samples on Kapton. In Fig. 5.18 the absorption spectra

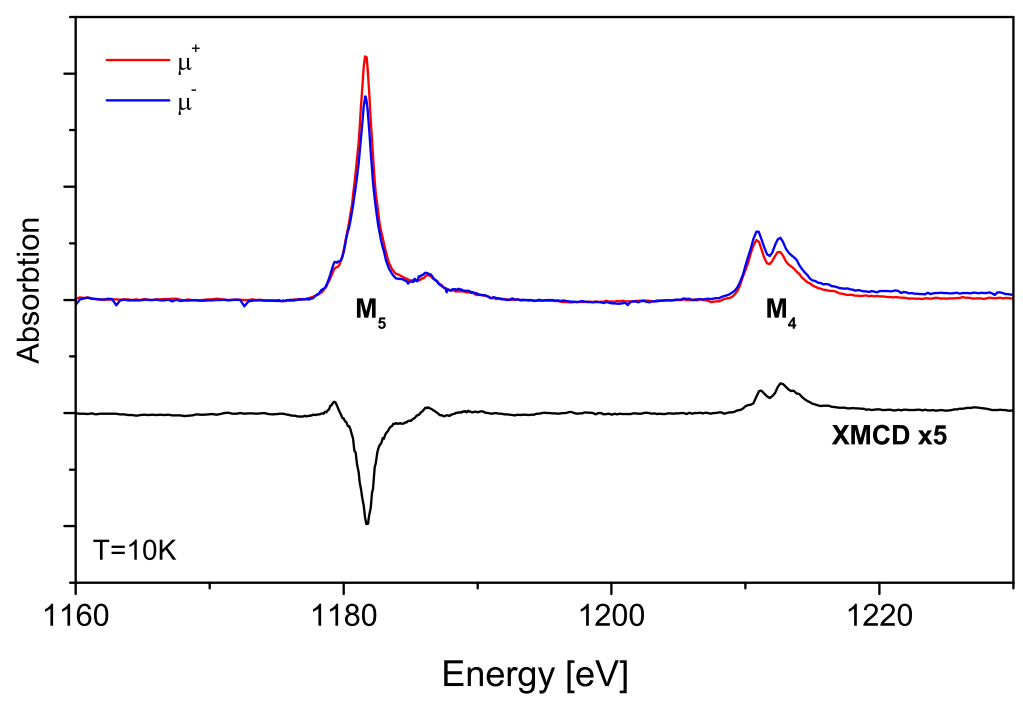

Figure 5.18: Absorption spectra $\mu^{+}$and $\mu^{-}$at the $\operatorname{Gd~} \mathrm{M}_{4,5}$-edges of the [GdN30/Fe25]x8 sample at $\mathrm{T}=10 \mathrm{~K}$. The XMCD (black curve) is scaled by a factor of 5

$\left(\mu^{+}\right.$and $\left.\mu^{-}\right)$for both magnetic field directions are shown. The black curve represents the XMCD $\left(\mu^{+}-\mu^{-}\right)$scaled by a factor of 5 for clarification. 


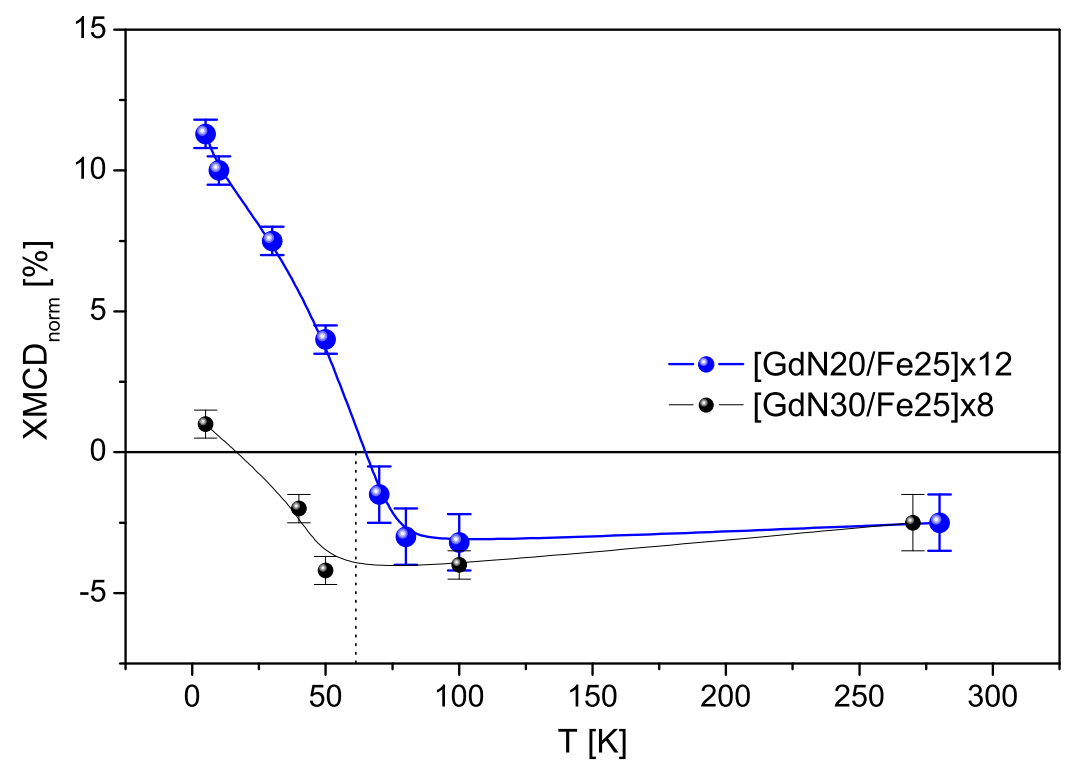

Figure 5.19: Temperature dependence of the normalize XMCD of the $\mathrm{Gd}_{4,5^{-}}$ edges. The run of the curves verify in good agreement the measurements at the Gd $\mathrm{L}_{2,3}$-edges

In Fig. 5.19 the temperature dependence of the Gd M-edge dichroism in an external magnetic field of $2 \mathrm{~T}$ is shown for [GdN20/Fe25]x12 (black) and [GdN30/Fe25]x8 (blue). Since the quality of the spectra is not as good as the Gd L-edge spectra the absolute error is larger, especially in the temperature region above $T_{C, G d N}$, when only a small amount of the GdN is polarized by the iron interface. At this point the advantage of the samples with a larger number of bilayers used at the Gd L-edges becomes obvious. But the trend of the curves is in good agreement with those determined from the Gd L-edges (Fig. 5.14). At room temperature one observes the same small negative polarization, that increases with decreasing temperature. Also in the region below $T_{C, G d N}$ the same drastic increase of the magnetic moment accompanied by a reversal of sign occurs. occurs. Even if the effect is not as clear as in the L-edge results, the sample with the $20 \AA$ GdN layer shows also a larger negative increase of the signal at lower temperatures and a nearly vanishing signal at $\mathrm{T}=10 \mathrm{~K}$, and one can conclude that the magnetic profile in these 
samples is the same as for those on kapton. This is not only a confirmation that the multilayers can be grown independently of the substrate even with a small number of bilayers. The twisted state in Camley's model is expanded over the whole supperlattice and the individual Fe and GdN layers are not twisted inside. Therefore the length over which a $360^{\circ}$ twist occurs is several bilayers. Here one observes no difference in the temperature dependence between a sample having 8 bilayers and one having 72 bilayers. The obviously easiest explanation is that a GdN/Fe supperlattice does not form such twisted state in external fields above 10kOe. Due to the weaker coupling in the GdN it is always a preferable situation to twist the GdN magnetization over a very short length scale (down to $20 \AA$ ) leaving the Fe layers magnetization mainly in the field direction.

\subsection{Elemental resolved Hysteresis}

A more direct evidence of the twist inside the GdN layers is an elemental resolved hysteresis and again the XMCD is a powerful tool to provide this type of experiment. In contrast to the usual XMCD measurements where the magnetic field is flipped to change between absorption of the majority and the minority holes for a elemental resolved hysteresis the helicity of the circular polarized x-ray has to be changed to gain the magnetic field as a free parameter. Like the remanent Gd L-edge measurements the elemental hysteresis was measured at the beamline ID24 at ESRF. In this case the Gd $\mathrm{L}_{2}$ edge was chosen to sense the magnetization in the GdN layers, which is far away from Fe K-edge EXAFS oscillations that are close to the $\mathrm{L}_{3}$-edge of $\mathrm{Gd}$ (see Fig. 5.12). This decision was based on the fact that the here used QWP method [77] suffers from a non-magnetic perturbation of the background that is caused by the diamond quarter-wave plate. Additionally K-edge EXAFS oscillation would complicate the situation needlessly. In this case the attempt to measure the Fe K-edge at the same time was not recommendable since the K-edge XMCD is small compared to the perturbations and the sampling time would be unmanageable long to achieve a usable XMCD. In Fig. 5.20 the hysteresis loops at the $\mathrm{Gd} \mathrm{L}_{2}$-edge (blue curves) and of the macroscopic magnetization for different temperatures of the [GdN50/Fe25]x52 sample are 


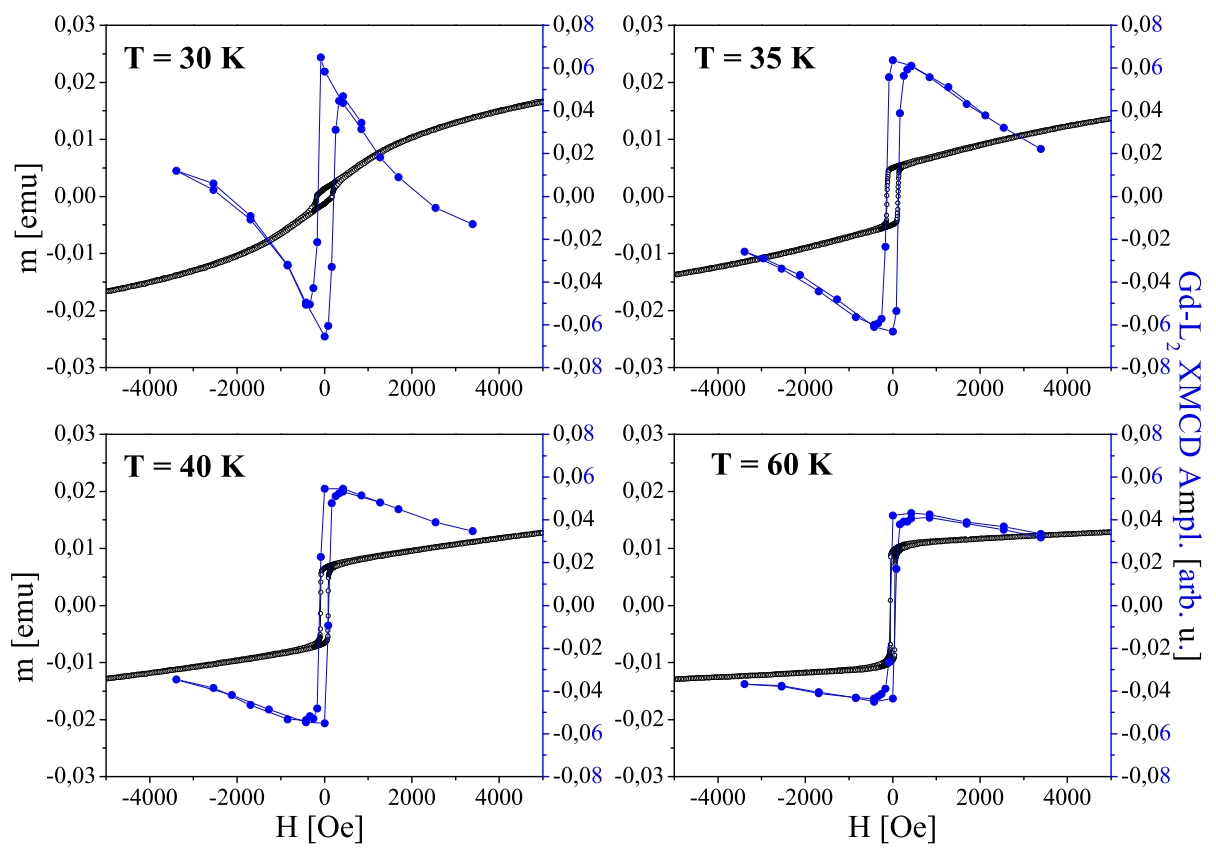

Figure 5.20: Macroscopic hysteresis (black) and $\mathrm{Gd} \mathrm{L}_{2}$ hysteresis (blue) for different sample temperatures

shown. For temperatures above the compensation point the $\mathrm{Fe}$ is aligned parallel to the field and the GdN antiparallel. The antiparallel alignment of the GdN can be seen from the positive $\mathrm{L}_{2}$ XMCD. For GdN aligned parallel to the magnetic field one would expect a positive $\mathrm{L}_{3}$ and a negative $\mathrm{L}_{2}$ signal like in figure 4.11 in chapter 4.5 . For temperatures near $T_{C, G d N}$ one finds a XMCD hysteresis curve with a $H_{C}=50$ Oe and a small negative slope with increasing field. The switching at $H_{C}$ reflects the reversal of the samples magnetization, that is all individual layers reverse their magnetization at the same time. A further increase of the field causes a stronger torque to the antiparallel aligned GdN magnetization due to the increasing Zeeman energy and the GdN layer magnetization are twisted slightly into the direction of the applied field leading to a decrease of the projected moment. Remembering the temperature dependence of the $\mathrm{L}_{3}$-edge XMCD in Fig. 5.14 in a external magnetic field of $20 \mathrm{kOe}$ one finds a significant change of the magnetization just below $60 \mathrm{~K}$. This is the onset of the rotation of th GdN layer magnetization. Above $60 \mathrm{~K}$ the magnetic moment of the GdN is low and the Zeeman 
energy is not large enough to induce a significant twist. Below 60K the magnetic moment of the GdN layers grows rapidly and increasing the field now twists the GdN layer due to the torque cause by the much larger Zeeman energy toward the field direction. As one can clearly see from the four hysteresis curves in Fig. 5.20 the negative slope of the $\mathrm{L}_{2}$-edge XMCD increases noticeable at lower temperatures. Due to some technical problems with the cryostat the temperature could not be lowered below $30 \mathrm{~K}$; the compensation point of the sample was just not reached. However, at $30 \mathrm{~K}$ one can clearly observe a change in the sign of the $\mathrm{Gd}_{2}$ hysteresis loop at an external field of $3 \mathrm{kOe}$. At this point the twist between the magnetizations in the atomic planes of the GdN has reached a net projection that is directed parallel to the field. Compared to the macroscopic hysteresis curves one observes only an increase of the magnetic moment with the external field. Although it was not possible to measure the Fe K-edge XMCD hysteresis to provide information about the alignment of the Fe layer magnetization one can conclude from the monotonic increase of the macroscopic hysteresis curves that there is almost no twist in the Fe layers.

Summing up the results from the macroscopic and the XMCD measurements the most obvious difference to Camley's model is that even at low temperatures the GdN layers are twisted inside by an external field. Recently Haskel et al. [54] investigated a Gd/Fe structure of comparable dimensions with elemental resolved XMCD hysteresis and compared it to theoretical results that are in agreement with the model of Camley. For temperatures well above (or below) the compensation point they find only a Fe (or Gd) aligned phase, that are always antiferromagnetically coupled. In the region of the compensation point they observe, depending on temperature, either a surface twisted or a bulk twisted phase. At $10 \mathrm{~K}$ their result shows that in a $\mathrm{Gd} / \mathrm{Fe}$ layer a twist would be to costly in exchange energy and therefore the $\mathrm{Gd} / \mathrm{Fe}$ system prefers the Gd aligned phase. The GdN/Fe system examined here can form a "GdN twisted only" phase GdN* even at low temperature due to the much smaller exchange in the GdN. This is similar to the 'modified' Fe aligned phases in Camley's diagram (Fig. 5.2) labeled Gd* but in contrast to his model in this system the modified phases are at lower temperatures and extended over larger range. To draw a detailed phase diagram more 


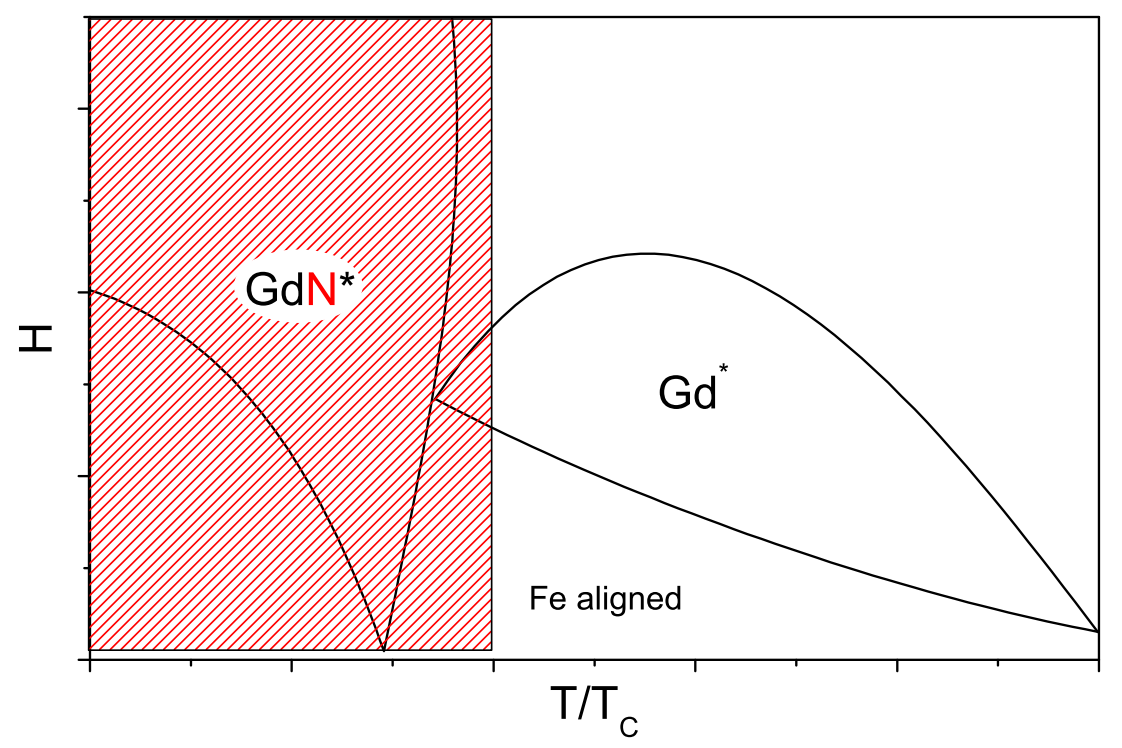

Figure 5.21: Camley's phase diagram based on a Gd/Fe system. The red hatched area marks the estimated GdN twisted phase that occurs even at low fields and low temperatures due to the much smaller exchange coupling in the GdN.

data would be necessary, especially detailed $M(H)$ curves for the Fe and the GdN layers. From the results made here one can mark roughly the area in Camley's phase diagram were the GdN* phase would occur. The immediate onset of negative slope in the $\mathrm{L}_{2}$-edge XMCD hysteresis at low temperature indicates that the Gd aligned phase exists in the limit to low fields only and external fields in the range of several hundreds of Oe can twist the GdN magnetization inside well below the compensation point. Above $T_{C, G d N}$ the GdN twisted phase disappears since the field cannot induce a twist due to the lower Zeeman Energy. An estimated region of the GdN twisted phase in Camley's phase diagram is drawn in Fig. 5.21.

At a first glance the extreme sensitivity to an external field points to a very low exchange coupling in the GdN. But an estimation using Motokawa's model leads to a much to small exchange constant as one would expect from the $T_{C}$ of GdN within the molecular field theory. Furthermore the enhancement of the magnetization in the GdN layer with decreasing thickness (Fig. 5.7) is hardly to explain with such a low exchange interaction. The magnetic exchange in the GdN with strong localized $4 \mathrm{f}$ electrons with a large magnetic moment of $7 \mu_{B}$ that are weakly coupled through a RKKY exchange via the 
$5 \mathrm{~d}$ electrons has obviously interesting consequences in a superlattice. The weak coupling of the $5 \mathrm{~d}$ lead to a low $T_{C}$ of $59 \mathrm{~K}$ but the presence of the iron can enhance the coupling over a large temperature range as proposed by Wang and Mills. On the other hand a small external field breaks up the antiferromagnetic alignment to the Fe and causes a twist inside the GdN over a very short length scale and a critical field as in Motokawa's model was not clearly observed here.

\subsection{Summary}

It could be shown that $\mathrm{GdN} / \mathrm{Fe}$ multilayers can be grown at room temperature with a (200) texture with sharp interfaces. The GdN layers are antiferromagnetically coupled to the iron layers as expected from the model of Johansson [49]. From the Gd L-edge XMCD one observes a polarization of the GdN induced by the iron well above $T_{C, G d N}$ on a length scale of $5 \AA$. The temperature dependence of the GdN magnetization in the multilayers is different from the observed one in bulk GdN. Due to the small layer thicknesses the multilayers are not describable by a superposition of the bulk materials, but behave more as a coherent structure as proposed by Wang and Mills [75]. The GdN lattice shows a distortion at the interface to the Fe and the reduced in-plane next-neighbor spacing of the GdN enhances the magnetic coupling in the GdN layers. Together with the influence of the Fe polarization in the frame of the model of Wang and Mills one observes a distinctly increased magnetization above the bulk Curie temperature of GdN. On the other hand an external magnetic field can twist the GdN magnetization in the atomic layers easily and break up the antiferromagnetic alignment of the multilayers. Even at low temperatures a small magnetic field causes a rotation in the GdN layers, which is demonstrated by the elemental resolved XMCD hysteresis loops at the Gd L-edges. 


\section{Chapter 6}

\section{Summary}

In the frame of this work the ferromagnetic semiconductor GdN and GdN/Fe multilayers were examined with XMCD spectroscopy. The elemental resolved observation of the polarization of the valence states in GdN and GdN/Fe multilayers provides valuable information about the magnetic coupling in GdN and GdN/Fe multilayers. In GdN the magnetic polarization of the Gd 4f, Gd 5d and N 2p states can be shown. The temperature dependence of the $\mathrm{XMCD}$ compared to the macroscopic magnetization confirms the assumption that the $4 \mathrm{f}$ moments of the Gd ions are coupled via a polarization of the Gd $5 \mathrm{~d}$ and the $\mathrm{N} 2 \mathrm{p}$ electron states.

In the first part of this thesis GdN thin films were investigated and it was found that it is possible to grow polycrystalline GdN thin films in a rocksalt structure $(\mathrm{NaCl})$ on various substrates. The macroscopic magnetic properties of the GdN films are the same as in bulk GdN. The semiconducting nature of the GdN films could be shown by resistivity measurements and one observes a change to a metal-like conduction below $T_{C}$ as in EuO. The magnetic coupling in GdN is related to a ferromagnetic RKKY exchange coupling of the $\mathrm{Gd} 5 \mathrm{~d}$ electrons. Their polarization is clearly visible in the $\mathrm{Gd} \mathrm{L}-$ edge XMCD and the temperature dependence of the Gd L-edges is the same as for the macroscopic magnetization, stating the connection to the Gd $4 \mathrm{f}$ moment. The $\mathrm{N} 2 \mathrm{p}$ electron states are also involved in the magnetic coupling and one can observe their polarization in a XMCD signal at th N K-edge. In agreement with theory and the magnetic measurements made here one can 
conclude that the $\mathrm{N} 2 \mathrm{p}$ electrons are antiferromagnetically coupled to the Gd $5 \mathrm{~d}$ electrons and the moments compensate each other. The expected redshift for GdN below $T_{C}$ is visible in a small shift of the x-ray absorption onset of the $\mathrm{Gd} \mathrm{L}_{2}$-edge.

In the second part the magnetic properties of $\mathrm{GdN} / \mathrm{Fe}$ multilayers are examined and compared to the well know Gd/Fe system. The GdN/Fe multilayers can be grown at room temperature with sharp interfaces. Due to the polarization of the iron and the small layers thicknesses the GdN layers show an enhanced magnetization above $T_{C, G d N}$ that might be additionally heightened by a tetragonal distortion of the GdN lattice at the iron interface. But in contrast to the $\mathrm{Gd} / \mathrm{Fe}$ system the magnetization in the GdN atomic layers is easily twisted by an external magnetic field. In $\mathrm{Gd} / \mathrm{Fe}$ multilayers one would expect no twist inside the Gd at low temperatures and low fields. In the $\mathrm{GdN} / \mathrm{Fe}$ multilayers the GdN is already twisted at low temperatures by an external field of about 5000e. 


\section{Bibliography}

[1] G. Schmidt, D. Ferrand, L. W. Molenkamp, A. T. Filip, and B. J. van Wees, Physical Review B 62, 4790 (2000).

[2] A. T. Filip, P. LeClair, C. Smits, J. T. Kohlhepp, H. Swagten, B. Koopmans, and W. de Jonge, Applied Physics Letters 81, 1815 (2002).

[3] P. LeClair, J. K. Ha, H. J. M. Swagten, J. T. Kohlhepp, C. H. van de Vin, and W. J. M. de Jonge, Applied Physics Letters 80, 625 (2002).

[4] N. Samarth, S. Chun, K. Ku, S. Potashnik, and P. Schiffer, Solid State Communications 127, 173 (2003).

[5] N. Teraguchi, A. Suzuki, Y. Nanishi, Y.-K. Zhou, M. Hashimoto, and H. Asahi, Solid State Communications 122, 651 (2002).

[6] B. D. Cullity, Elements of x-ray diffraction, 2nd ed. (Addison-Wesely, United States of America, 1978).

[7] L. G. Paratt, Physical Review 95, 359 (1954).

[8] V. O. de Haan and G. G. Drijkoningen, Physica B 198, 24 (1994).

[9] WIN-REFSIM, Bruker AXS GmbH Version 2.0.

[10] Parratt reflectivity tool by Chritian Braun, HMI Berlin.

[11] G. Schatz and A. Weidinger, Nukleare Festkörperphysik, 3 ed. (Teubner Studienbücher, Stuttgart, 1997).

[12] H. Ebert, J. Stöhr, S. S. P. Parkin, M. Samant, and A. Nilsson, Physical Review B 53, 16067 (1996). 
[13] J. L. Erskin and E. A. Stern, Physical Review B 12, 5016 (1975).

[14] G. Schütz, W. Wagner, W. Wilhelm, P. Kienle, R. Zeller, R. Frahm, and G. Materlik, Physical Review B 58, 737 (1987).

[15] B. T. Thole, P. Carra, F. Sette, and G. van der Laan, Physical Review Letters 68, 1943 (1992).

[16] P. Carra, B. T. Thole, M. Altarelli, and X. Wang, Physical Review Letters 70, 694 (1993).

[17] J. Stöhr, Journal of Magn. and Mag. Mat. 200, 470 (1999).

[18] M. Altarelli, Physical Review B 47, 597 (1993).

[19] C. T. Chen, Y. U. Idzerda, H.-J. Lin, N. V. Smith, G. Meigs, E. Chaban, G. H. Ho, E. Pellegrin, and F. Sette, Physical Review Letters 75, 152 (1995).

[20] B. N. Harmon and A. J. Freeman, Physical Review B 10, 1979 (1974).

[21] J. C. Lang, X. Wang, V. P. Antropov, B. N. Harmon, , A. I. Goldman, H. Wan, G. C. Hadjipanayis, and K. D. Finkelstein, Physical Review B 49, 5993 (1994).

[22] F. Baudelet, C. Giorgetti, S. Pizzini, C. Brouder, D. Dartyge, A. Fontaine, J. Kappler, and G. Krill, Journal of Electron Spectroscopy related Phenomena 62, 153 (1993).

[23] J. Stoehr and Y. Wu, in New directions in research with third-generation soft x-ray synchrotron radiation sources, edited by A. S. Schlachter and F. J. Wuilleumier (Kluwer Academic Publishers, Netherlands, 1994).

[24] http://www.esrf.fr/exp_facilities/ID24/ID24.html

[25] P. Wachter, in Handbook of Physics and Chemistry of Rare Earth, edited by K. A. Gschneider and L. Eyring (North-Holland, Amsterdam, 1979), Vol. 2.

[26] Y. Shapira, S. Foner, and T. B. Reed, Physical Review B 8, 2299 (1973). 
[27] P. Wachter and E. Kaldis, Solid State Communications 34, 241 (1980).

[28] D. X. Li, Y. Haga, H. Shida, and T. Suzuki, Physica B 199\&200, 631 (1994).

[29] D. Li, Y. Haga, H. Shida, T. Suzuki, Y. Kwon, and G. Kido, Journal of Physics: Condensed Matter 9, 10777 (1997).

[30] T. Kasuya and D. X. Li, Journal of Magnetism and Magnetic Materials 167, L1 (1997).

[31] T. Kasuya and D. X. Li, Physica B 230-232, 472 (1997).

[32] A. Hasegawa and A. Yanase, J. Phys. Soc. Japan 42, 492 (1976).

[33] S. Methfessel, Zeitschrift für Angewandte Physik 18, 414 (1965).

[34] T. R. McGuire, B. E. Argyle, M. W. Shafer, and J. S. Smart, Journal of Applied Physics 34, 1345 (1963).

[35] W. Lambrecht, Physical Review B 62, 13538 (2000).

[36] C. M. Aerts, P. Strange, M. Horne, W. M. Temmerman, Z. Szotek, and A. Svane, Physical Review B 69, 045115 (2004).

[37] H. Holleck, E. Smailos, and F. Thümmler, Journal of Nuclear Materials 32, 289 (1969).

[38] A. Parge, Diplomarbeit, Universiät Göttingen, 2003.

[39] A. Arrott, Physical Review 108, 1394 (1957).

[40] A. Arrott and J. E. Noakes, Physical Review Letters 19, 786 (1967).

[41] A. G. Petukhov, W. R. L. Lambrecht, and B. Segall, Physical Review B 53, 4324 (1996).

[42] P. G. Steeneken, L. H. Tjeng, I. Elfimov, G. A. Sawatzky, G. Ghiringhelli, N. B. Brookes, and D.-J. Huang, Physical Review Letter 88, 47201 (2002). 
[43] A. Urushibara, Y. Moritomo, T. Arima, A. Asamitsu, G. Kido, and Y. Tokura, Physical Review B 51, 14103 (1995).

[44] M. van Veenendaal, J. B. Goedkoop, , and B. T. Thole, Physical Review Letters 78, 1162 (1997).

[45] M. Imada, A. Fujimori, and Y. Tokura, Review of Modern Physics 70, 1039 (1998).

[46] D. J. Khomskii and G. A. Sawatzky, Solid State Communications 120, 87 (1997).

[47] A. Perlov (unpublished).

[48] E. Goering, A. Fuss, W. W. J. Will, and G. Schütz, Journal of Applied Physics 88, 5920 (2000).

[49] B. Johansson, L. Nordström, O. Eriksson, and M. S. S. Brooks, Physica Scripta T39, 100 (1991).

[50] M. Brooks, L. Nordström, and B. Johansson, Physica B 172, 95 (1991).

[51] M. Brooks and B. Johansson, in Handbook of magnetic materials, edited by K. Buschow (North-Holland, Amsterdam, 1993), Vol. 7.

[52] J. L. Prieto, B. B. van Anken, G. Burnell, C. Bell, J. E. Evetts, N. Mathur, and M. G. Blamire, Physical Review B 69, 054436 (2004).

[53] A. P. Popov and A. V. Anisimov, Physical Review B 67, 094428 (2003).

[54] D. Haskel, G. Srajer, Y. Choi, R. D. Lee, J. C. Lang, J. Meerschaut, J. S. Liang, and S. D. Bader, Physical Review B 67, 180406 (2003).

[55] D. Haskel, G. S. nad J. C. Lang, J. Pollmann, C. S. Nelson, J. Jiang, and S. D. Bader, Physical Review Letters 87, 207201 (2001).

[56] R. E. Camley, Physical Review B 39, 12316 (1989).

[57] R. E. Camley, Physical Review B 35, 3608 (1987).

[58] L. L. Hinchey and D. Mills, Physical Review B 33, 3329 (1986). 
[59] K. Cherifi, C. Dufour, P. Bauer, G. Marchal, and P. Mangin, Physical Review B 44, 7733 (1991).

[60] J. G. LePage and R. E. Camley, Physical Review Letters 65, 1152 (1990).

[61] C. Kittel, Einführung in die Festkörperphysik, 10 ed. (Oldenbourg, München, 1993).

[62] M. Motokawa and H. Dohnomae, Journal of The Physical Society of Japan 60, 1355 (1991).

[63] M. Motokawa, Progress of Theoretical Physics Supplement 101, 537 (1990).

[64] K. Takanashi, Y. Kamiguchi, H. Fujimori, and M. Motokawa, Journal of The Physical Society of Japan 61, 3721 (1992).

[65] W. Hahn, M. Loewenhaupt, Y. Huang, G. Felcher, and S. Parkin, Physical Review B 52, 16041 (1995).

[66] A. Kozumi, M. Takagaki, M. Suzuki, N. Kawamura, and N. Sakai, Physical Review B 61, 14909 (2000).

[67] O. F. K. McGrath, N. Ryzhanova, C. Lacroix, D. Givord, C. Ferman, C. Miramond, G. Saux, S. Young, and A. Vedyayev, Physical Review B 54, 6088 (1996).

[68] M. Sajieddine, P. Bauer, K. Cherifi, C. Dufour, and G. Marchal, Physical Review B 49, 8815 (1994).

[69] M. Münzenberg, Ph.D. thesis, Universität Göttingen, 2000.

[70] W. Lohstroh, Ph.D. thesis, Universität Göttingen, 1999.

[71] M. Arend, Ph.D. thesis, Universität Göttingen, 1998.

[72] M. Farle, K. Baberschke, U. Stetter, A. Aspelmeier, and F. Gerhardter, Physical Review B 47, 11571 (1993). 
[73] P. Srivastava, F. Wilhelm, A. Ney, M. Farle, H. Wende, N. Haack, G. Ceballos, and K. Baberschke, Physical Review B 58, 5701 (1998).

[74] P. Jensen, K. H. Bennemann, P. Poulopoulos, M. Farle, F. Wilhelm, and K. Baberschke, Physical Review B 60, 14994 (1999).

[75] R. Wang and D. L. Mills, Physical Review B 46, 11681 (1992).

[76] J.-P. Kappler, private communications.

[77] S. Pizzini, M. Bonfim, F. Baudelet, H. Tolentino, A. S. Miguel, K. Mackay, C. Malgrange, M. Hagelstein, and A. Fontaine, J. Synchrotron Rad. 5, 1298 (1998). 


\section{Acknowledgment}

This thesis is the result of four and half years of work whereby I have been accompanied and supported by many people. I have now the opportunity to express my gratitude to all of them.

- First of all I would like to thank Wolfgang Felsch as our maître cuisinier for providing me the possibility to brew this work and for his confidence in me. He was never tired discussing with me about this work and shared his great experience all the way to wherever we went. The environment he creates in our group is fruitful and carefree and helped me every day to concentrate on my work with the necessary joy and care. Last but not least he was my tour guide in France, a land of confusion to me. My French will be better in my next life... promised.

- Kai Fauth and Markus Heßler for their all-time help at the beamline PM3 at BESSY making the beautiful N K-edge measurements possible and for the discovery of "Ecki's Döner Bistro" in Berlin.

- Françios Baudelet, Christine Giorgetti, Gérard Krill, Franck Fortuna and Phillip Ohresser for their help and their inspiring discussions during our beamtimes at LURE.

- Thomas Neisius and Olivier Mathon for providing every assistance and beamline tweaking at ESRF

- Coryn Hague and his group for measuring the total fluorescence yield "along the way" during their own beamtime.

- Wiebke Lohstroh and Markus Arend for taking in a blissful greenhorn into a world where physics becomes touchable.

- Anne Parge for surviving all our beamtimes where the diurnal rhythm was like a "staccato scherzo" and for her merciless listening to whatever I was talking about.

- Björn Sass for being the "aurora borealis" in our group with down-toearth problem solving capabilities and being my personal firefighter. Alles im Lot auf'm Boot... 
- Christian Tusche for his helpful discussions, the cool lab updates and his matchless skill in wrapping things into tinfoil.

- Markus Münzenberg for being my friend all the time sharing many funny times and frequent cycling breaks with me. Don't forget, we have to settle old scores with "Die Gleichen".

- Henning Harms (the real king of barbecue) for irradiating me every day with "good vibrations" and for a curly kale session with the real taste of the "Lüneburger Heide".

- Klaus Krug for being a paradigm in all matters about meat and the insight that a high grade meal must contain at least three different kinds of roasted meat.

- Andrea Käufler for her multidimensional power of imagination and her adjusted concepts in German grammar.

- Fabian Giesen for the all the discussions about us and our world in context with Captain Future in speakeasies where the future is past.

- Uta Bete and Carsten Mahn for being the fairy godmothers in our lab and for their patience with laborious scientists.

- Everybody in our mechanical workshop for the great help and for drilling everything "from scratch" and explaining me with anxious gazes why I should not do-that-myself.

- Everybody in the Institute for the comfy ambiance, all the discussions, the barbecues, the soccer matches and all the other things I cannot mention here but I will never forget.

Last but not least I would like to thank my brother and my parents for all the support in my life and all the invaluable moments.

That's all folks. Thanks a lot... 


\title{
Lebenslauf
}

\author{
Name: $\quad$ Frank Leuenberger \\ Geburtstag: $\quad 22.04 .1968$ \\ Geburtsort: Bern (Schweiz) \\ Staatsangehörigkeit: deutsch
}

Schulausbildung:

1975-1977: Grundschule Kanton Bern

1977-1979: Gorch-Fock-Schule Cuxhaven

1979-1989: Amandus-Abendroth-Gymnasium Cuxhaven

Zivildienst:

1989-1990: Lebenshilfe e.V. Cuxhaven

Hochschulausbildung:

Studium der Physik an der Universität Göttingen vom

WS 1991/1992 - SS 2004

1994: $\quad$ Vordiplom

1999: Diplom, Thema der Diplomarbeit:

Untersuchungen zur Schichtdickenabhängigkeit

des Metall-Halbleiter-Übergangs

in Lanthanhydrid-Schichten

2000-2004: Wissenschaftlicher Angestellter im Sonderforschungsbereich 345/602 der Universität Göttingen

Anfertigen der vorliegenden Dissertation am

I. Physikalischen Institut 